\title{
Bihamiltonian Hierarchies in 2D Topological Field Theory At One-Loop Approximation
}

\author{
Boris Dubrovin* Youjin Zhang** \\ * SISSA, Via Beirut 2-4, 34014 Trieste, Italy \\ email: dubrovin@sissa.it \\ ** Division of Mathematics, Graduate School of Science \\ Kyoto University, Kyoto 606-8502, Japan \\ email: youjin@kusm.kyoto-u.ac.jp
}

\begin{abstract}
We compute the genus one correction to the integrable hierarchy describing coupling to gravity of a 2D topological field theory. The bihamiltonian structure of the hierarchy is given by a classical $W$-algebra; we compute the central charge of this algebra. We also express the generating function of elliptic Gromov - Witten invariants via tau-function of the isomonodromy deformation problem arising in the theory of WDVV equations of associativity.
\end{abstract}




\section{Introduction}

According to [5, 8, 31, the primary free energy of the matter sector of a 2D topological field theory (TFT) with $n$ primaries as a function $F(t)$ of the coupling constants $t=\left(t^{1}, \ldots, t^{n}\right)$ must satisfy WDVV equations of associativity. The problem of selection of physical solutions among all the solutions to WDVV equations is still open. Reformulating, the problem is to understand which part of the building of a $2 \mathrm{D}$ TFT can be constructed taking an arbitrary solution of WDVV equations as the basement.

The first problem to be settled is coupling of a given matter sector to topological gravity. In the full theory, besides the primaries $\phi_{1}=1, \phi_{2}, \ldots, \phi_{n}$ that we now redenote $\phi_{1,0}, \ldots, \phi_{n, 0}$, there are infinite number of their gravitational descendents $\phi_{1, p}, \ldots, \phi_{n, p}, p=1,2, \ldots$ The generating function of their correlators is the full free energy of the theory

$$
\mathcal{F}(T)=\left\langle e^{\sum T^{\alpha, p} \phi_{\alpha, p}}\right\rangle
$$

here $T^{\alpha, p}$ are the coupling constants correspondent to the fields $\phi_{\alpha, p}$,

$$
\langle\ldots\rangle:=\sum_{g \geq 0} \int_{\Sigma_{g}} \ldots e^{-S[\psi]}[d \psi]
$$

(the sum over the fields $\psi$ living on the surface of genus $g, S$ is the classical action). According to the idea of Witten [32] this procedure of coupling to topological gravity must be described by an integrable hierarchy of PDEs. The unknown functions of the hierarchy are the particular two-point correlators

$$
v_{\alpha}:=\left\langle\phi_{\alpha, 0} \phi_{1,0} e^{\sum T^{\beta, q} \phi_{\beta, q}}\right\rangle=\frac{\partial^{2} \mathcal{F}(T)}{\partial T^{\alpha, 0} \partial T^{1,0}},
$$

$T^{1, p}, \ldots, T^{n, p}$ are the times of the $p$-th flow of the hierarchy, and the cosmological constant $X:=T^{1,0}$ plays the role of the spatial variable of the hierarchy. The partition function of the full theory is the $\tau$-function of a particular symmetric solution of the hierarchy. This idea works perfectly well for the case of pure gravity (the matter sector is trivial, $\left.n=1, F(t)=\frac{1}{6} t^{3}\right)$. According to the theory of Witten - Kontsevich [26, 33] the partition function of $2 \mathrm{D}$ gravity is a particular $\tau$-function of the $\mathrm{KdV}$ hierarchy.

For a 2D TFT with a nontrivial matter sector the correspondent integrable hierarchies are not known, although there are interesting conjectures about their structure for topological minimal models [7], for $C P^{1}$ topological sigm-model [11, 14, 15, 16, 17]. However, the properties of genus expansion of a 2D TFT provide us with certain nontrivial assumptions about the structure of the hypothetical hierarchy. Denote $\mathcal{F}_{g}$ the genus $g$ part of the free energy

$$
\begin{gathered}
\mathcal{F}_{g}:=\left\langle e^{\sum T^{\alpha, p} \phi_{\alpha, p}}\right\rangle_{g}, \\
\mathcal{F}=\sum_{g \geq 0} \mathcal{F}_{g} .
\end{gathered}
$$


Particularly, the primary free energy is obtained restricting $\mathcal{F}_{0}$ onto the small phase space $T^{\alpha, p>0}=0$

$$
F(t)=\left.\mathcal{F}_{0}\right|_{T^{\alpha, 0}=t^{\alpha}}, \quad T^{\alpha, p>0}=0 .
$$

The procedure of genus expansion consists of the following two parts.

1). We introduce slow spatial and time variables rescaling

$$
X \mapsto \varepsilon X, \quad T^{\alpha, p} \mapsto \varepsilon T^{\alpha, p} .
$$

2). We change

$$
\mathcal{F} \mapsto \sum_{g=0}^{\infty} \varepsilon^{2 g-2} \mathcal{F}_{g}
$$

The indeterminate $\varepsilon$ is called string coupling constant. As $\varepsilon \rightarrow 0$ one has a singular limit of the tau-function (i.e., of the partition function) of the theory

$$
\tau(T, \varepsilon):=\exp \left(\varepsilon^{-2} \mathcal{F}_{0}+\mathcal{F}_{1}+\varepsilon^{2} \mathcal{F}_{2}+\ldots\right) .
$$

Also all of the correlators become series in $\varepsilon$. Particularly, the series of the two-point correlators (1.2) have the form

$$
v_{\alpha}=\sum_{g=0}^{\infty} \varepsilon^{2 g} v_{\alpha}^{g}
$$

where

$$
v_{\alpha}^{g}:=\left\langle\phi_{\alpha, 0} \phi_{1,0} e^{\sum T^{\beta, q} \phi_{\beta, q}}\right\rangle_{g}=\frac{\partial^{2} \mathcal{F}_{g}}{\partial T^{\alpha, 0} \partial X} .
$$

The genus zero (i.e., the tree-level) approximation of the theory corresponds to the dispersionless approximation of the hierarchy. The solution $\left(v_{1}^{0}, \ldots, v_{n}^{0}\right)$ to the hierarchy is given by the genus zero two-point correlators $v_{\alpha}^{0}=\left\langle\phi_{\alpha, 0} \phi_{1,0} e^{\sum T^{\beta, q} \phi_{\beta, q}}\right\rangle_{0}$ as the functions of the couplings. The solution is specified by the initial data on the small phase space

$$
\left.v_{\alpha}^{0}\right|_{T^{\alpha, p>0}=0}=\eta_{\alpha \beta} T^{\beta, 0}
$$

where the constant "metric" $\eta_{\alpha \beta}$ is specified by the primary correlators of the form

$$
\eta_{\alpha \beta}:=\left.\left\langle\phi_{1,0} \phi_{\alpha, 0} \phi_{\beta, 0}\right\rangle_{0}\right|_{T^{\alpha, p>0}=0} .
$$

The construction of the would-be dispersionless approximation of the unknown integrable hierarchy for an arbitrary solution to equations of associativity and of the needed $\tau$-function of it was given in [9] in terms of the geometry of WDVV equations (see also [1]). The bihamiltonian structure of the hierarchy was found in [10]. We briefly recollect this construction in Section 2 below. (We also describe more accurately the quasihomogeneity property of the hierarchy and of the $\tau$-function formulated in [9] only for a generic solution of WDVV equations).

One can try to go beyond the tree-level approximation expanding the unknown hierarchy in a series w.r.t. $\varepsilon^{2}$. The string coupling constant $\varepsilon$ plays the role of the dispersion parameter. The reader can keep in mind the dispersion expansion

$$
u_{t}=u u_{x}+\frac{1}{12} \varepsilon^{2} u_{x x x}
$$


of the KdV equation as an example of such a series. Particularly, for the one-loop (i.e., genus $=1$ ) approximation of the theory, it is sufficient to retain the terms of the hierarchy up to the $\varepsilon^{2}$ order. Particular solutions of the one-loop approximation must have the form

$$
\begin{aligned}
v_{\alpha} & =v_{\alpha}^{0}(T)+\varepsilon^{2} v_{\alpha}^{1}(T)+\mathcal{O}\left(\varepsilon^{4}\right) \\
& =\left\langle\phi_{\alpha, 0} \phi_{1,0} e^{\sum T^{\beta, q} \phi_{\beta, q}}\right\rangle_{0}+\varepsilon^{2}\left\langle\phi_{\alpha, 0} \phi_{1,0} e^{\sum T^{\beta, q} \phi_{\beta, q}}\right\rangle_{1}+\mathcal{O}\left(\varepsilon^{4}\right) \\
& =\frac{\partial^{2}}{\partial T^{\alpha, 0} \partial T^{1,0}}\left(\mathcal{F}_{0}(T)+\varepsilon^{2} \mathcal{F}_{1}(T)\right)+\mathcal{O}\left(\varepsilon^{4}\right)
\end{aligned}
$$

So for $\varepsilon=0$ the one-loop approximation becomes the already known tree-level approximation of the hierarchy. We will call the genus one approximation of the integrable hierarchy the one-loop deformation of the genus zero hierarchy.

Our result is that, under the assumption of semisimplicity (see below) the oneloop deformation of the hierarchy exists for any solution of WDVV equations and it is uniquely determined by the general properties of the genus one correlators proved by Dijkgraaf and Witten [6] and by Getzler [21]. (For the solution of WDVV equations with one and two primaries the one-loop approximation of the hierarchy was constructed in [6, 16]). Recall that the genus one part of the free energy has the form

$$
\mathcal{F}_{1}(T)=\left[\frac{1}{24} \log \operatorname{det} M_{\alpha \beta}\left(t, \partial_{X} t\right)+G(t)\right]_{t=v^{0}(T)},
$$

where the matrix $M_{\alpha \beta}$ has the form

$$
\begin{aligned}
& M_{\alpha \beta}\left(t, \partial_{X} t\right)=c_{\alpha \beta \gamma}(t) \partial_{X} t^{\gamma}, \\
& c_{\alpha \beta \gamma}(t)=\partial_{\alpha} \partial_{\beta} \partial_{\gamma} F(t),
\end{aligned}
$$

and $G(t)$ is a certain function specified by Getzler's equation [21] (see also Section 6 below). The first part of the formula becomes trivial on the small phase space $T^{\alpha, p}=0$ for $p>0$. The second part describes, in the topological sigma-models, the genus one Gromov-Witten invariants of the target space. For this function we derive the following formula

$$
G=\log \frac{\tau_{I}}{J^{1 / 24}}
$$

(as above, semisimplicity of the solution of WDVV is assumed). Here $J$ is the Jacobian of the transform between canonical and flat coordinates (see Sect. 2 below). To explain who is $\tau_{I}$ we recall that, in the semisimple case, WDVV can be reduced to equations of isomonodromy deformations of a certain linear differential operator with rational coefficients [9]. Our $\tau_{I}$ is the tau-function of the solution of these equations of isomonodromy deformations in the sense of [24]. According to [24], [30] the tau-function appears as the Fredholm determinant of an appropriate Riemann-Hilbert boundary value problem (see [12] for reduction of WDVV equations to a boundary value problem). Remarkably, the formula makes sense for an arbitrary semisimple solution of WDVV equations. Using explicit expressions (2.17), (2.19) for $\tau_{I}$ one can derive from (1.19) the proof of main conjectures of the recent paper of Givental [22]. 
As a byproduct of our computations, we obtained a nice formula for the generating function of elliptic Gromov - Witten invariants of complex projective plane. Namely, the function

$$
\psi:=\frac{\phi^{\prime \prime \prime}-27}{8\left(27+2 \phi^{\prime}-3 \phi^{\prime \prime}\right)}
$$

where

$$
\begin{aligned}
\phi(z)= & \sum_{k \geq 1} \frac{N_{k}^{(0)}}{(3 k-1) !} e^{k z}, \\
N_{k}^{(0)=} & \text { the number of rational curves of degree } k \\
& \text { on } C P^{2} \text { passing through generic } 3 k-1 \text { points }
\end{aligned}
$$

is the generating function for the numbers $N_{k}^{(1)}$ of the elliptic curves of degree $k$ on $C P^{2}$ passing through generic $3 k$ points:

$$
\psi(z)=-\frac{1}{8}+\sum_{k \geq 1} N_{k}^{(1)} \frac{k}{(3 k) !} e^{k z} .
$$

We prove also that the compatible pair of Poisson brackets describing the treelevel hierarchy admits a unique deformation to give a bihamiltonian structure, modulo $\mathcal{O}\left(\varepsilon^{4}\right)$, of the one-loop hierarchy. The deformed bihamiltonian structure turns out to be a nonlinear extension of the Virasoro algebra (i.e., a classical $W$-algebra) with the central charge

$$
c=\frac{12 \varepsilon^{2}}{(1-d)^{2}}\left[\frac{1}{2} n-2 \sum_{\alpha=1}^{n}\left(q_{\alpha}-\frac{1}{2} d\right)^{2}\right] .
$$

Here $\varepsilon$ is the string coupling constant, $d$ and $q_{\alpha}$ are the "dimension" and the "charges" of the theory. In the case of quantum cohomology of $X$ (i.e., the topological sigmamodel with the target space $X) d$ coincides with the complex dimension of the target space $X$ and $q_{\alpha}$ are the halfs of the degrees of the basic elements in $H^{*}(X)$. Remarkably, this formula works not only in quantum cohomologies. It gives the correct value for the central charge [20] of the classical $W$-algebras for the topological minimal models of A - D - E type (see below Sect.8)!

We can continue this procedure trying to construct higher genera approximation of the unknown integrable hierarchy. Of course, it would be too optimistic to expect that our procedure will go smoothly for any genus $g$ for an arbitrary solution of equations of associativity. Moreover, from [16] it follows that, constructing the integrable hierarchy, probably for a generic solution of WDVV one cannot go beyond the genus one. However, our results suggest that in an arbitrary physical 2D TFT coupling to gravity is given by an integrable bihamiltonian hierarchy of $1+1$ PDEs. Bihamiltonian structure of the hierarchy is to be described by a classical $W$-algebra with the prescribed central charge and the conformal dimensions of the primaries. So, we embed the problem of coupling to topological gravity into the problem of classification of a certain class of classical $W$-algebras. 
We briefly discuss this project in the final section, postponing the study of the higher genera corrections for a subsequent work.

The paper is organized as follows. In Section 2 we recall some important points of the theory of WDVV equations of associativity (equivalently, the theory of Frobenius manifolds) and the construction of coupling to gravity at tree-level. The main results of the paper are formulated in Section 3. In Section 4 we derive some useful identities of the theory of semisimple Frobenius manifolds used in the proof of the main results. The derivation of the bihamiltonian structure of the hierarchy in the genus one approximation is given in Section 5. In Section 6 we solve Getzler's equations for elliptic Gromov - Witten invariants for any semisimple Frobenius manifold. The examples of the deformed bihamiltonian hierarchies are given in Section 7. In the last Section 8 we discuss the programme of study of higher genera corrections in the setting of classical $W$-algebras.

Acknowledgments. The authors thank E. Getzler for fruitful discussions. We thank G.Falqui for the help with $W$-algebras. The work of one of the authors (B.D.) was done under partial support of the EC TMR Programme Integrability, non-perturbative effects and symmetry in Quantum Field Theories, grant FMRX-CT96-0012. The work of Y.Z. was supported by the Japan Society for the Promotion of Science, it was initiated in SISSA when he was a post-doc there; he thanks M. Jimbo for valuable discussions.

\section{WDVV equations of associativity and the struc- ture of a $2 \mathrm{D}$ TFT at genus zero}

WDVV equations of associativity is the problem of finding a function $F(t)=F\left(t^{1}, \ldots, t^{n}\right)$, a constant symmetric nondegenerate matrix $\left(\eta^{\alpha \beta}\right)$, numbers $q_{1}, \ldots, q_{n}, r_{1}, \ldots, r_{n}, d$ such that

$$
\partial_{\alpha} \partial_{\beta} \partial_{\lambda} F(t) \eta^{\lambda \mu} \partial_{\mu} \partial_{\gamma} \partial_{\delta} F(t)=\partial_{\delta} \partial_{\beta} \partial_{\lambda} F(t) \eta^{\lambda \mu} \partial_{\mu} \partial_{\gamma} \partial_{\alpha} F(t)
$$

for any $\alpha, \beta, \gamma, \delta=1, \ldots, n$,

$$
\begin{aligned}
& \partial_{1} \partial_{\alpha} \partial_{\beta} F(t) \equiv \eta_{\alpha \beta}, \quad \text { where }\left(\eta_{\alpha \beta}\right)=\left(\eta^{\alpha \beta}\right)^{-1}, \\
& \sum\left[\left(1-q_{\alpha}\right) t^{\alpha}+r_{\alpha}\right] \partial_{\alpha} F(t)=(3-d) F(t)+\frac{1}{2} A_{\alpha \beta} t^{\alpha} t^{\beta}+B_{\alpha} t^{\alpha}+C
\end{aligned}
$$

for some constants $A_{\alpha \beta}, B_{\alpha}, C$. The numbers $q_{\alpha}, r_{\alpha}, d$ and $A_{\alpha \beta}, B_{\alpha}, C$ must satisfy the following normalization conditions (see [12]):

$$
\begin{aligned}
& q_{1}=0, r_{\alpha} \neq 0 \quad \text { only if } q_{\alpha}=1, \\
& A_{\alpha \beta} \neq 0 \quad \text { only if } q_{\alpha}+q_{\beta}=d-1, \\
& B_{\alpha} \neq 0 \text { only if } q_{\alpha}=d-2, \\
& C \neq 0 \text { only if } d=3 \\
& A_{1 \alpha}=\sum_{\varepsilon} \eta_{\alpha \varepsilon} r_{\varepsilon}, B_{1}=0
\end{aligned}
$$


We will usually normalize the coordinates $t^{\alpha}$ reducing $\eta_{\alpha \beta}$ to the antidiagonal form

$$
\eta_{\alpha \beta}=\delta_{\alpha+\beta, n+1} .
$$

This can always be done for $d \neq 0$. Then

$$
q_{\alpha}+q_{n-\alpha+1}=d, \quad q_{n}=d .
$$

Any solution of WDVV equations provides the space of parameters $M^{n} \ni\left(t^{1}, \ldots, t^{n}\right)$ with a structure of Frobenius manifold. That means that there exists a unique structure of a Frobenius algebra $\left(A_{t},<,>\right)$ on the tangent planes $T_{t} M^{n}$ such that

$$
\left\langle\partial_{\alpha} \cdot \partial_{\beta}, \partial_{\gamma}\right\rangle=\partial_{\alpha} \partial_{\beta} \partial_{\gamma} F(t), \quad\left\langle\partial_{\alpha}, \partial_{\beta}\right\rangle=\eta_{\alpha \beta}
$$

Explicitly

$$
\partial_{\alpha} \cdot \partial_{\beta}=c_{\alpha \beta}^{\gamma}(t) \partial_{\gamma} \quad \text { where } c_{\alpha \beta}^{\gamma}(t)=\eta^{\gamma \varepsilon} \partial_{\varepsilon} \partial_{\alpha} \partial_{\beta} F(t) .
$$

The vector field

$$
e=\partial_{1}
$$

is the unity of the algebra. We introduce also the Euler vector field on $M^{n}$

$$
E(t)=E^{\varepsilon}(t) \partial_{\varepsilon}:=\sum_{\varepsilon=1}^{n}\left[\left(1-q_{\varepsilon}\right) t^{\varepsilon}+r_{\varepsilon}\right] \partial_{\varepsilon} .
$$

This is the generator of the scaling transformations (2.3). All the equations (2.1) $-(2.3)$ can be easily reformulated in a covariant way (see [11).

One of the main geometrical objects on a Frobenius manifold is a deformation of the Levi-Civita connection $\nabla$ for $<,>$ :

$$
\widetilde{\nabla}_{u} v=\nabla_{u} v+z u \cdot v .
$$

Here $u, v$ are two vector fields on $M^{n}, z$ is the parameter of the deformation. The connection (2.11) is flat for any $z$. It can be extended to a flat connection on $M^{n} \times \mathbf{C}^{*}$

$$
\widetilde{\nabla}_{\frac{d}{d z}} v=\partial_{z} v+E \cdot v-\frac{1}{z} \mu v
$$

where

$$
\begin{aligned}
& \mu:=-\nabla E+\frac{1}{2}(2-d)=\operatorname{diag}\left(\mu_{1}, \ldots, \mu_{n}\right), \quad \mu_{\alpha}=q_{\alpha}-\frac{d}{2}, \\
& \langle\mu a, b\rangle=-\langle a, \mu b\rangle .
\end{aligned}
$$

(Comparing with [1] we change the normalization of the component $\widetilde{\nabla}_{\frac{d}{d z}}$ doing an elementary gauge transform). The connection on $M^{n} \times \mathbf{C}^{*}$ is still flat.

The Frobenius manifold is said to satisfy the semisimplicity condition (or, briefly, it is semisimple) if the algebras $A_{t}$ are semisimple for generic $t$. On the open domain 
of the points of semisimplicity one can introduce canonical coordinates $u_{1}, \ldots, u_{n}$ such that

$$
\frac{\partial}{\partial u_{i}} \cdot \frac{\partial}{\partial u_{j}}=\delta_{i j} \frac{\partial}{\partial u_{i}}, i, j=1, \ldots, n
$$

(We will use all lower indices working with the canonical coordinates. No summation over the repeated indices will be assumed in this case.) In these coordinates WDVV can be reduced to a commuting family of nonstationary Hamiltonian flows on the Lie algebra $s o(n)$ with the standard Poisson bracket

$$
\frac{\partial V}{\partial u_{i}}=\left\{V, H_{i}(V ; u)\right\}, i=1, \ldots, n
$$

(the definition of the matrix $V=\left(V_{i j}\right), V^{T}=-V \in s o(n)$ see below in Sect.4), the canonical coordinates $u_{1}, \ldots, u_{n}$ play the role of the times and the quadratic Hamiltonian has the form

$$
H_{i}=\frac{1}{2} \sum_{j \neq i} \frac{V_{i j}^{2}}{u_{i}-u_{j}} .
$$

These are the equations of isomonodromy deformations of the operator

$$
\frac{d}{d z}-U-\frac{1}{z} V, \quad U=\operatorname{diag}\left(u_{1}, \ldots, u_{n}\right)
$$

with rational coefficients [9]. The tau-function $\tau_{I}$ of a solution in the theory of isomonodromy deformations is defined [24] by the quadrature

$$
d \log \tau_{I}=\sum_{i=1}^{n} H_{i} d u^{i}
$$

(We denote this function $\tau_{I}$ to avoid confusions with the tau-function (1.9) of the integrable hierarchy.)

Another geometric object is a deformation of the flat metric $<$, $>$ on $M^{n}$ [9, 11]. We introduce the intersection form

$$
\left(\omega_{1}, \omega_{2}\right)_{t}:=i_{E}\left(\omega_{1} \cdot \omega_{2}\right), \quad \omega_{1}, \omega_{2} \in T_{t}^{*} M^{n}
$$

The metric

$$
(,)_{t}-\lambda<,>_{t}
$$

on $T_{t}^{*} M^{n}$ does not degenerate for almost all $(\lambda, t)$. It is flat for these $(\lambda, t)$. In the coordinates $t^{\alpha}$

$$
g^{\alpha \beta}(t):=\left(d t^{\alpha}, d t^{\beta}\right)=E^{\varepsilon} c_{\varepsilon}^{\alpha \beta}=\left(d+1-q_{\alpha}-q_{\beta}\right) F^{\alpha \beta}(t)+A^{\alpha \beta},
$$

where

$$
F^{\alpha \beta}(t):=\eta^{\alpha \alpha^{\prime}} \eta^{\beta \beta^{\prime}} \frac{\partial^{2} F(t)}{\partial t^{\alpha^{\prime}} \partial t^{\beta^{\prime}}}, \quad A^{\alpha \beta}:=\eta^{\alpha \alpha^{\prime}} \eta^{\beta \beta^{\prime}} A_{\alpha^{\prime} \beta^{\prime}}
$$


We give also the formula for the Levi-Civita connection for the flat (but not constant in the coordinates $t^{\alpha}$ !) metric $($,

$$
\Gamma_{\gamma}^{\alpha \beta}(t):=-g^{\alpha \varepsilon}(t) \Gamma_{\varepsilon \gamma}^{\beta}(t)=\left(\frac{1+d}{2}-q_{\beta}\right) c_{\gamma}^{\alpha \beta}(t),
$$

where

$$
c_{\gamma}^{\alpha \beta}(t)=\eta^{\alpha \alpha^{\prime}} \eta^{\beta \beta^{\prime}} \partial_{\alpha^{\prime}} \partial_{\beta^{\prime}} \partial_{\gamma} F(t) .
$$

The flat metric (2.22) is responsible not only for the second Poisson bracket of the integrable hierarchy (see below), but also for the relation between Frobenius manifolds and reflection groups [11].

The genus zero approximation of the needed integrable hierarchy will be an infinite family of dynamical systems on the loop space $\mathcal{L}\left(M^{n}\right)$. We supply the loop space with a Poisson bracket

$$
\left\{v^{\alpha}(X), v^{\beta}(Y)\right\}_{1}^{(0)}=\eta^{\alpha \beta} \delta^{\prime}(X-Y),
$$

(to avoid confusions we redenote $t^{\alpha} \rightarrow v^{\alpha}$ the coordinates on $M^{n}$ when dealing with the hierarchy; comparing with the above notations of Introduction we omit the label 0 , i.e., $\left.v^{\alpha}=\eta^{\alpha \varepsilon} v_{\varepsilon}^{0}\right)$. The second Poisson bracket on the same loop space has the form

$$
\left\{v^{\alpha}(X), v^{\beta}(Y)\right\}_{2}^{(0)}=g^{\alpha \beta}(v(X)) \delta^{\prime}(X-Y)+\Gamma_{\gamma}^{\alpha \beta}(v(X)) v_{X}^{\gamma} \delta(X-Y) .
$$

Particularly, for $d \neq 1$ the Poisson bracket of

$$
T(X):=\frac{2}{1-d} t^{n}(X)
$$

has the form

$$
\{T(X), T(Y)\}_{2}^{(0)}=[T(X)+T(Y)] \delta^{\prime}(X-Y) .
$$

This coincides with the Poisson bracket on the dual space to the Lie algebra of onedimensional vector fields. Therefore the full Poisson bracket (2.27) can be considered as a nonlinear extension of this algebra (the classical W-algebra with zero central charge). Observe that

$$
\left\{t^{\alpha}(X), T(Y)\right\}_{2}^{(0)}=\left(\frac{2\left(1-q_{\alpha}\right)}{1-d} t^{\alpha}(X)+\frac{2 r_{\alpha}}{1-d}\right) \delta^{\prime}(X-Y)+t_{X}^{\alpha} \delta(X-Y)
$$

So $T(X)$ plays the role of the stress-energy tensor, and the conformal dimensions of the fields $t^{\alpha}$ having $q_{\alpha} \neq 1$ are

$$
\Delta^{\alpha}=\frac{2\left(1-q_{\alpha}\right)}{1-d}
$$

When $q_{\alpha}=1$ the variable $s^{\alpha}:=\exp t^{\alpha}$ has the Poisson bracket with the stress-energy tensor of the form

$$
\left\{s^{\alpha}(X), T(Y)\right\}_{2}^{(0)}=\frac{2 r_{\alpha}}{1-d} s^{\alpha}(X) \delta^{\prime}(X-Y)+s_{X}^{\alpha} \delta(X-Y) .
$$


So it is a primary field with the conformal dimension

$$
\Delta_{\alpha}=\frac{2 r_{\alpha}}{1-d}
$$

The two Poisson brackets are compatible, i.e., any linear combination

$$
a_{1}\{,\}_{1}^{(0)}+a_{2}\{,\}_{2}^{(0)}
$$

with arbitrary constant coefficients $a_{1}, a_{2}$ gives a Poisson bracket on $\mathcal{L}\left(M^{n}\right)$ [11]. This gives a possibility to construct a hierarchy of commuting flows on $\mathcal{L}\left(M^{n}\right)$ starting from the Casimirs of the first Poisson bracket

$$
H^{\alpha,-1}=\int v^{\alpha}(X) d X, \quad \alpha=1, \ldots, n
$$

using the standard bihamiltonian recursion procedure [29]

$$
\left\{\cdot, H^{\alpha, p}\right\}_{1}^{(0)}=k_{\alpha, p}\left\{\cdot, H^{\alpha, p-1}\right\}_{2}^{(0)}
$$

for appropriate constants $k_{\alpha, p}$. These constants are to be chosen in a clever way to make the hierarchy compatible with the genus zero recursion relations for the topological correlators. For the genus zero approximation the needed normalization of the Hamiltonians is given by an alternative procedure [9] using the flat coordinates of the deformed connection $\widetilde{\nabla}$.

The flat coordinates of $\widetilde{\nabla}$ are functions $\theta(t, z)$ such that

$$
\widetilde{\nabla} d \theta=0 .
$$

Let us forget for the moment about the last component 2.12) of the connection $\widetilde{\nabla}$. Then the flat coordinates $\theta$ are specified by the equation

$$
\partial_{\alpha} \partial_{\beta} \theta=z c_{\alpha \beta}^{\gamma} \partial_{\gamma} \theta
$$

A basis of the solutions $\theta_{1}(t, z), \ldots, \theta_{n}(t, z)$ can be obtained as power series

$$
\theta_{\gamma}(t, z)=t_{\gamma}+\sum_{p \geq 1} \theta_{\gamma, p}(t) z^{p}
$$

where the coefficients $\theta_{\gamma, p}(t)$ are determined recursively from the equations

$$
\partial_{\alpha} \partial_{\beta} \theta_{\gamma, p+1}(t)=c_{\alpha \beta}^{\rho}(t) \partial_{\rho} \theta_{\gamma, p}, \quad \theta_{\gamma, 0}(t)=t_{\gamma}=\eta_{\gamma \varepsilon} t^{\varepsilon} .
$$

One can normalize the deformed flat coordinates requiring

$$
\left\langle\nabla \theta_{\alpha}(t, z), \nabla \theta_{\beta}(t,-z)\right\rangle \equiv \eta_{\alpha \beta} .
$$

There still remains some freedom in the choice of the deformed flat coordinates

$$
\theta_{\alpha}(t, z) \mapsto \theta_{\varepsilon}(t, z) G_{\alpha}^{\varepsilon}(z)
$$


with an arbitrary matrix-valued series $G(z)=\left(G_{\alpha}^{\beta}(z)\right)$

$$
\begin{aligned}
& G(z)=1+z G_{1}+z^{2} G_{2}+\ldots \\
& G(z) \eta G(-z)^{T} \equiv \eta
\end{aligned}
$$

Later we put also the equation (2.12) into the game. This will fix the deformed flat coordinates almost uniquely.

The Hamiltonians of the genus zero hierarchy have the form

$$
H_{\beta, p}=\int \theta_{\beta, p+1}(v(X)) d X, \quad p=0,1, \ldots
$$

The hierarchy itself reads

$$
\frac{\partial v}{\partial T^{\beta, p}}=K_{\beta, p}^{(0)}\left(v, v_{X}\right)=\left\{v, H_{\beta, p}\right\}_{1}^{(0)}=\partial_{X} \nabla \theta_{\beta, p+1}(v)=\nabla \theta_{\beta, p}(v) \cdot \partial_{X} v
$$

(we treat $\partial_{X} v$ and $\partial_{T^{\beta, p}} v$ as tangent vectors to the Frobenius manifold). Observe that the coefficients in front of $\partial_{X} v$ are functions well-defined everywhere on the Frobenius manifold.

The genus zero two-point functions

$$
v_{\alpha}^{0}(T)=\left\langle\phi_{\alpha, 0} \phi_{1,0} e^{\sum T^{\beta, q} \phi_{\beta, q}}\right\rangle_{0}
$$

give a particular solution of the commutative hierarchy (2.46) specified by the following symmetry reduction

$$
\left(\partial_{T^{1,1}}-\sum_{\alpha, p} T^{\alpha, p} \partial_{T^{\alpha, p}}\right) v^{0}=0 .
$$

(I identify $T^{1,0}$ and $X$. So the variable $X$ is supressed in the formulae). The solution can be found in the form

$$
v^{0}(T)=T_{0}+\sum_{q>0} T^{\beta, q} \nabla \theta_{\beta, q}\left(T_{0}\right)+\sum_{p, q>0} T^{\beta, q} T^{\gamma, p} \nabla \theta_{\beta, q-1}\left(T_{0}\right) \cdot \nabla \theta_{\gamma, p}\left(T_{0}\right)+\ldots
$$

This is a power series in $T^{\alpha, p>0}$ with the coefficients depending on $T_{0}:=\left(T_{1,0}, \ldots, T_{n, 0}\right)$, $T_{\alpha, 0}:=\eta_{\alpha \beta} T^{\beta, 0}$. The series can be found as the fixed point $t=v^{0}$ of the gradient map $M^{n} \rightarrow M^{n}$

$$
t=\nabla \Phi_{T}(t)
$$

where

$$
\Phi_{T}(t)=\sum T^{\alpha, p} \theta_{\alpha, p}(t) .
$$

Defining the functions $\Omega_{\alpha, p ; \beta, q}(t)$ on the Frobenius manifold by the following generating function

$$
(z+w)^{-1}\left(\left\langle\nabla \theta_{\alpha}(t, z), \nabla \theta_{\beta}(t, w)\right\rangle-\eta_{\alpha \beta}\right)=\sum_{p, q=0}^{\infty} \Omega_{\alpha, p ; \beta, q}(t) z^{p} w^{q}
$$


we complete the construction of the genus zero free energy of the TFT coupled to gravity by setting

$$
\log \tau=\mathcal{F}_{0}(T)=\frac{1}{2} \sum \Omega_{\alpha, p ; \beta, q}\left(v^{0}(T)\right) \widetilde{T}^{\alpha, p} \widetilde{T}^{\beta, q},
$$

where

$$
\begin{aligned}
& \widetilde{T}^{\alpha, p}=T^{\alpha, p} \quad \text { if } \quad(\alpha, p) \neq(1,1) \\
& \widetilde{T}^{1,1}=T^{1,1}-1 .
\end{aligned}
$$

The resulting function $\mathcal{F}_{0}(T)$ satisfies the string equation

$$
\frac{\partial \mathcal{F}_{0}(T)}{\partial T^{1,0}}=\sum T^{\alpha, p} \partial_{T^{\alpha, p-1}} \mathcal{F}_{0}(T)+\frac{1}{2} \eta_{\alpha \beta} T^{\alpha, 0} T^{\beta, 0}
$$

On the small phase space $T^{\alpha, p>0}=0$ one has

$$
\begin{gathered}
\left.\mathcal{F}_{0}(T)\right|_{T^{\alpha, p>0}=0}=F(t) . \\
T^{\alpha, 0}=t^{\alpha}
\end{gathered}
$$

Also the derivatives of the function $\mathcal{F}_{0}(T)$ satisfy the genus zero recursion relations of Dijkgraaf and Witten. Observe that

$$
\frac{\partial^{2} \mathcal{F}_{0}(T)}{\partial T^{\alpha, p} \partial T^{\beta, q}}=\Omega_{\alpha, p ; \beta, q}\left(v^{0}(T)\right) .
$$

The proofs of all these results can be found in [9].

We now use the last component $\widetilde{\nabla}_{\frac{d}{d z}}$ of the deformed connection to fix the densities of the commuting Hamiltonians $H_{\alpha, p}$. Let us consider first the non-resonant case $\mu_{\alpha}-\mu_{\beta} \notin \mathbf{Z}_{\neq 0}$ for $\alpha \neq \beta$. Then the system of deformed coordinates $\tilde{t}_{\alpha}(t, z)$ of $\widetilde{\nabla}$ can be constructed in the form

$$
\begin{aligned}
& \tilde{t}_{\alpha}(t, z)=\theta_{\alpha}(t, z) z^{\mu_{\alpha}}=\sum_{p=0}^{\infty} \theta_{\alpha, p}(t) z^{p+\mu_{\alpha}}, \\
& \widetilde{\nabla}_{\frac{d}{d z}} d \tilde{t}_{\alpha}(t, z)=0 .
\end{aligned}
$$

The coefficients $\theta_{\alpha, p}(t)$ are now defined uniquely by (2.40) and by the quasihomogeneity equation following from (2.59)

$$
\mathcal{L}_{E} \theta_{\alpha, p}(t)=\left(p+\frac{2-d}{2}+\mu_{\alpha}\right) \theta_{\alpha, p}(t) .
$$

The functions $\Omega_{\alpha, p ; \beta, q}(t)$ are also quasihomogeneous of the degree $p+q+1+\mu_{\alpha}+\mu_{\beta}$. From this one easily derives the quasihomogeneity constraint for $\mathcal{F}_{0}$ (see [9]).

Let us now consider the non-generic case. We describe first the fundamental matrix solution of the linear system

$$
\widetilde{\nabla}_{\frac{d}{d z}} d \tilde{t}=0
$$


We rewrite this system for the gradient

$$
(\nabla \tilde{t})^{\alpha}=\eta^{\alpha \beta} \partial_{\beta} \tilde{t}
$$

of the deformed flat coordinates. So the columns of the fundamental matrix are the gradients of the deformed flat coordinates $\tilde{t}_{1}(t, z), \ldots, \tilde{t}_{n}(t, z)$. The fundamental matrix has the form

$$
Y(t, z)=\left(\nabla \tilde{t}_{1}(t, z), \ldots, \nabla \tilde{t}_{n}(t, z)\right)=\left(\nabla \theta_{1}(t, z), \ldots, \nabla \theta_{n}(t, z)\right) z^{\mu} z^{R},
$$

where the constant matrix $R=\left(R_{\beta}^{\alpha}\right)$ satisfies the following requirements:

1. $R_{\beta}^{\alpha} \neq 0$ only if $\mu_{\alpha}-\mu_{\beta}$ is a positive integer,

2. Let $R_{k \beta}^{\alpha}=\left\{\begin{array}{ll}R_{\beta}^{\alpha} & \text { if } \mu_{\alpha}-\mu_{\beta}=k \\ 0 & \text { otherwise }\end{array}\right.$.

We have

$$
R=R_{1}+R_{2}+\ldots
$$

(finite number of terms). Then we must have

$$
\left\langle R_{k} a, b\right\rangle+(-1)^{k}\left\langle a, R_{k} b\right\rangle=0, \quad k=1,2, \ldots
$$

for any two vectors $a, b$.

The matrix $R$ is determined uniquely up to the transformations

$$
R \mapsto G^{-1} R G,
$$

where the matrix $G=\left(G_{\beta}^{\alpha}\right)$ must satisfy the following conditions:

1. $G_{\beta}^{\alpha} \neq 0$ only if $\mu_{\alpha}-\mu_{\beta}$ is a non-negative integer.

2. Define the decomposition

$$
G=G_{0}+G_{1}+\ldots
$$

similar to (2.64). We must have

$$
G_{0}=1
$$

and the matrix $G$ must satisfy the following orthogonality condition

$$
\left\langle G^{+} a, G b\right\rangle=<a, b>
$$

for any $a, b$ where

$$
G^{+}=G_{0}-G_{1}^{T}+G_{2}^{T}-G_{3}^{T}+\ldots
$$

${ }^{1}$ Constancy of the matrix $R$ is a manifestation of the general isomonodromicity property proved in the theory of Frobenius manifolds [9, 11, 12]. 
Proof can be found in 12 . The class of equivalence of the matrix $R$ modulo the transformations (2.66) together with the matrix $\mu$ completely specifies the class of gauge equivalence of the operator $\widetilde{\nabla}_{\frac{d}{d z}}$ modulo gauge transformations of the form (2.42)-(2.44) near the singularity at $z=0$. Particularly, the coefficients $A_{\alpha \beta} B_{\alpha}, C$ in (2.3) have the form

$$
\begin{aligned}
& A_{\alpha \beta}=\eta_{\alpha \epsilon}\left(R_{1}\right)_{\beta}^{\epsilon} \\
& B_{\alpha}=\eta_{1 \varepsilon}\left(R_{2}\right)_{\alpha}^{\varepsilon}, \\
& C=-\frac{1}{2} \eta_{1 \varepsilon}\left(R_{3}\right)_{1}^{\varepsilon}
\end{aligned}
$$

Plugging the formula (2.63) into the equation $\widetilde{\nabla}_{\frac{d}{d z}}=0$ we obtain the following quasihomogeneity constraint for the function $\theta_{\alpha, p}(t)$

$$
\mathcal{L}_{E} \theta_{\alpha, p}(t)=\left(p+\frac{2-d}{2}+\mu_{\alpha}\right) \theta_{\alpha, p}(t)+\sum_{k=1}^{p} \theta_{\varepsilon, p-k}(t)\left(R_{k}\right)_{\alpha}^{\varepsilon}+\text { const } .
$$

(Observe that the functions $\theta_{\alpha, p}(t)$ are defined up to an additive constant). A more involved computation shows that

$$
\begin{aligned}
& \mathcal{L}_{E} \Omega_{\alpha, p ; \beta, q}(t)=\left(p+q+1+\mu_{\alpha}+\mu_{\beta}\right) \Omega_{\alpha, p ; \beta, q}(t)+\sum_{r=1}^{p}\left(R_{r}\right)_{\alpha}^{\varepsilon} \Omega_{\varepsilon, p-r ; \beta, q}(t) \\
& \quad+\sum_{r=1}^{q}\left(R_{r}\right)_{\beta}^{\varepsilon} \Omega_{\alpha, p ; \varepsilon, q-r}(t)+(-1)^{q}\left(R_{p+q+1}\right)_{\alpha}^{\varepsilon} \eta_{\varepsilon \beta} .
\end{aligned}
$$

Using this and the explicity formula (2.53) we arrive at

Proposition 1 The genus zero partition function $\tau$ satisfies the following constraint

$$
L_{0} \tau=0
$$

where

$$
\begin{aligned}
L_{0}= & \sum\left(\frac{1}{2}+k+\mu_{\lambda}\right) \widetilde{T}^{\lambda, k} \partial_{T^{\lambda, k}}+\sum \widetilde{T}^{\lambda, k}\left(R_{r}\right)_{\alpha}^{\varepsilon} \partial_{T^{\varepsilon, k-r}} \\
& +\frac{1}{2} \sum(-1)^{q} \widetilde{T}^{\alpha, p} \widetilde{T}^{\beta, q}\left(R_{p+q+1}\right)_{\alpha}^{\varepsilon} \eta_{\varepsilon \beta} .
\end{aligned}
$$

Here $\widetilde{T}^{\alpha, p}$ are defined by (2.54).

Example. For topological sigma-models $R$ coincides with the matrix of multiplication by the first Chern class $c_{1}(X)$ in the classical cohomologies of the target space $X$ [11]. Since $\operatorname{deg} c_{1}(X)=1$ we have

$$
R=R_{1}
$$

The recursion relation (2.74) in this case coincide with the recursion relation of Hori [23], and the particular case of (2.75) was obtained in [17]. We infer that the coefficients 
$\theta_{\alpha, p}(t)$ of the expansion of the deformed flat coordinates coincide w with the two-point functions $\left\langle\phi_{\alpha, 0} \phi_{1,0} e^{\sum_{\alpha=1}^{n} t^{\alpha} \phi_{\alpha, 0}}\right\rangle_{0}$ defined in terms of intersection theory on the moduli spaces of instantons $S^{2} \rightarrow X$. The general identity (2.76) in this particular case coincides with the $L_{0}$ Virasoro constraint derived in [23].

Remark. Applying an appropriate recursion procedure to the operator $L_{0}$ we can derive a half-infinite sequence of the Virasoro constraints

$$
L_{k} \tau=0, \quad k \geq-1
$$

generalizing the constraints of [18]. All the operators $L_{k}, k \geq-1$ are given in terms of the monodromy data $(\mu, R)$ at $z=0$. We will present these results in a separate publication.

We conclude this section with an explicit formula for the bihamiltonian structure of genus zero hierarchy (2.46).

Proposition 2 Let $(\alpha, p)$ be a pair of indices such that

$$
p+\mu_{\alpha}+\frac{1}{2} \neq 0
$$

then the equation

$$
\frac{\partial v}{\partial T^{\alpha, p}}=\left\{v, H_{\alpha, p}\right\}_{1}^{(0)}
$$

of the hierarchy (2.40) is also a Hamiltonian flow w.r.t. the second Poisson bracket (2.27)

$$
\left\{v, H_{\alpha, p}\right\}_{1}^{(0)}=\left\{v, \hat{H}_{\alpha, p}\right\}_{2}^{(0)} .
$$

The Hamiltonian $\hat{H}_{\alpha, p}$ has the form

$$
\hat{H}_{\alpha, p}=\sum_{k, l}(-1)^{k}\left(R_{p-l, k}\right)_{\alpha}^{\varepsilon} \frac{H_{\varepsilon, l-1}}{\left(p+\mu_{\alpha}+\frac{1}{2}\right)^{k+1}}
$$

where the matrices $R_{k, l}$ are defined as follows

$$
R_{0,0}=1, \quad R_{k, 0}=0 \quad \text { for } \quad k>0, R_{k, l}=\sum_{i_{1}+\ldots+i_{l}=k} R_{i_{1} \ldots R_{i_{l}}}^{\text {for }} \quad l>0 .
$$

Proof We use the identity

$$
\left\{\cdot \int \tilde{t}(v(X), z) d X\right\}_{2}^{(0)}=\left\{\cdot, \int\left(\partial_{z}-\frac{1}{2 z}\right) \tilde{t}(v(X), z) d X\right\}_{1}^{(0)}
$$

valid for an arbitrary flat coordinate $\tilde{t}$ of $\widetilde{\nabla}$ (see Lemma H.3 in [1]). Inverting, we obtain

$$
\left\{\cdot \int\left(z^{\frac{1}{2}} \int^{z} w^{-\frac{1}{2}} \tilde{t}_{\alpha}(v(X), w) d w\right) d X\right\}_{2}^{(0)}=\left\{\cdot, \int \tilde{t}_{\alpha}(v(X), z) d X\right\}_{1}^{(0)} .
$$

Integrating the expansions in both sides of the equation and using

$$
\tilde{t}_{\alpha}(t, z)=\sum \theta_{\varepsilon, p} z^{p+\mu_{\varepsilon}}\left(z^{R}\right)_{\alpha}^{\varepsilon}
$$

we obtain the formula (2.82). Proposition is proved.

\footnotetext{
${ }^{2}$ Our normalization of the correlators differs from that of Hori
} 


\section{Formulation of the main results}

We formulate now the main requirement to uniquely specify the one-loop correction to the hierarchy (2.46). We want to find a hierarchy of equations of the form

$$
\frac{\partial t}{\partial T^{\beta, p}}=K_{\beta, p}^{(0)}\left(t, t_{X}\right)+\varepsilon^{2} K_{\beta, p}^{(1)}\left(t, t_{X}, \ldots\right)
$$

such that the following property holds true (cf. [6, 16]):

Main assumption. For any solution $v=v(T)$ of the hierarchy (2.46) the function $t(T)=\left(t_{1}(T), \ldots, t_{n}(T)\right)$

$$
t(T):=v(T)+\varepsilon^{2} w(T)
$$

where

$$
w_{\alpha}(T)=\frac{\partial^{2}}{\partial T^{\alpha, 0} \partial T^{1,0}}\left\{\left[\frac{1}{24} \log \operatorname{det} M_{\alpha \beta}\left(t, t_{X}\right)+G(t)\right]_{t=v(T)}\right\}
$$

satisfies (3.1) modulo terms of the order $\varepsilon^{4}$. Here the matrix $M_{\alpha \beta}\left(t, t_{X}\right)$ is defined by (1.17), and $G(t)$ is the G-function of the Frobenius manifold (see below).

We denote $t=\left(t^{1}, \ldots, t^{n}\right)$ the dependent variables of the hierarchy to emphasize that they live on the Frobenius manifold $M^{n}$. So (3.1) is still a dynamical system on the loop space $\mathcal{L}\left(M^{n}\right)$.

It is clear that the corrections $K_{\beta, p}^{(1)}$ are determined uniquely. Indeed, the deformed hierarchy (3.1) is obtained from the tree-level hierarchy (2.46) by the infinitesimal Bäcklund transform

$$
v_{\alpha} \mapsto v_{\alpha}+\varepsilon^{2} w_{\alpha}\left(v, v_{X}, v_{X X}, v_{X X X}\right)=t_{\alpha}
$$

where the functions $w_{\alpha}$ are defined by the formula (3.3). The functions $w_{\alpha}$ are polynomials in $v_{X X}, v_{X X X}$ but they are rational functions in $v_{X}$. Remarkably, all the denominators will disappear from the deformed hierarchy.

We will prove that the corrections are polynomials in $t_{X}, t_{X X}, t_{X X X}$ for the case of semisimple Frobenius manifold (see the definition in Sect. 2 above). Observe that $M_{\alpha \beta}$ is the matrix of multiplication by the vector $\partial_{X} t$. So the determinant $\operatorname{det} M_{\alpha \beta}$ vanishes identically on the nilpotent part of the algebra $A_{t}$.

First we observe that the correction $K_{\beta, p}^{(1)}$ can be subdivided into two parts

$$
K_{\beta, p}^{(1)}=K_{\beta, p}^{\prime}+K_{\beta, p}^{\prime \prime}
$$

where $K_{\beta, p}^{\prime}$ is the contribution of the first term in the r.h.s. of (3.3), and $K_{\beta, p}^{\prime \prime}$ is the contribution of the second term respectively. The main difficulty is in the computation of $K_{\beta, p}^{\prime}$.

Theorem 1 There exists a unique hierarchy of the form

$$
\begin{aligned}
& \frac{\partial t}{\partial T^{\beta, p}}=K_{\beta, p}^{(0)}\left(t, t_{X}\right)+\varepsilon^{2}\left[K_{\beta, p ; \lambda}^{\prime}(t) t_{X X X}^{\lambda}+K_{\beta, p ; \lambda \mu}^{\prime}(t) t_{X X}^{\lambda} t_{X}^{\mu}\right. \\
& \left.\quad+K_{\beta, p ; \lambda \mu \nu}^{\prime}(t) t_{X}^{\lambda} t_{X}^{\mu} t_{X}^{\nu}\right]
\end{aligned}
$$


such that the function $t(T)=\left(t_{1}(T), \ldots, t_{n}(T)\right)$ satisfies 3.6 up to terms of order $\varepsilon^{4}$ for an arbitrary solution $v(T)$ of (2.40)

$$
t_{\alpha}(T)=v_{\alpha}(T)+\frac{\varepsilon^{2}}{24} \frac{\partial^{2}}{\partial T^{\alpha, 0} \partial T^{1,0}}\left[\log \operatorname{det} M_{\alpha \beta}\left(t, t_{X}\right)\right]_{t=v(T)} .
$$

The coefficients $K_{\beta, p ; \lambda \mu \nu}^{\prime}(t), K_{\beta, p ; \lambda \mu}^{\prime}(t), K_{\beta, p ; \lambda}^{\prime}(t)$ of the hierarchy are analytic functions on the Frobenius manifold.

The hierarchy (3.0) admits a representation

$$
\frac{\partial t}{\partial T^{\beta, p}}=\left\{t(X), H_{\beta, p}+\varepsilon^{2} \delta H_{\beta, p}^{\prime}\right\}_{1}^{\prime}+\mathcal{O}\left(\varepsilon^{4}\right)
$$

where the perturbation of the first Poisson bracket has the form

$$
\begin{aligned}
& \left\{t^{\alpha}(X), t^{\beta}(Y)\right\}_{1}^{\prime}= \\
& \quad\left\{t^{\alpha}(X), t^{\beta}(Y)\right\}_{1}^{(0)}+\frac{\varepsilon^{2}}{24}\left(\eta^{\mu \nu} c_{\mu \nu}^{\alpha \beta}(t(X))+\eta^{\mu \nu} c_{\mu \nu}^{\alpha \beta}(t(Y))\right) \delta^{\prime \prime \prime}(X-Y) \\
& \quad-\frac{\varepsilon^{2}}{24}\left[\eta^{\mu \nu} \partial_{X}^{2}\left(c_{\mu \nu}^{\alpha \beta}(t(X))\right)+\eta^{\mu \nu} \partial_{Y}^{2}\left(c_{\mu \nu}^{\alpha \beta}(t(Y))\right)\right] \delta^{\prime}(X-Y)+\mathcal{O}\left(\varepsilon^{4}\right) .
\end{aligned}
$$

The operation $\{,\}_{1}^{\prime}$ is skew-symmetric and it satisfies the Jacobi identity modulo $\mathcal{O}\left(\varepsilon^{4}\right)$. The perturbations of the Hamiltonians have the form

$$
\delta H_{\beta, p}^{\prime}=\int \chi_{\beta, p+1 ; \mu \nu}(t(X)) t_{X}^{\mu} t_{X}^{\nu} d X
$$

where $\chi_{\beta, p ; \mu \nu}=\chi_{\beta, p ; \nu \mu}$ are given by

$$
\begin{aligned}
& \chi_{\alpha, 0 ; \mu \nu}=0 \\
& \chi_{\alpha, p+1 ; \mu \nu}=\frac{1}{24} w_{\mu \nu}^{\gamma} \frac{\partial \theta_{\alpha, p}}{\partial t^{\gamma}}-\frac{1}{24} c_{\xi \zeta}^{\gamma} c_{\nu}^{\xi \sigma} c_{\sigma \mu}^{\zeta} \frac{\partial \theta_{\alpha, p-1}}{\partial t^{\gamma}}, \quad p \geq 0 .
\end{aligned}
$$

Here $\theta_{\alpha,-1}=0$ and $w_{\mu \nu}^{\alpha}$ are defined by

$$
w_{\beta \gamma}^{\alpha}=c_{\mu \beta}^{\mu \nu} c_{\nu \gamma}^{\alpha}-c_{\gamma}^{\mu \nu} c_{\mu \nu \beta}^{\alpha}=c_{\mu \beta}^{\mu \nu} c_{\nu \gamma}^{\alpha}+c_{\mu \gamma}^{\mu \nu} c_{\nu \beta}^{\alpha}-\partial_{\mu}\left(c_{\beta \gamma}^{\nu} c_{\nu}^{\alpha \mu}\right)
$$

The Hamiltonians $H_{\beta, p}+\varepsilon^{2} \delta H_{\beta, p}^{\prime}$ commute pairwise modulo $\mathcal{O}\left(\varepsilon^{4}\right)$ w.r.t. the bracket (3.9).

Here and below $c_{\gamma \mu}^{\alpha}, c_{\gamma \mu \nu}^{\alpha}, c_{\gamma \mu \nu \sigma}^{\alpha}, c_{\gamma \mu}^{\alpha \beta}, c_{\gamma \mu \nu}^{\alpha \beta}, c_{\gamma \mu \nu \sigma}^{\alpha \beta}$ are obtained by taking derivatives of the function $F\left(t^{1}, \ldots, t^{N}\right)$ with respect to the coordinates $t^{1}, \ldots, t^{N}$ and by using $\eta^{\alpha \beta}$ to raise the indices, for example,

$$
c_{\gamma \mu}^{\alpha \beta}=\eta^{\alpha \alpha^{\prime}} \eta^{\beta \beta^{\prime}} \frac{\partial^{4} F(t)}{\partial t^{\alpha^{\prime}} \partial t^{\beta^{\prime}} \partial t^{\gamma} \partial t^{\mu}} .
$$

Remark. The first theorem does not use the quasihomogeneity condition (2.3). The next theorem does use it. 
Theorem 2 The following formulae give the perturbation of the second Poisson bracket:

$$
\begin{aligned}
& \left\{t^{\alpha}(X), t^{\beta}(Y)\right\}_{2}^{\prime}=\left\{t^{\alpha}(X), t^{\beta}(Y)\right\}_{2}^{(0)} \\
& \quad+\varepsilon^{2}\left[h^{\alpha \beta}(t(X)) \delta^{\prime \prime \prime}(X-Y)+r_{\gamma}^{\alpha \beta}(t(X)) t_{X}^{\gamma} \delta^{\prime \prime}(X-Y)\right. \\
& \quad+\left(f_{\gamma}^{\alpha \beta}(t(X)) t_{X X}^{\gamma}+q_{\gamma \mu}^{\alpha \beta}(t(X)) t_{X}^{\gamma} t_{X}^{\mu}\right) \delta^{\prime}(X-Y) \\
& \left.\quad+\left(b_{\gamma \mu}^{\alpha \beta}(t(X)) t_{X}^{\gamma} t_{X X}^{\mu}+a_{\gamma \mu \nu}^{\alpha \beta}(t(X)) t_{X}^{\gamma} t_{X}^{\mu} t_{X}^{\nu}+p_{\gamma}^{\alpha \beta}(t(X)) t_{X X X}^{\gamma}\right) \delta(X-Y)\right] \\
& \quad+\mathcal{O}\left(\varepsilon^{4}\right) .
\end{aligned}
$$

where

$$
\begin{aligned}
h^{\alpha \beta}= & \frac{1}{12}\left(\partial_{\nu}\left(g^{\mu \nu} c_{\mu}^{\alpha \beta}\right)+\frac{1}{2} c_{\nu}^{\mu \nu} c_{\mu}^{\alpha \beta}\right), \\
p_{\gamma}^{\alpha \beta}= & \frac{1}{12}\left(\frac{1}{2}-\mu_{\beta}\right) c_{\mu \nu}^{\alpha \beta} c_{\gamma}^{\mu \nu}, \\
a_{\gamma \mu \nu}^{\alpha \beta}= & \frac{1}{72}\left(\frac{1}{2}-\mu_{\beta}\right)\left(\eta ^ { \alpha \sigma } \left(\partial_{\sigma} \partial_{\nu} w_{\gamma \mu}^{\beta}+\partial_{\sigma} \partial_{\gamma} w_{\mu \nu}^{\beta}\right.\right. \\
& \left.+\partial_{\sigma} \partial_{\mu} w_{\gamma \nu}^{\beta}-2 \partial_{\mu} \partial_{\nu} w_{\gamma \sigma}^{\beta}-2 \partial_{\mu} \partial_{\gamma} w_{\nu \sigma}^{\beta}-2 \partial_{\nu} \partial_{\gamma} w_{\mu \sigma}^{\beta}\right) \\
& +\eta^{\xi \zeta}\left(6 c_{\xi \zeta}^{\alpha \sigma} c_{\sigma \gamma \mu \nu}^{\beta}+3 c_{\xi \zeta \gamma}^{\alpha \sigma} c_{\sigma \mu \nu}^{\beta}+3 c_{\xi \zeta \mu}^{\alpha \sigma} c_{\sigma \gamma \nu}^{\beta}\right. \\
& \left.\left.+3 c_{\xi \zeta \nu}^{\alpha \sigma} c_{\sigma \gamma \mu}^{\beta}+c_{\xi \zeta \gamma \mu}^{\alpha \sigma} c_{\sigma \nu}^{\beta}+c_{\xi \zeta \gamma \nu}^{\alpha \sigma} c_{\sigma \mu}^{\beta}+c_{\xi \zeta \mu \nu}^{\alpha \sigma} c_{\sigma \gamma}^{\beta}\right)\right), \\
b_{\gamma \mu}^{\alpha \beta}= & \frac{1}{12}\left(\frac{1}{2}-\mu_{\beta}\right)\left(\eta^{\alpha \sigma}\left(-2 \partial_{\gamma} w_{\mu \sigma}^{\beta}+\partial_{\sigma} w_{\gamma \mu}^{\beta}-\partial_{\mu} w_{\gamma \sigma}^{\beta}\right)\right. \\
& \left.+\eta^{\xi \zeta}\left(3 c_{\xi \zeta}^{\alpha \sigma} c_{\sigma \gamma \mu}^{\beta}+\frac{3}{2} c_{\xi \zeta \gamma}^{\alpha \sigma} c_{\sigma \mu}^{\beta}+\frac{1}{2} c_{\xi \zeta \mu}^{\alpha \sigma} c_{\sigma \gamma}^{\beta}\right)\right) \\
& \frac{3}{2} \partial_{\gamma} h^{\alpha \beta}+\frac{1}{24}\left(\frac{3}{2}-\mu_{\beta}\right) c_{\gamma}^{\alpha \nu} c_{\nu \mu}^{\beta \mu}-\frac{1}{24}\left(\frac{3}{2}-\mu_{\alpha}\right) c_{\gamma}^{\beta \nu} c_{\nu \mu}^{\alpha \mu}, \\
r_{\gamma}^{\alpha \beta}, & r_{\gamma}^{\alpha \beta}+p_{\gamma}^{\alpha \beta}+p_{\gamma}^{\beta \alpha}-\partial_{\gamma} h^{\alpha \beta}, \\
f_{\gamma}^{\alpha \beta}= & \frac{1}{2}\left(b_{\mu \gamma}^{\alpha \beta}+b_{\mu \gamma}^{\beta \alpha}\right)+\frac{1}{2} \partial_{\gamma} r_{\mu}^{\alpha \beta}+\frac{1}{2} \partial_{\mu} r_{\gamma}^{\alpha \beta}-\partial_{\mu} \partial_{\gamma} h^{\alpha \beta}- \\
q_{\gamma \mu}^{\alpha \beta}= & \frac{1}{2} \partial_{\mu}\left(p_{\gamma}^{\alpha \beta}+p_{\gamma}^{\beta \alpha}\right) .
\end{aligned}
$$

The Jacobi identity for a linear combination

$$
a_{1}\{,\}_{1}^{\prime}+a_{2}\{,\}_{2}^{\prime}
$$

with arbitrary constant coefficients $a_{1}, a_{2}$ holds true modulo $\mathcal{O}\left(\varepsilon^{4}\right)$. The equations of the perturbed hierarchy for those $(\beta, p)$ for which

$$
p+\mu_{\beta}+\frac{1}{2} \neq 0
$$

are Hamiltonian flows also w.r.t. the second Poisson bracket (3.14) with the Hamiltonian

$$
\hat{H}_{\beta, p}^{\prime}=\sum_{k, l}(-1)^{k}\left(R_{p-l, k}\right)_{\beta}^{\epsilon} \frac{H_{\epsilon, l-1}+\varepsilon^{2} \delta H_{\epsilon, l-1}^{\prime}}{\left(p+\mu_{\alpha}+\frac{1}{2}\right)^{k+1}} .
$$

Here $R_{l, k}$ are defined in (2.84). 
The proofs will be given in Sect. 5. The deformations (3.9) and (3.14) of the Poisson brackets are obtained by applying the same infinitesimal Bäcklund transform (3.7) to the Poisson brackets (2.26) and (2.27) resp. We prove that after this transform each of the deformed Poisson bracket is a combination of $\delta(X-Y), \ldots, \delta^{\prime \prime \prime}(X-Y)$ with the coefficients being polynomial in $t_{X}, t_{X X}, t_{X X X}$. Coefficients of these polynomials are functions analytic on the Frobenius manifold. (Assuming the Frobenius manifold to be analytic itself). Applying similar procedure to the Hamiltonians (2.45) we obtained the deformed Hamiltonians (3.10). The structure (3.6) of the deformed hierarchy follows from the fromulae (3.8)-(3.11). Finally, the same infinitesimal Bäcklund transform gives the deformation of the linear pencil (2.34) of the Poisson brackets.

We describe now the effect of adding of the second term in the formula (3.3). At the moment we consider $G(t)$ as an arbitrary function on some domain in the Frobenius manifold. We will compute this function in Theorem 3 below.

Proposition 3 Inserting an arbitrary function $G(t)$ in (3.3) we preserve the structure of the hierarchy, of the Hamiltonian, and of the Poisson brackets. The Hamiltonians get a correction $\varepsilon^{2} \delta H_{\alpha, p}^{\prime \prime}$ with

$$
\delta H_{\alpha, p}^{\prime \prime}=c_{\xi \nu}^{\gamma} c_{\mu}^{\sigma \xi} \frac{\partial \theta_{\alpha, p}}{\partial t^{\gamma}} \frac{\partial G}{\partial t^{\sigma}}
$$

The deformations of the first and of the second Poisson brackets get the correction $\varepsilon^{2}\{,\}_{1}^{\prime \prime}$ and $\varepsilon^{2}\{,\}_{1}^{\prime \prime}$ with

$$
\begin{aligned}
& \left\{t^{\alpha}(X), t^{\beta}(Y)\right\}_{1}^{\prime \prime}= \\
& \quad \tilde{a}^{\alpha \beta}(t(X)) \delta^{\prime \prime \prime}(X-Y)+\tilde{b}^{\alpha \beta}(t(X)) \delta^{\prime \prime}(X-Y) \\
& \quad+\tilde{e}^{\alpha \beta}(t(X)) \delta^{\prime}(X-Y), \\
& \left\{t^{\alpha}(X), t^{\beta}(Y)\right\}_{2}^{\prime \prime}= \\
& \quad a^{\alpha \beta}(t(X)) \delta^{\prime \prime \prime}(X-Y)+b^{\alpha \beta}(t(X)) \delta^{\prime \prime}(X-Y) \\
& +e^{\alpha \beta}(t(X)) \delta^{\prime}(X-Y)+\partial_{X}\left(q^{\alpha \beta}(t(X))\right) \delta(X-Y),
\end{aligned}
$$

where

$$
\begin{aligned}
\tilde{a}^{\alpha \beta} & =2 c^{\alpha \beta \mu} \frac{\partial G(t)}{\partial t^{\mu}} \\
\tilde{b}^{\alpha \beta} & =\frac{3}{2} \partial_{X} \tilde{a}^{\alpha \beta}+\frac{\partial^{2} G}{\partial t^{\sigma} \partial t^{\rho}}\left(c_{\mu}^{\alpha \sigma} \eta^{\beta \rho}-c_{\mu}^{\beta \sigma} \eta^{\alpha \rho}\right) t_{X}^{\mu} \\
\tilde{e}^{\alpha \beta} & =\partial_{X} \tilde{b}^{\alpha \beta}-\partial_{X}^{2} \tilde{a}^{\alpha \beta} \\
a^{\alpha \beta} & =2 c_{\gamma}^{\alpha \mu} g^{\gamma \beta} \frac{\partial G(t)}{\partial t^{\mu}} \\
b^{\alpha \beta} & =\frac{3}{2} \partial_{X} a^{\alpha \beta}+\left(c_{\mu}^{\alpha \rho} g^{\gamma \beta}-c_{\mu}^{\beta \rho} g^{\gamma \alpha}\right) \frac{\partial^{2} G(t)}{\partial t^{\gamma} \partial t^{\rho}} t_{X}^{\mu}+\left(\mu_{\alpha}-\mu_{\beta}\right) c_{\gamma}^{\alpha \rho} c_{\mu}^{\gamma \beta} \frac{\partial G(t)}{\partial t^{\rho}} t_{X}^{\mu}, \\
q^{\alpha \beta} & =\left(\frac{1}{2}-\mu_{\beta}\right)\left(\frac{\partial^{2} G}{\partial t^{\sigma} \partial t^{\rho}} c_{\mu}^{\alpha \sigma} c_{\nu}^{\beta \rho}-\frac{\partial^{2} G}{\partial t^{\sigma} \partial t^{\nu}} c_{\rho}^{\beta \sigma} c_{\mu}^{\rho \alpha}+\frac{\partial G}{\partial t^{\sigma}} c_{\rho \mu}^{\alpha \beta} c_{\nu}^{\rho \sigma}\right) t_{X}^{\mu} t_{X}^{\nu}, \\
e^{\alpha \beta} & =q^{\alpha \beta}+q^{\beta \alpha}+\partial_{X} b^{\alpha \beta}-\partial_{X}^{2} a^{\alpha \beta} .
\end{aligned}
$$


The full Poisson brackets of the one-loop deformed hierarchy are $\{,\}_{1}=\{,\}_{1}^{\prime}+$ $\{,\}_{1}^{\prime \prime}$ and $\{,\}_{2}=\{,\}_{2}^{\prime}+\{,\}_{2}^{\prime \prime}$.

For the case of quantum cohomology the function $G(t)$ must be the generating function of the elliptic Gromov - Witten invariants of the target space. The recursion relations for the elliptic Gromov - Witten invariants were found by Getzler [21]. He proved that the generating function $G(t)$ must satisfy a complicated system of differential equations (see (6.1) below). This system makes sense on an arbitrary Frobenius manifold. Our next result is the solution of this system on an arbitrary semisimple Frobenius manifolds.

Theorem 3 For an arbitrary semisimple Frobenius manifold the system (6.1) has a unique, up to an additive constant, solution $G=G\left(t^{2}, \ldots, t^{n}\right)$ satisfying the quasihomogeneity condition

$$
\mathcal{L}_{E} G=\gamma
$$

with a constant $\gamma$. This solution is given by the formula

$$
G=\log \frac{\tau_{I}}{J^{1 / 24}}
$$

where $\tau_{I}$ is the isomonodromic tau-function (2.19) and

$$
J=\operatorname{det}\left(\frac{\partial t^{\alpha}}{\partial u^{i}}\right)
$$

is the Jacobian of the transform from the canonical coordinates to the flat ones. The scaling anomaly $\gamma$ in (3.29) is given by the formula

$$
\gamma=-\frac{1}{4} \sum_{\alpha=1}^{n} \mu_{\alpha}^{2}+\frac{n d}{48}
$$

where

$$
\mu_{\alpha}=q_{\alpha}-\frac{d}{2}, \alpha=1, \ldots, n .
$$

Corollary 1 For $d \neq 1$ the variable $T(X)=\frac{2}{1-d} t^{n}(X)$ has the Virasoro Poisson bracket

$$
\{T(X), T(Y)\}_{2}=[T(X)+T(Y)] \delta^{\prime}(X-Y)+\varepsilon^{2} \frac{c}{12} \delta^{\prime \prime \prime}(X-Y)
$$

with the central charge

$$
c \varepsilon^{2}=\frac{12 \varepsilon^{2}}{(1-d)^{2}}\left[\frac{1}{2} n-2 \sum_{\alpha=1}^{n} \mu_{\alpha}^{2}\right] .
$$

So, the bihamiltonian structure of the conjectured integrable hierarchy at the oneloop approximation looks like a classical $W$-algebra with the central charge (3.35) and the conformal weights (2.31). 


\section{Some formulas related to the canonical coordi- nates of Frobenius manifold}

The canonical coordinates on a semisimple Frobenius manifold $M^{n}$ are denoted by $\left(u_{1}, \ldots, u_{n}\right)$. They satisfy the multiplication table

$$
\frac{\partial}{\partial u_{i}} \cdot \frac{\partial}{\partial u_{j}}=\delta_{i j} \frac{\partial}{\partial u_{i}}
$$

The invariant metric becomes diagonal in the canonical coordinates, i.e. $<,>=$ $\sum_{i=1}^{n} \eta_{i i}(u) d u_{i}^{2}$. We assume that the unit vector field of the Frobenius manifold is $e=\frac{\partial}{\partial t^{1}}$, then the rotation coefficients $\gamma_{i j}(u)$ are defined by

$$
\gamma_{i j}(u)=\frac{\partial_{j} \sqrt{\eta_{i i}(u)}}{\sqrt{\eta_{j j}(u)}}=\frac{1}{2} \frac{\partial_{i} \partial_{j} t_{1}(u)}{\sqrt{\partial_{i} t_{1}(u) \partial_{j} t_{1}(u)}}, \quad \text { for } i \neq j
$$

where $\partial_{i}=\frac{\partial}{\partial u_{i}}$. They are symmetric with respect to their indices and satisfy the following equations:

$$
\begin{aligned}
& \frac{\partial \gamma_{i j}}{\partial u_{k}}=\gamma_{i k} \gamma_{k j}, \quad i, j, k \text { distinct, } \\
& \sum_{k=1}^{N} \frac{\partial \gamma_{i j}}{\partial u_{k}}=0
\end{aligned}
$$

Define

$$
\psi_{i \alpha}(u)=\frac{\partial_{i} t_{\alpha}(u)}{\sqrt{\eta_{i i}(u)}} .
$$

The matrix $\left(\psi_{i \alpha}\right)$ satisfies the following identities:

$$
\sum_{l=1}^{n} \psi_{l \alpha} \psi_{l \beta}=\eta_{\alpha \beta}, \quad \sum_{l=1}^{n} \psi_{l}^{\alpha} \psi_{l}^{\beta}=\eta^{\alpha \beta}
$$

where $\psi_{j}^{\alpha}=\psi_{j \gamma} \eta^{\gamma \beta}$. We list here the following useful identities: (see [1])

$$
\begin{aligned}
c_{\alpha \beta \gamma} & =\sum_{i=1}^{n} \frac{\psi_{i \alpha} \psi_{i \beta} \psi_{i \gamma}}{\psi_{i 1}} \\
\frac{\partial t^{\alpha}}{\partial u_{i}} & =\psi_{i 1} \psi_{i}^{\alpha}, \quad \frac{\partial u_{i}}{\partial t^{\alpha}}=\frac{\psi_{i \alpha}}{\psi_{i 1}} \\
\frac{\partial \psi_{i \alpha}}{\partial u_{k}} & =\gamma_{i k} \psi_{k \alpha}, \quad i \neq k, \quad \frac{\partial \psi_{i \alpha}}{\partial u_{i}}=-\sum_{k} \gamma_{i k} \psi_{k \alpha} \\
\frac{\partial \psi_{i \alpha}}{\partial t^{\beta}} & =\sum_{l=1}^{N} \gamma_{i l} \psi_{l \alpha}\left(\frac{\psi_{l \beta}}{\psi_{l 1}}-\frac{\psi_{i \beta}}{\psi_{i 1}}\right)
\end{aligned}
$$


Denote

$$
\sigma_{i}=\psi_{i \alpha} t_{X}^{\alpha}
$$

Then we have

$$
\frac{\partial \sigma_{i}}{\partial t^{\alpha}}=\sum_{l=1}^{n} \gamma_{i l} \sigma_{l}\left(\frac{\psi_{l \alpha}}{\psi_{l 1}}-\frac{\psi_{i \alpha}}{\psi_{i 1}}\right)
$$

Let's consider the matrix

$$
A=\left(\psi_{i}^{\alpha} \psi_{j}^{\beta} c_{\alpha \beta \gamma} t_{X}^{\gamma}\right)
$$

by using the identity (4.6) we have

$$
\operatorname{det} A=\operatorname{det}\left(\eta^{\alpha \beta}\right) \operatorname{det}\left(c_{\alpha \beta \gamma} t_{X}^{\gamma}\right)
$$

We see that the matrix $c_{\alpha \beta \gamma} t_{X}^{\gamma}$ diagonalizes in the canonical coordinates. From (4.7) we see that the following expression for $\mathcal{F}^{(1)}\left(t, t_{X}\right)$ holds true:

$$
\begin{aligned}
& \mathcal{F}^{(1)}\left(t, t_{X}\right)=\frac{1}{24} \log \operatorname{det}\left(c_{\alpha \beta \gamma} t_{X}^{\gamma}\right)+G(t) \\
= & -\frac{1}{24} \log \operatorname{det}\left(\eta^{\alpha \beta}\right)+\frac{1}{24} \log \operatorname{det}(A)+G(t) \\
= & -\frac{1}{24} \log \operatorname{det}\left(\eta^{\alpha \beta}\right)+\frac{1}{24} \log \operatorname{det}\left(\psi_{i}^{\alpha} \psi_{j}^{\beta} \sum_{k} \frac{\psi_{k \alpha} \psi_{k \beta} \psi_{k \gamma}}{\psi_{k 1}} t_{X}^{\gamma}\right)+G(t) \\
= & \frac{1}{24} \log \left(\prod_{l=1}^{n} \psi_{l \gamma} t_{X}^{\gamma}\right)-\frac{1}{24} \log \left(\prod_{l=1}^{n} \psi_{l 1}\right)+G(t)-\frac{1}{24} \log \operatorname{det}\left(\eta^{\alpha \beta}\right), \\
= & \frac{1}{24} \log \prod_{l=1}^{n} \sigma_{l}-\frac{1}{24} \log \left(\prod_{l=1}^{n} \psi_{l 1}\right)+G(t)-\frac{1}{24} \log \operatorname{det}\left(\eta^{\alpha \beta}\right)
\end{aligned}
$$

This expression of the function $\mathcal{F}^{(1)}$ is crucial in the proof of Theorems 1,2 .

Let's denote

$$
\begin{aligned}
& F^{(1)}:=\frac{1}{24} \log \operatorname{det}\left(c_{\alpha \beta \gamma} t_{X}^{\gamma}\right) \\
& =\frac{1}{24} \log \prod_{l=1}^{n} \sigma_{l}-\frac{1}{24} \log \left(\prod_{l=1}^{n} \psi_{l 1}\right)-\frac{1}{24} \log \operatorname{det}\left(\eta^{\alpha \beta}\right) .
\end{aligned}
$$

By a direct calculation we get also the following formulas:

$$
\begin{aligned}
F_{t_{X}^{\alpha}}^{(1)}= & \frac{1}{24} \sum_{i=1}^{n} \frac{\psi_{i \alpha}}{\sigma_{i}} \\
F_{t_{X}^{\alpha} t_{X}^{\beta}}^{(1)}= & -\frac{1}{24} \sum_{i=1}^{n} \frac{\psi_{i \alpha} \psi_{i \beta}}{\sigma_{i}^{2}} \\
F_{t_{X}^{\alpha} t^{\beta}}^{(1)}= & \frac{1}{24} \sum_{j, k=1}^{n} \frac{\gamma_{j k} \psi_{k \alpha}}{\sigma_{j}}\left(\frac{\psi_{k \beta}}{\psi_{k 1}}-\frac{\psi_{j \beta}}{\psi_{j 1}}\right) \\
& -\frac{1}{24} \sum_{j, k, \gamma=1}^{N} \frac{\gamma_{j k} \sigma_{k} \psi_{j \alpha}}{\sigma_{j}^{2}}\left(\frac{\psi_{k \beta}}{\psi_{k 1}}-\frac{\psi_{j \beta}}{\psi_{j 1}}\right),
\end{aligned}
$$




$$
\begin{aligned}
F_{t^{\alpha}}^{(1)}= & \frac{1}{24} \sum_{i, j=1}^{N} \gamma_{i j}\left(\frac{\sigma_{j}}{\sigma_{i}}\left(\frac{\psi_{j \alpha}}{\psi_{j 1}}-\frac{\psi_{i \alpha}}{\psi_{i 1}}\right)-\frac{\psi_{j \alpha}}{\psi_{i 1}}+\frac{\psi_{i \alpha} \psi_{j 1}}{\psi_{i 1}^{2}}\right) \\
c_{\beta \mu}^{\alpha \gamma}= & \sum_{i, j=1}^{N} \gamma_{i j}\left(\frac{\psi_{i}^{\alpha} \psi_{i \beta} \psi_{j}^{\gamma}}{\psi_{i 1}}+\frac{\psi_{i}^{\alpha} \psi_{i}^{\gamma} \psi_{j \beta}}{\psi_{i 1}}+\frac{\psi_{i}^{\gamma} \psi_{i \beta} \psi_{j}^{\alpha}}{\psi_{i 1}}-\frac{\psi_{i}^{\gamma} \psi_{i}^{\alpha} \psi_{i \beta} \psi_{j 1}}{\psi_{i 1}^{2}}\right) \\
& \times\left(\frac{\psi_{j \mu}}{\psi_{j 1}}-\frac{\psi_{i \mu}}{\psi_{i 1}}\right) .
\end{aligned}
$$

All these formulae do not use the quasihomogeneity (2.3). In the quasihomogeneous case the canonical coordinates $u_{1}(t), \ldots, u_{n}(t)$ are the roots of the characteristic equation

$$
\operatorname{det}\left(g^{\alpha \beta}(t)-u \eta^{\alpha \beta}\right)=0 .
$$

Here $g^{\alpha \beta}$ is the intersection form. The matrix $\gamma_{i j}$ in this case has the form

$$
\gamma_{i j}=-\left(u_{i}-u_{j}\right)^{-1} V_{i j}
$$

where

$$
V_{i j}=\sum_{\alpha=1}^{n} \mu_{\alpha} \psi_{i \alpha} \psi_{j}^{\alpha}
$$

The columns of the matrix $\Psi=\left(\psi_{i \alpha}\right)$ are the eigenvectors of the matrix $V$ with the eigenvalues $\mu_{\alpha}$. Particularly, $\psi_{i 1}$ is the eigenvector of $V$ with the eigenvalue $\mu_{1}=-d / 2$. It follows that

$$
\begin{gathered}
\partial_{k} \gamma_{i j}=\gamma_{i k} \gamma_{k j}, k \neq i, j, \quad \partial_{i} \gamma_{i j}=\frac{\sum_{k}\left(u_{j}-u_{k}\right) \gamma_{i k} \gamma_{k j}-\gamma_{i j}}{u_{i}-u_{j}} \\
\frac{\partial \gamma_{i j}}{\partial t^{\alpha}}=\sum_{k=1}^{N}\left(\gamma_{i k} \gamma_{k j} \frac{\psi_{k \alpha}}{\psi_{k 1}}+\frac{u_{j}-u_{k}}{u_{i}-u_{j}} \gamma_{i k} \gamma_{k j} \frac{\psi_{i \alpha}}{\psi_{i 1}}+\frac{u_{i}-u_{k}}{u_{j}-u_{i}} \gamma_{i k} \gamma_{k j} \frac{\psi_{j \alpha}}{\psi_{j 1}}\right. \\
\left.-\frac{\gamma_{i j}}{u_{i}-u_{j}} \frac{\psi_{i \alpha}}{\psi_{i 1}}-\frac{\gamma_{i j}}{u_{j}-u_{i}} \frac{\psi_{j \alpha}}{\psi_{j 1}}\right) .
\end{gathered}
$$

We also write down the following useful formulae:

$$
\begin{aligned}
& \psi_{i \gamma} g^{\gamma \beta}=u_{i} \psi_{i}^{\beta}, \\
& \left(u^{j}-u^{i}\right) \gamma_{i j}=\sum_{\alpha}\left(q_{\alpha}-\frac{d}{2}\right) \psi_{i \alpha} \psi_{j}^{\alpha} .
\end{aligned}
$$

\section{Proofs of theorem 1 and 2}

We begin with the proof of Theorem 1. So we assume here that $G=0$ in the formulae (3.2), (3.3). Doing the infinitesimal Bäcklund tranform (3.2) (with $G(t)=0$ ) we obtain

$$
\left\{t^{\alpha}(X), t^{\beta}(Y)\right\}_{1}^{\prime}=\eta^{\alpha \beta} \delta^{\prime}(X-Y)
$$




$$
\begin{aligned}
& +\varepsilon^{2}\left[\left(\frac{\partial w^{\beta}(t(Y))}{\partial t^{\gamma}}+\frac{\partial w^{\beta}(t(Y))}{\partial t_{Y}^{\gamma}} \partial_{Y}+\frac{\partial w^{\beta}(t(Y))}{\partial t_{X X}^{\gamma}} \partial_{X}^{2}\right.\right. \\
& \left.+\frac{\partial w^{\beta}(t(Y))}{\partial t_{Y Y Y}^{\gamma}} \partial_{Y}^{3}\right) \eta^{\alpha \gamma} \delta^{\prime}(X-Y)+\left(\frac{\partial w^{\alpha}(t(X))}{\partial t^{\gamma}}+\frac{\partial w^{\alpha}(t(X))}{\partial t_{X}^{\gamma}} \partial_{X}\right. \\
& \left.\left.+\frac{\partial w^{\alpha}(t(X))}{\partial t_{X X}^{\gamma}} \partial_{X}^{2}+\frac{\partial w^{\alpha}(t(X))}{\partial t_{X X X}^{\gamma}} \partial_{X}^{3}\right) \eta^{\gamma \beta} \delta^{\prime}(X-Y)\right]+\mathcal{O}\left(\varepsilon^{4}\right)
\end{aligned}
$$

where $w^{\alpha}(t)=w^{\alpha}\left(t, t_{X}\right)$ is the function obtained from $w^{\alpha}\left(v, v_{X}\right)$ by replacing $v^{\mu}$ and their $X$-derivatives by $t^{\mu}$ and by the correspondent $X$-derivatives of $t^{\mu}$. Recall that $w^{\alpha}(t)$ depends not only on $t^{\alpha}$, but also on $t_{X}^{\alpha}, t_{X X}^{\alpha}, t_{X X X}^{\alpha}$. More explicitly, we have

$$
\begin{aligned}
w^{\alpha}(t)= & F_{t_{X}^{\beta}}^{(1)} c_{\gamma}^{\alpha \beta} t_{X X X}^{\gamma}+\left(F_{t_{X}^{\gamma} t^{\beta}}^{(1)} c_{\mu}^{\alpha \beta}+F_{t_{X}^{\beta} t^{\mu}}^{(1)} c_{\gamma}^{\alpha \beta}+3 F_{t_{X}^{\beta}}^{(1)} c_{\gamma \mu}^{\alpha \beta}\right) t_{X X}^{\gamma} t_{X}^{\mu} \\
& +F_{t_{X}^{\beta} t_{X}^{\mu}}^{(1)} c_{\gamma}^{\alpha \beta} t_{X X}^{\gamma} t_{X X}^{\mu}+F_{t_{X}^{\beta} t_{X}^{\nu}}^{(1)} c_{\gamma \mu}^{\alpha \beta} t_{X}^{\gamma} t_{X}^{\mu} t_{X X}^{\nu}+F_{t^{\beta}}^{(1)} c_{\gamma}^{\alpha \beta} t_{X X}^{\gamma} \\
& +\left(F_{t_{X}^{\beta} t^{\nu}}^{(1)} c_{\gamma \mu}^{\alpha \beta}+F_{t_{X}^{\beta}}^{(1)} c_{\gamma \mu \nu}^{\alpha \beta}\right) t_{X}^{\gamma} t_{X}^{\mu} t_{X}^{\nu}+\left(F_{t^{\beta} t^{\mu}}^{(1)} c_{\gamma}^{\alpha \beta}+F_{t^{\beta}}^{(1)} c_{\gamma \mu}^{\alpha \beta}\right) t_{X}^{\gamma} t_{X}^{\mu},
\end{aligned}
$$

where $F^{(1)}=F^{(1)}\left(t, t_{X}\right)$ is defined in (4.16). Whenever there is no risk of confusion we will omit the arguments of a function henceforth.

In the Poisson bracket $\left\{t^{\alpha}(X), t^{\beta}(Y)\right\}_{1}^{\prime}$, the coefficient of $\varepsilon^{2} \delta^{(4)}(X-Y)$ is equal to zero, so it can be written as

$$
\begin{aligned}
& \left\{t^{\alpha}(X), t^{\beta}(Y)\right\}_{1}^{\prime}=\eta^{\alpha \beta} \delta^{\prime}(X-Y)+\varepsilon^{2}\left(\hat{h}^{\alpha \beta} \delta^{(3)}(X-Y)\right. \\
& \left.+\hat{r}^{\alpha \beta} \delta^{\prime \prime}(X-Y)+\hat{f}^{\alpha \beta} \delta^{\prime}(X-Y)+\hat{p}^{\alpha \beta} \delta(X-Y)\right)
\end{aligned}
$$

where $\hat{h}^{\alpha \beta}, \hat{r}^{\alpha \beta}, \hat{f}^{\alpha \beta}, \hat{p}^{\alpha \beta}$ are functions of $t^{\mu}(X)$ and their $X$-derivatives.

We have the following two lemmas on the coefficients $\hat{h}^{\alpha \beta}$ and $\hat{r}^{\alpha \beta}$ :

Lemma 1 The coefficients $\hat{h}^{\alpha \beta}$ have the expression

$$
\hat{h}^{\alpha \beta}=\frac{1}{12} \eta^{\mu \nu} c_{\mu \nu}^{\alpha \beta}
$$

Lemma 2 The coefficients $\hat{r}^{\alpha \beta}$ are symmetric w.r.t. $\alpha$ and $\beta$, i.e.,

$$
\hat{r}^{\alpha \beta}=\hat{r}^{\beta \alpha} .
$$

The proofs of Lemma 1 and Lemma 8 are similar to those of Lemma 5 and Lemma 6 which will be given below, however, the computation is much more simple, so we omit the proofs here.

Lemma 3 For the Hamiltonians $H_{\alpha, p}$ defined in (2.45) the following identity holds true:

$$
H_{\alpha, p}=\int \theta_{\alpha, p+1}(v(X)) d X=\int \theta_{\alpha, p+1}(t(X)) d X+\varepsilon^{2} \delta H_{\alpha, p}^{\prime}+\mathcal{O}\left(\varepsilon^{4}\right),
$$

where $\delta H_{\alpha, p}^{\prime}$ are defined by (3.10) and (3.11). 
Proof By using (2.40) we have

$$
\begin{aligned}
H_{\alpha, p}= & \int \theta_{\alpha, p+1}(v(X)) d X \\
= & \int \theta_{\alpha, p+1}(t(X)) d X-\varepsilon^{2} \int \frac{\partial \theta_{\alpha, p+1}(t(X))}{\partial t^{\mu}} w^{\mu} d X+\mathcal{O}\left(\varepsilon^{4}\right) \\
= & \int \theta_{\alpha, p+1}(t(X)) d X+\varepsilon^{2} \int \frac{\partial^{2} \theta_{\alpha, p+1}(t(X))}{\partial t^{\mu} \partial t^{\nu}} t_{X}^{\nu} \frac{\partial F^{(1)}\left(t(X), t_{X}(X)\right)}{\partial T_{\mu, 0}} d X+\mathcal{O}\left(\varepsilon^{4}\right) \\
= & \int \theta_{\alpha, p+1}(t(X)) d X+\varepsilon^{2} \int c_{\mu \nu}^{\gamma} \frac{\partial \theta_{\alpha, p}}{\partial t^{\gamma}} t_{X}^{\nu}\left(F_{t^{\sigma}}^{(1)} c_{\rho}^{\sigma \mu} t_{X}^{\rho}+F_{t_{X}^{\sigma}}^{(1)} c_{\rho \xi}^{\sigma \mu} t_{X}^{\rho} t_{X}^{\xi}\right. \\
& \left.+F_{t_{X}^{\sigma}}^{(1)} c_{\rho}^{\sigma \mu} t_{X X}^{\rho}\right) d X+\mathcal{O}\left(\varepsilon^{4}\right) .
\end{aligned}
$$

Now formulas (4.7), (4.17), (4.20) and (4.21) amount to

$$
\begin{aligned}
c_{\mu \nu}^{\gamma} & \frac{\partial \theta_{\alpha, p}}{\partial t^{\gamma}} t_{X}^{\nu}\left(F_{t^{\sigma}}^{(1)} c_{\rho}^{\sigma \mu} t_{X}^{\rho}+F_{t_{X}^{\sigma}}^{(1)} c_{\rho \xi}^{\sigma \mu} t_{X}^{\rho} t_{X}^{\xi}\right) \\
= & \frac{1}{24} \gamma_{i j} \frac{\partial \theta_{\alpha, p}}{\partial t^{\gamma}}\left(2 \sigma_{i}^{2} \frac{\psi_{i}^{\gamma} \psi_{j 1}}{\psi_{i 1}^{4}}+\sigma_{j}^{2} \frac{\psi_{j}^{\gamma}}{\psi_{i 1} \psi_{j 1}^{2}}+\sigma_{j}^{2} \frac{\psi_{i}^{\gamma}}{\psi_{i 1}^{2} \psi_{j 1}}\right. \\
& \left.-3 \sigma_{i} \sigma_{j} \frac{\psi_{i}^{\gamma}}{\psi_{i 1}^{3}}-\sigma_{i} \sigma_{j} \frac{\psi_{j}^{\gamma}}{\psi_{i 1}^{2} \psi_{j 1}}\right)
\end{aligned}
$$

and

$$
\begin{aligned}
& \int c_{\mu \nu}^{\gamma} \frac{\partial \theta_{\alpha, p}}{\partial t^{\gamma}} t_{X}^{\nu} F_{t_{X}^{\sigma}}^{(1)} c_{\rho}^{\sigma \mu} t_{X X}^{\rho} d X \\
& =\frac{1}{24} \int \frac{\partial \theta_{\alpha, p}}{\partial t^{\gamma}} \frac{\psi_{j}^{\gamma} \psi_{j \mu} \psi_{j \nu}}{\psi_{j 1}} t_{X}^{\nu} \frac{\psi_{i \sigma}}{\sigma_{i}} \frac{\psi_{k}^{\sigma} \psi_{k}^{\mu} \psi_{k \rho}}{\psi_{k 1}} t_{X X}^{\rho} \\
& =\frac{1}{24} \int \frac{\partial \theta_{\alpha, p}}{\partial t^{\gamma}} \frac{\psi_{j}^{\gamma} \psi_{j \mu} \sigma_{j}}{\psi_{j 1}} \frac{\psi_{i \sigma}}{\sigma_{i}} \frac{\psi_{k}^{\sigma} \psi_{k}^{\mu} \psi_{k \rho}}{\psi_{k 1}} t_{X X}^{\rho} \\
& =\frac{1}{24} \int \frac{\partial \theta_{\alpha, p}}{\partial t^{\gamma}} \frac{\psi_{j}^{\gamma} \psi_{j \rho}}{\psi_{j 1}^{2}} t_{X X}^{\rho} d X=-\frac{1}{24} \int \frac{\partial}{\partial t^{\nu}}\left(\frac{\partial \theta_{\alpha, p}}{\partial t^{\gamma}} \frac{\psi_{j}^{\gamma} \psi_{j \rho}}{\psi_{j 1}^{2}}\right) t_{X}^{\rho} t_{X}^{\nu} d X
\end{aligned}
$$

So the Hamiltonians $H^{\alpha, p}$ can be expressed in the form (5.6), (3.10) with

$$
\begin{gathered}
\chi_{\alpha, p+1 ; \mu \nu}=\frac{\gamma_{i j}}{24} \frac{\partial \theta_{\alpha, p}}{\partial t^{\gamma}}\left(\frac{2 \psi_{i \mu} \psi_{i \nu} \psi_{i}^{\gamma} \psi_{j 1}}{\psi_{i 1}^{4}}+\frac{\psi_{j \mu} \psi_{j \nu} \psi_{j}^{\gamma}}{\psi_{i 1} \psi_{j 1}^{2}}+\frac{\psi_{j \mu} \psi_{j \nu} \psi_{i}^{\gamma}}{\psi_{i 1}^{2} \psi_{j 1}}\right. \\
-\frac{3 \psi_{i \mu} \psi_{j \nu} \psi_{i}^{\gamma}}{2 \psi_{i 1}^{3}}-\frac{3 \psi_{i \nu} \psi_{j \mu} \psi_{i}^{\gamma}}{2 \psi_{i 1}^{3}}-\frac{\psi_{i \mu} \psi_{j \nu} \psi_{j}^{\gamma}}{2 \psi_{i 1}^{2} \psi_{j 1}} \\
\left.-\frac{\psi_{i \nu} \psi_{j \mu} \psi_{j}^{\gamma}}{2 \psi_{i 1}^{2} \psi_{j 1}}\right)-\frac{1}{48} \frac{\partial}{\partial t^{\nu}}\left(\frac{\partial \theta_{\alpha, p}}{\partial t^{\gamma}} \frac{\psi_{j}^{\gamma} \psi_{j \mu}}{\psi_{j 1}^{2}}\right)-\frac{1}{48} \frac{\partial}{\partial t^{\mu}}\left(\frac{\partial \theta_{\alpha, p}}{\partial t^{\gamma}} \frac{\psi_{j}^{\gamma} \psi_{j \nu}}{\psi_{j 1}^{2}}\right) \\
=\frac{1}{24} w_{\mu \nu}^{\gamma} \frac{\partial \theta^{\alpha, p-1}}{\partial t^{\gamma}}-\frac{1}{24} c_{\xi \zeta}^{\gamma} c_{\nu}^{\xi \sigma} c_{\sigma \mu}^{\zeta} \frac{\partial \theta_{\alpha, p-1}}{\partial t^{\gamma}}, \quad p \geq-1,
\end{gathered}
$$

where $\theta_{\alpha,-2}=\theta_{\alpha,-1}=0$ and $w_{\mu \nu}^{\alpha}$ are defined in (3.12). Lemma is proved. 
Proof of Theorem 1 We first prove the formula (3.9) for the first Poisson bracket. From Lemma 1 and Lemma 2 we already know the expression of the coefficients $\hat{h}^{\alpha \beta}$ and the anti-symmetric part of the coefficients $\hat{r}^{\alpha \beta}$ in the formula (5.3). Now from the fact that the Casimirs of the first Poisson bracket $\int v^{\gamma}(X) d X$ have the expression

$$
\int v^{\gamma}(X) d X=\int t^{\gamma}(X) d X+\mathcal{O}\left(\varepsilon^{4}\right)
$$

we see that

$$
\hat{p}^{\alpha \beta}=0
$$

in the formula (5.3). So the anti-symmetry condition of the first Poisson bracket gives us the following relations:

$$
\begin{aligned}
& \hat{r}^{\alpha \beta}+\hat{r}^{\beta \alpha}=3 \partial_{X} \hat{h}^{\alpha \beta}, \\
& \hat{f}^{\beta \alpha}-\hat{f}^{\alpha \beta}+2 \partial_{X} \hat{r}^{\alpha \beta}=3 \partial_{X}^{2} \hat{h}^{\alpha \beta}, \\
& \partial_{X} \hat{f}^{\alpha \beta}+\partial_{X}^{3} \hat{h}^{\alpha \beta}-\partial_{X}^{2} \hat{r}^{\alpha \beta}=0 .
\end{aligned}
$$

Identity (5.10) together with (5.5) gives us the expression for $\hat{r}^{\alpha \beta}$, while from the identity (5.12) it follows that

$$
\hat{f}^{\alpha \beta}=\partial_{X} \hat{r}^{\alpha \beta}-\partial_{X}^{2} \hat{h}^{\alpha \beta}
$$

there is no integration constant because $\hat{f}^{\alpha \beta}$ must depend on $t_{X}^{\gamma}$ or $t_{X X}^{\gamma}$. So we get the expression for the coefficients $\hat{f}^{\alpha \beta}$ and complete the proof of formula (3.9). The remaining part of the Theorem follows from Lemma 3. Theorem is proved.

We now proceed to prove Theorem 2. So we still assume here that $G=0$ in the formulae (3.2), (3.3). Doing the same infinitesimal Bäcklund tranform (3.2) (with $G(t)=0)$ we obtain

$$
\begin{aligned}
& \left\{t^{\alpha}(X), t^{\beta}(Y)\right\}_{2}^{\prime}=g^{\alpha \beta}(t(X)) \delta^{\prime}(X-Y)+\Gamma_{\gamma}^{\alpha \beta}(t(X)) t_{X}^{\gamma} \delta(X-Y) \\
& -\varepsilon^{2}\left[\frac{\partial g^{\alpha \beta}(t(X))}{\partial t^{\gamma}} w^{\gamma}(t(X)) \delta^{\prime}(X-Y)+\frac{\partial \Gamma_{\gamma}^{\alpha \beta}(t(X))}{\partial t^{\mu}} w^{\mu}(t(X)) t_{X}^{\gamma} \delta(X-Y)\right. \\
& +\Gamma_{\gamma}^{\alpha \beta}(t(X))\left(\partial_{X} w^{\gamma}(t(X)) \delta(X-Y)\right) \\
& +\left(\frac{\partial w^{\beta}(t(Y))}{\partial t^{\gamma}}+\frac{\partial w^{\beta}(t(Y))}{\partial t_{Y}^{\gamma}} \partial_{Y}+\frac{\partial w^{\beta}(t(Y))}{\partial t_{Y Y}^{\gamma}} \partial_{Y}^{2}+\frac{\partial w^{\beta}(t(Y))}{\partial t_{Y Y Y}^{\gamma}} \partial_{Y}^{3}\right) \\
& \quad \times\left(g^{\alpha \gamma}(t(X)) \delta^{\prime}(X-Y)+\Gamma_{\mu}^{\alpha \gamma}(t(X)) t_{X}^{\mu} \delta(X-Y)\right) \\
& +\left(\frac{\partial w^{\alpha}(t(X))}{\partial t^{\gamma}}+\frac{\partial w^{\alpha}(t(X))}{\partial t_{X}^{\gamma}} \partial_{X}+\frac{\partial w^{\alpha}(t(X))}{\partial t_{X X}^{\gamma}} \partial_{X}^{2}+\frac{\partial w^{\alpha}(t(X))}{\partial t_{X X X}^{\gamma}} \partial_{X}^{3}\right) \\
& \left.\times\left(g^{\gamma \beta}(t(X)) \delta^{\prime}(X-Y)+\Gamma_{\mu}^{\gamma \beta}(t(X)) t_{X}^{\mu} \delta(X-Y)\right)+\mathcal{O}\left(\varepsilon^{4}\right)\right]
\end{aligned}
$$

In the Poisson bracket $\left\{t^{\alpha}(X), t^{\beta}(Y)\right\}_{2}$, the coefficient of $\varepsilon^{2} \delta^{(4)}(X-Y)$ is equal to

$$
-\frac{\partial w^{\beta}(t(X))}{\partial t_{X X X}^{\gamma}} g^{\gamma \alpha}+\frac{\partial w^{\alpha}(t(X))}{\partial t_{X X X}^{\gamma}} g^{\gamma \beta}=-F_{t_{X}^{\mu}}^{(1)} c_{\gamma}^{\mu \beta} g^{\gamma \alpha}+F_{t_{X}^{\mu}}^{(1)} c_{\gamma}^{\mu \alpha} g^{\gamma \beta}=0,
$$


the last equality above is due to the associativity equation

$$
c_{\gamma}^{\mu \beta} c_{\nu}^{\gamma \alpha}=c_{\gamma}^{\mu \alpha} c_{\nu}^{\gamma \beta}
$$

and the definition (2.22) of the intersection form.

The coefficient of $\varepsilon^{2} \delta^{(3)}(X-Y)$ is equal to

$$
\begin{aligned}
h^{\alpha \beta}= & 2 F_{t^{\mu}}^{(1)} c_{\gamma}^{\alpha \mu} g^{\gamma \beta}+3 F_{t_{X}^{\mu}}^{(1)} c_{\gamma \xi}^{\mu \alpha} g^{\beta \gamma} t_{X}^{\xi}-F_{t_{X}^{\mu}}^{(1)} c_{\gamma \xi}^{\beta \mu} g^{\alpha \gamma} t_{X}^{\xi}-2 F_{t_{X}^{\mu} t^{\xi}}^{(1)} c_{\gamma}^{\alpha \mu} g^{\gamma \beta} t_{X}^{\xi} \\
& +F_{t_{X}^{\gamma} t^{\mu}}^{(1)}\left(c_{\xi}^{\alpha \mu} g^{\gamma \beta}+c_{\xi}^{\beta \mu} g^{\gamma \alpha}\right) t_{X}^{\xi}+F_{t_{X}^{\mu} t_{X}^{\gamma}}^{(1)}\left(c_{\nu \xi}^{\mu \alpha} g^{\gamma \beta}+c_{\nu \xi}^{\mu \beta} g^{\gamma \alpha}\right) t_{X}^{\nu} t_{X}^{\xi} \\
& +3 F_{t_{X}^{\mu}}^{(1)} c_{\gamma}^{\alpha \mu} \frac{\partial g^{\gamma \beta}}{\partial t^{\xi}} t_{X}^{\xi}-F_{t_{X}^{\mu}}^{(1)} c_{\gamma}^{\beta \mu} \Gamma_{\xi}^{\alpha \gamma} t_{X}^{\xi}+F_{t_{X}^{\mu}}^{(1)} c_{\gamma}^{\alpha \mu} \Gamma_{\xi}^{\gamma \beta} t_{X}^{\xi}+S^{\alpha \beta}
\end{aligned}
$$

where

$$
\begin{aligned}
S^{\alpha \beta}= & \left(F_{t_{X}^{\mu} t_{X}^{\xi}}^{(1)} c_{\gamma}^{\beta \mu} g^{\alpha \gamma}+F_{t_{X}^{\mu} t_{X}^{\xi}}^{(1)} c_{\gamma}^{\alpha \mu} g^{\beta \gamma}+F_{t_{X}^{\mu} t_{X}^{\gamma}}^{(1)} c_{\xi}^{\beta \mu} g^{\alpha \gamma}+F_{t_{X}^{\mu} t_{X}^{\gamma}}^{(1)} c_{\xi}^{\alpha \mu} g^{\beta \gamma}\right. \\
& \left.-4 F_{t_{X}^{\mu} t_{X}^{\xi}}^{(1)} c_{\gamma}^{\beta \mu} g^{\alpha \gamma}\right) t_{X X}^{\xi} \\
= & \left(F_{t_{X}^{\mu} t_{X}^{\gamma}}^{(1)} c_{\xi}^{\beta \mu} g^{\alpha \gamma}+F_{t_{X}^{\mu} t_{X}^{\gamma}}^{(1)} c_{\xi}^{\alpha \mu} g^{\beta \gamma}-F_{t_{X}^{\mu} t_{X}^{\xi}}^{(1)} c_{\gamma}^{\beta \mu} g^{\alpha \gamma}-F_{t_{X}^{\mu} t_{X}^{\xi}}^{(1)} c_{\gamma}^{\alpha \mu} g^{\beta \gamma}\right) t_{X X}^{\xi}
\end{aligned}
$$

Lemma $4 \quad S^{\alpha \beta}=0$.

Proof By using (2.22), (4.7) and (4.18) we have the identity

$$
F_{t_{X}^{\mu}}^{(1)} t_{X}^{\gamma} c_{\nu}^{\beta \mu} g^{\alpha \gamma}=F_{t_{X}^{\mu}}^{(1)} t_{X}^{\nu} c_{\gamma}^{\alpha \mu} g^{\beta \gamma},
$$

since both sides of the above identity are equal to

$$
-\frac{1}{24} \sum_{i} \frac{E^{\xi} \psi_{i}^{\alpha} \psi_{i}^{\beta} \psi_{i \nu} \psi_{i \xi}}{\sigma_{i}^{2} \psi_{i 1}^{2}}
$$

The lemma follows from the above identity immediately. Lemma is proved.

Lemma 5 The coefficients $h^{\alpha \beta}$ defined in (5.17) have the expression

$$
h^{\alpha \beta}=\frac{1}{12}\left(\frac{\partial}{\partial t^{\nu}}\left(g^{\mu \nu} c_{\mu}^{\alpha \beta}\right)+\frac{1}{2} c_{\nu}^{\mu \nu} c_{\mu}^{\alpha \beta}\right) .
$$

Proof Let's rewrite $24 h^{\alpha \beta}$ as the sum of $A^{\alpha \beta}$ and $B^{\alpha \beta}$, where

$$
\begin{aligned}
A^{\alpha \beta}= & 24\left(2 F_{t^{\mu}}^{(1)} c_{\gamma}^{\alpha \mu} g^{\gamma \beta}+3 F_{t_{X}^{\mu}}^{(1)} c_{\gamma \xi}^{\mu \alpha} g^{\beta \gamma} t_{X}^{\xi}-F_{t_{X}^{\mu}}^{(1)} c_{\gamma \xi}^{\beta \mu} g^{\alpha \gamma} t_{X}^{\xi}-2 F_{t_{X}^{\mu} t^{\xi}}^{(1)} c_{\gamma}^{\alpha \mu} g^{\gamma \beta} t_{X}^{\xi}\right. \\
& \left.+F_{t_{X}^{\gamma} t^{\mu}}^{(1)}\left(c_{\xi}^{\alpha \mu} g^{\gamma \beta}+c_{\xi}^{\beta \mu} g^{\gamma \alpha}\right) t_{X}^{\xi}+F_{t_{X}^{\mu} t_{X}^{\gamma}}^{(1)}\left(c_{\nu \xi}^{\mu \alpha} g^{\gamma \beta}+c_{\nu \xi}^{\mu \beta} g^{\gamma \alpha}\right) t_{X}^{\nu} t_{X}^{\xi}\right),
\end{aligned}
$$

and

$$
B^{\alpha \beta}=24\left(3 F_{t_{X}^{\mu}}^{(1)} c_{\gamma}^{\alpha \mu} \frac{\partial g^{\gamma \beta}}{\partial t^{\xi}} t_{X}^{\xi}-F_{t_{X}^{\mu}}^{(1)} c_{\gamma}^{\beta \mu} \Gamma_{\xi}^{\alpha \gamma} t_{X}^{\xi}+F_{t_{X}^{\mu}}^{(1)} c_{\gamma}^{\alpha \mu} \Gamma_{\xi}^{\gamma \beta} t_{X}^{\xi}\right)
$$


By using the formulas given in Section 4 we have

$$
\begin{aligned}
& A^{\alpha \beta}=\frac{\gamma_{i j} \sigma_{j}}{\sigma_{i} \psi_{i 1} \psi_{j 1}}\left(3 \psi_{j \gamma} \psi_{i}^{\alpha} g^{\gamma \beta}-3 \psi_{i \gamma} \psi_{j}^{\beta} g^{\gamma \alpha}+\psi_{i \gamma} \psi_{j}^{\alpha} g^{\gamma \beta}-\psi_{j \gamma} \psi_{i}^{\beta} g^{\gamma \alpha}\right) \\
& +2 \gamma_{i j}\left(\frac{\psi_{i}^{\alpha} \psi_{i \gamma} g^{\gamma \beta}}{\psi_{i 1} \psi_{j 1}}-\frac{\psi_{j}^{\alpha} \psi_{i \gamma} g^{\gamma \beta}}{\psi_{i 1}^{2}}-2 \frac{\psi_{i}^{\alpha} \psi_{j \gamma} g^{\gamma \beta}}{\psi_{i 1}^{2}}+\frac{\psi_{j}^{\beta} \psi_{i \gamma} g^{\gamma \alpha}}{\psi_{i 1}^{2}}\right. \\
& \left.+\frac{\psi_{i}^{\alpha} \psi_{i \gamma} \psi_{j 1} g^{\gamma \beta}}{\psi_{i 1}^{3}}\right)
\end{aligned}
$$

For $B^{\alpha \beta}$, by using the formulas given in Section 4 and the following formulas

$$
\Gamma_{\gamma}^{\alpha \beta}=\left(\frac{1+d}{2}-q_{\beta}\right) c_{\gamma}^{\alpha \beta}, \quad \frac{\partial g^{\alpha \beta}}{\partial t^{\gamma}}=\Gamma_{\gamma}^{\alpha \beta}+\Gamma_{\gamma}^{\beta \alpha},
$$

we obtain

$$
\begin{aligned}
B^{\alpha \beta} & =24 F_{t_{X}^{\mu}}^{(1)}\left(\left(3+3 d-4 q_{\beta}\right) c_{\gamma}^{\alpha \mu} c_{\xi}^{\gamma \beta}-3 q_{\gamma} c_{\gamma}^{\alpha \mu} c_{\xi}^{\gamma \beta}+q_{\gamma} c_{\gamma}^{\beta \mu} c_{\xi}^{\gamma \alpha}\right) t_{X}^{\xi} \\
& =\left(3+3 d-4 q_{\beta}\right) \frac{\psi_{i}^{\alpha} \psi_{i}^{\beta}}{\psi_{i 1}^{2}}+\frac{\sigma_{j}}{\sigma_{i}} \frac{q_{\gamma} \psi_{i \gamma} \psi_{j}^{\gamma}}{\psi_{i 1} \psi_{j 1}}\left(\psi_{j}^{\alpha} \psi_{i}^{\beta}-3 \psi_{j}^{\beta} \psi_{i}^{\alpha}\right) \\
& =\left(3+3 d-4 q_{\beta}\right) \frac{\psi_{i}^{\alpha} \psi_{i}^{\beta}}{\psi_{i 1}^{2}}+\frac{\sigma_{j}}{\sigma_{i}}\left(\left(u_{j}-u_{i}\right) \gamma_{i j}+\frac{d}{2} \delta_{i j}\right) \frac{1}{\psi_{i 1} \psi_{j 1}}\left(\psi_{j}^{\alpha} \psi_{i}^{\beta}-3 \psi_{j}^{\beta} \psi_{i}^{\alpha}\right) \\
& =\left(3+2 d-4 q_{\beta}\right) \frac{\psi_{i}^{\alpha} \psi_{i}^{\beta}}{\psi_{i 1}^{2}}+\frac{\sigma_{j}}{\sigma_{i}} \frac{\left(u_{j}-u_{i}\right) \gamma_{i j}}{\psi_{i 1} \psi_{j 1}}\left(\psi_{j}^{\alpha} \psi_{i}^{\beta}-3 \psi_{j}^{\beta} \psi_{i}^{\alpha}\right),
\end{aligned}
$$

above we have used formula (4.28). From formula (4.27) it follows that

$$
\begin{aligned}
& \quad 24 h^{\alpha \beta}=A^{\alpha \beta}+B^{\alpha \beta} \\
& =\quad 2 \gamma_{i j}\left(\frac{\psi_{i}^{\alpha} \psi_{i \gamma} g^{\gamma \beta}}{\psi_{i 1} \psi_{j 1}}-\frac{\psi_{j}^{\alpha} \psi_{i \gamma} g^{\gamma \beta}}{\psi_{i 1}^{2}}-2 \frac{\psi_{i}^{\alpha} \psi_{j \gamma} g^{\gamma \beta}}{\psi_{i 1}^{2}}+\frac{\psi_{j}^{\beta} \psi_{i \gamma} g^{\gamma \alpha}}{\psi_{i 1}^{2}}+\frac{\psi_{i}^{\alpha} \psi_{i \gamma} \psi_{j 1} g^{\gamma \beta}}{\psi_{i 1}^{3}}\right) \\
& +\left(3+2 d-4 q_{\beta}\right) \frac{\psi_{i}^{\alpha} \psi_{i}^{\beta}}{\psi_{i 1}^{2}} .
\end{aligned}
$$

On the other hand, for the right-hand side of (5.20) we have

$$
\begin{array}{ll} 
& 2 \frac{\partial}{\partial t^{\nu}}\left(g^{\mu \nu} c_{\mu}^{\alpha \beta}\right)+c_{\nu}^{\mu \nu} c_{\mu}^{\alpha \beta} \\
= & -2 \frac{\partial}{\partial t^{\nu}}\left(g^{\mu \nu} c_{\mu}^{\alpha \beta}\right)+4 \frac{\partial}{\partial t^{\nu}}\left(g^{\mu \beta} c_{\mu}^{\alpha \nu}\right)+c_{\nu}^{\mu \nu} c_{\mu}^{\alpha \beta} \\
=\quad & -2\left(1+d-q_{\mu}-q_{\nu}\right) c_{\nu}^{\mu \nu} c_{\mu}^{\alpha \beta}+4\left(1+d-q_{\mu}-q_{\beta}\right) c_{\nu}^{\mu \beta} c_{\mu}^{\alpha \nu} \\
& +c_{\nu}^{\mu \nu} c_{\mu}^{\alpha \beta}-2 g^{\mu \nu} c_{\mu \nu}^{\alpha \beta}+4 g^{\mu \beta} c_{\mu \nu}^{\alpha \nu} \\
= & \left(3+2 d-4 q_{\beta}\right) \frac{\psi_{i}^{\alpha} \psi_{i}^{\beta}}{\psi_{i 1}^{2}}-2 \gamma_{i j}\left(u_{i}-u_{j}\right) \frac{\psi_{i}^{\alpha} \psi_{i}^{\beta}}{\psi_{i 1} \psi_{j 1}}
\end{array}
$$




$$
\begin{aligned}
& -2 \gamma_{i j}\left(\frac{u_{j} \psi_{i}^{\alpha} \psi_{i}^{\beta}}{\psi_{i 1} \psi_{j 1}}-\frac{u_{i} \psi_{i}^{\beta} \psi_{j}^{\alpha}}{\psi_{i 1}^{2}}-\frac{u_{i} \psi_{i}^{\alpha} \psi_{j}^{\beta}}{\psi_{i 1}^{2}}+\frac{u_{i} \psi_{i}^{\alpha} \psi_{i}^{\beta} \psi_{j 1}}{\psi_{i 1}^{3}}\right) \\
& +4 \gamma_{i j}\left(\frac{\psi_{i}^{\alpha} \psi_{i \gamma}}{\psi_{i 1} \psi_{j 1}}-\frac{\psi_{j}^{\alpha} \psi_{i \gamma}}{\psi_{i 1}^{2}}-\frac{\psi_{i}^{\alpha} \psi_{j \gamma}}{\psi_{i 1}^{2}}+\frac{\psi_{i}^{\alpha} \psi_{i \gamma} \psi_{j 1}}{\psi_{i 1}^{3}}\right) g^{\gamma \beta} \\
= & 2 \gamma_{i j}\left(\frac{\psi_{i}^{\alpha} \psi_{i \gamma} g^{\gamma \beta}}{\psi_{i 1} \psi_{j 1}}-\frac{\psi_{j}^{\alpha} \psi_{i \gamma} g^{\gamma \beta}}{\psi_{i 1}^{2}}-2 \frac{\psi_{i}^{\alpha} \psi_{j \gamma} g^{\gamma \beta}}{\psi_{i 1}^{2}}+\frac{\psi_{j}^{\beta} \psi_{i \gamma} g^{\gamma \alpha}}{\psi_{i 1}^{2}}+\frac{\psi_{i}^{\alpha} \psi_{i \gamma} \psi_{j 1} g^{\gamma \beta}}{\psi_{i 1}^{3}}\right) \\
& +\left(3+2 d-4 q_{\beta}\right) \frac{\psi_{i}^{\alpha} \psi_{i}^{\beta}}{\psi_{i 1}^{2}} \\
= & 24 h^{\alpha \beta} .
\end{aligned}
$$

Lemma is proved.

Knowing $h^{\alpha \beta}(t)$ we can compute the symmetrized coefficient in front of $\delta^{\prime \prime}(X-Y)$ using the skew-symmetry condition

$$
r_{\gamma}^{\alpha \beta}(t)+r_{\gamma}^{\beta \alpha}(t)=3 \partial_{\gamma} h^{\alpha \beta}
$$

The antisymmetrization of the same coefficients is given by the following:

Lemma 6 Let's denote $\tilde{r}^{\alpha \beta}$ the coefficients before $\varepsilon^{2} \delta^{\prime \prime}(X-Y)$ in the second Poisson bracket $\left\{t^{\alpha}(X), t^{\beta}(Y)\right\}_{2}^{\prime}$ of (5.14), then the following identity holds true:

$$
\tilde{r}^{\alpha \beta}-\tilde{r}^{\beta \alpha}=\frac{1}{24}\left(d+3-2 q_{\beta}\right) c_{\gamma}^{\alpha \nu} c_{\nu \mu}^{\beta \mu} t_{X}^{\gamma}-\frac{1}{24}\left(d+3-2 q_{\alpha}\right) c_{\gamma}^{\beta \nu} c_{\nu \mu}^{\alpha \mu} t_{X}^{\gamma} .
$$

Proof From the expression (5.14) we have

$$
\begin{aligned}
\tilde{r}^{\alpha \beta} & =g^{\gamma \beta} \frac{\partial w^{\alpha}}{\partial t_{X}^{\gamma}}-g^{\gamma \alpha} \frac{\partial w^{\beta}}{\partial t_{X}^{\gamma}}+3 g^{\gamma \alpha} \partial_{X}\left(\frac{\partial w^{\beta}}{\partial t_{X X}^{\gamma}}\right)-6 g^{\gamma \alpha} \partial_{X}^{2}\left(\frac{\partial w^{\beta}}{\partial t_{X X X}^{\gamma}}\right) \\
& +3 \Gamma_{\mu}^{\gamma \beta} \frac{\partial w^{\alpha}}{\partial t_{X X}^{\gamma}} t_{X}^{\mu}+2 \Gamma_{\mu}^{\beta \gamma} \frac{\partial w^{\alpha}}{\partial t_{X X}^{\gamma}} t_{X}^{\mu}+\Gamma_{\mu}^{\alpha \gamma} \frac{\partial w^{\beta}}{\partial t_{X X}^{\gamma}} t_{X}^{\mu} \\
& -3 \Gamma_{\mu}^{\alpha \gamma} \partial_{X}\left(\frac{\partial w^{\beta}}{\partial t_{X X X}^{\gamma}}\right) t_{X}^{\mu}+3 \frac{\partial_{X}^{2} g^{\gamma \beta}}{\partial X^{2}} \frac{\partial w^{\alpha}}{\partial t_{X X X}^{\gamma}}+3 \frac{\partial\left(\Gamma_{\mu}^{\gamma \beta} t_{X}^{\mu}\right)}{\partial X} \frac{\partial w^{\alpha}}{\partial t_{X X X}^{\gamma}} .
\end{aligned}
$$

By using the formulas given in Section 2 we get, through a long calculation, the following:

$$
\begin{gathered}
\tilde{r}^{\alpha \beta}-\tilde{r}^{\beta \alpha}=2 g^{\gamma \beta} \frac{\partial w^{\alpha}}{\partial t_{X}^{\gamma}}-3 g^{\gamma \beta} \partial_{X}\left(\frac{\partial w^{\alpha}}{\partial t_{X X}^{\gamma}}\right)+6 g^{\gamma \beta} \partial_{X}^{2}\left(\frac{\partial w^{\alpha}}{\partial t_{X X X}^{\gamma}}\right) \\
+3 \Gamma_{\mu}^{\gamma \beta} \frac{\partial w^{\alpha}}{\partial t_{X X}^{\gamma}} t_{X}^{\mu}+\Gamma_{\mu}^{\beta \gamma} \frac{\partial w^{\alpha}}{\partial t_{X X}^{\gamma}} t_{X}^{\mu}+3 \Gamma_{\mu}^{\beta \gamma} \partial_{X}\left(\frac{\partial w^{\alpha}}{\partial t_{X X X}^{\gamma}}\right) t_{X}^{\mu} \\
+3 \frac{\partial^{2} g^{\gamma \beta}}{\partial X^{2}} \frac{\partial w^{\alpha}}{\partial t_{X X X}^{\gamma}}+3 \frac{\partial\left(\Gamma_{\mu}^{\gamma \beta} t_{X}^{\mu}\right)}{\partial X} \frac{\partial w^{\alpha}}{\partial t_{X X X}^{\gamma}}
\end{gathered}
$$


- (the precedent sum with $\alpha$ and $\beta$ changed)

$$
\begin{aligned}
= & 2 g^{\gamma \beta} \partial_{X}\left(\frac{\partial}{\partial t_{X}^{\gamma}} \frac{\partial F^{(1)}}{\partial T_{\alpha, 0}}\right)+2 g^{\gamma \beta}\left(\frac{\partial}{\partial t^{\gamma}} \frac{\partial F^{(1)}}{\partial T_{\alpha, 0}}\right)-3 g^{\gamma \beta} \partial_{X}\left(\frac{\partial w^{\alpha}}{\partial t_{X X}^{\gamma}}\right) \\
& +6 g^{\gamma \beta} \partial_{X}^{2}\left(\frac{\partial w^{\alpha}}{\partial t_{X X X}^{\gamma}}\right)+3 \Gamma_{\mu}^{\gamma \beta} \frac{\partial w^{\alpha}}{\partial t_{X X}^{\gamma}} t_{X}^{\mu}+\Gamma_{\mu}^{\beta \gamma} \frac{\partial w^{\alpha}}{\partial t_{X X}^{\gamma}} t_{X}^{\mu} \\
& +3 \Gamma_{\mu}^{\beta \gamma} \partial_{X}\left(\frac{\partial w^{\alpha}}{\partial t_{X X X}^{\gamma}}\right) t_{X}^{\mu}+3 \frac{\partial^{2} g^{\gamma \beta}}{\partial X^{2}} \frac{\partial w^{\alpha}}{\partial t_{X X X}^{\gamma}}+3 \frac{\partial\left(\Gamma_{\mu}^{\gamma \beta} t_{X}^{\mu}\right)}{\partial X} \frac{\partial w^{\alpha}}{\partial t_{X X X}^{\gamma}}
\end{aligned}
$$

- (the precedent sum with $\alpha$ and $\beta$ exchanged)

$$
\begin{aligned}
= & 2 g^{\gamma \beta}\left(F_{t^{\mu} t^{\gamma}}^{(1)} c_{\nu}^{\alpha \mu} t_{X}^{\nu}+F_{t_{X}^{\mu} t_{\gamma}}^{(1)} c_{\nu_{0}}^{\alpha \mu} t_{X}^{\nu} t_{X}^{\rho}+F_{t_{X}^{\mu} t^{\gamma}}^{(1)} c_{\nu}^{\alpha \mu} t_{X X}^{\nu}\right) \\
& -g^{\gamma \beta} \partial_{X}\left(F_{t^{\mu}}^{(1)} t_{X}^{\gamma} c_{\nu}^{\alpha \mu} t_{X}^{\nu}+F_{t_{X}^{\mu} t_{X}^{\gamma}}^{(1)} c_{\nu \sigma}^{\alpha \mu} t_{X}^{\nu} t_{X}^{\sigma}\right) \\
& +g^{\gamma \beta} \partial_{X}\left(F_{t_{X}^{\mu}}^{(1)} c_{\gamma \nu}^{\alpha \mu} t_{X}^{\nu}+3 F_{t_{X}^{\mu} t^{\nu}}^{(1)} c_{\gamma}^{\alpha \mu} t_{X}^{\nu}+3 F_{t_{X}^{\mu} t_{X}^{\nu}}^{(1)} c_{\gamma}^{\alpha \mu} t_{X X}^{\nu}-F_{t_{X}^{\mu} t_{X}^{\gamma} c_{\nu}^{\alpha \mu} t_{X X}^{\nu}}^{\nu}\right. \\
& \left.-F_{t^{\mu}}^{(1)} c_{\gamma}^{\alpha \mu}\right)+2 g^{\gamma \beta}\left(F_{t^{\mu}}^{(1)} c_{\gamma \nu}^{\alpha \mu} t_{X}^{\nu}+F_{t_{X}^{\mu}}^{(1)} c_{\nu \rho \gamma}^{\alpha \mu} t_{X}^{\nu} t_{X}^{\rho}+F_{t_{X}^{\prime \mu}}^{(1)} c_{\nu \gamma}^{\alpha \mu} t_{X X}^{\nu}\right) \\
& +3 \Gamma_{\mu}^{\gamma \beta} \frac{\partial w^{\alpha}}{\partial t_{X X}^{\gamma}} t_{X}^{\mu}+\Gamma_{\mu}^{\beta \gamma} \frac{\partial w^{\alpha}}{\partial t_{X X}^{\gamma}} t_{X}^{\mu}+3 \Gamma_{\mu}^{\beta \gamma} \partial_{X}\left(\frac{\partial w^{\alpha}}{\partial t_{X X X}^{\gamma}}\right) t_{X}^{\mu} \\
& +3 \frac{\partial^{2} g^{\gamma \beta}}{\partial X^{2}} \frac{\partial w^{\alpha}}{\partial t_{X X X}^{\gamma}}+3 \frac{\partial\left(\Gamma_{\mu}^{\gamma \beta} t_{X}^{\mu}\right)}{\partial X} \frac{\partial w^{\alpha}}{\partial t_{X X X}^{\gamma}}
\end{aligned}
$$

- (the precedent sum with $\alpha$ and $\beta$ exchanged)

$$
\begin{aligned}
= & 2 g^{\gamma \beta}\left(F_{t^{\prime} t^{\gamma}}^{(1)} c_{\nu}^{\alpha \mu} t_{X}^{\nu}+F_{t_{X}^{\prime}}^{(1)} c_{\nu \sigma}^{\alpha \mu} t_{X}^{\nu} t_{X}^{\sigma}\right)-\frac{1}{12} u_{j} \gamma_{i j}\left(\frac{1}{\sigma_{j}}+\frac{\sigma_{j}}{\sigma_{i}^{2}}\right) \frac{\psi_{i}^{\alpha} \psi_{j}^{\beta} \psi_{i \nu}}{\psi_{i 1} \psi_{j 1}} t_{X X}^{\nu} \\
& +\frac{1}{24} \partial_{X}\left(2 u_{j} \gamma_{i j} \frac{\sigma_{i}}{\sigma_{j}} \frac{\psi_{i}^{\alpha} \psi_{j}^{\beta}}{\psi_{i 1} \psi_{j 1}}+\left(u_{i}+u_{j}\right) \gamma_{i j} \frac{\psi_{i}^{\alpha} \psi_{j}^{\beta}}{\psi_{i 1}^{2}}\right) \\
& +\left(\partial_{X} g^{\gamma \beta}\right)\left(F_{t^{\mu}}^{(1)} t_{X}^{\gamma} c_{\nu}^{\alpha \mu} t_{X}^{\nu}+F_{t_{X}^{\mu}}^{(1)} t_{X}^{\gamma} c_{\nu \sigma}^{\alpha \mu} t_{X}^{\nu} t_{X}^{\sigma}\right) \\
& +g^{\gamma \beta} \partial_{X}\left(F_{t_{X}^{\mu}}^{(1)} c_{\gamma \nu}^{\alpha \mu} t_{X}^{\nu}+3 F_{t_{X}^{\mu}}^{(1)} c_{\gamma}^{\alpha \mu} t_{X}^{\nu}+3 F_{t_{X}^{\mu} t_{X}^{\nu}}^{(1)} c_{\gamma}^{\alpha \mu} t_{X X}^{\nu}-F_{t_{X}^{\mu} t_{X}^{\gamma} c_{\nu}^{\alpha \mu} t_{X X}^{\nu}}\right. \\
& \left.-F_{t^{\mu}}^{(1)} c_{\gamma}^{\alpha \mu}\right)+2 g^{\gamma \beta}\left(F_{t^{\mu}}^{(1)} c_{\gamma \nu}^{\alpha \mu} t_{X}^{\nu}+F_{t_{X}^{\mu}}^{(1)} c_{\nu \rho \gamma}^{\alpha \mu} t_{X}^{\nu} t_{X}^{\rho}+F_{t_{X}^{\mu}}^{(1)} c_{\nu \gamma}^{\alpha \mu} t_{X X}^{\nu}\right) \\
& +3 \Gamma_{\mu}^{\gamma \beta} \frac{\partial w^{\alpha}}{\partial t_{X X}^{\gamma}} t_{X}^{\mu}+\Gamma_{\mu}^{\beta \gamma} \frac{\partial w^{\alpha}}{\partial t_{X X}^{\gamma}} t_{X}^{\mu}+3 \Gamma_{\mu}^{\beta \gamma} \partial_{X}\left(\frac{\partial w^{\alpha}}{\partial t_{X X X}^{\gamma}}\right) t_{X}^{\mu} \\
& +3 \frac{\partial^{2} g^{\gamma \beta}}{\partial X^{2}} \frac{\partial w^{\alpha}}{\partial t_{X X X}^{\gamma}}+3 \frac{\partial\left(\Gamma_{\mu}^{\gamma \beta} t_{X}^{\mu}\right)}{\partial X} \frac{\partial w^{\alpha}}{\partial t_{X X X}^{\gamma}} \\
& -(\text { the precedent sum with } \alpha \text { and } \beta \text { exchanged })
\end{aligned}
$$

$$
\begin{aligned}
= & 2 g^{\gamma \beta}\left(F_{t^{\mu} t^{\gamma}}^{(1)} c_{\nu}^{\alpha \mu} t_{X}^{\nu}+F_{t_{X}^{\mu}}^{(1)} c_{\nu \sigma}^{\alpha \mu} t_{X}^{\nu} t_{X}^{\sigma}\right) \\
& +\frac{1}{24} t_{X}^{\sigma} \frac{\partial}{\partial t^{\sigma}}\left(2 u_{j} \gamma_{i j} \frac{\sigma_{i}}{\sigma_{j}} \frac{\psi_{i}^{\alpha} \psi_{j}^{\beta}}{\psi_{i 1} \psi_{j 1}}+\left(u_{i}+u_{j}\right) \gamma_{i j} \frac{\psi_{i}^{\alpha} \psi_{j}^{\beta}}{\psi_{i 1}^{2}}\right) \\
& -2 q_{\gamma}\left(F_{t_{X}^{\mu} t_{X}^{\gamma}}^{(1)} c_{\nu \sigma}^{\alpha \mu} c_{\rho}^{\beta \gamma} t_{X}^{\rho} t_{X}^{\nu} t_{X}^{\sigma}+F_{t^{\mu} t_{X}^{\gamma}}^{(1)} c_{\sigma}^{\alpha \mu} c_{\rho}^{\beta \gamma} t_{X}^{\rho} t_{X}^{\sigma}\right)
\end{aligned}
$$




$$
\begin{aligned}
& +\left\{\left(3(1+d)-4 q_{\beta}\right)\left(F_{t_{X}^{\mu}}^{(1)} t_{X}^{\gamma} c_{\nu \sigma}^{\alpha \mu} c_{\rho}^{\beta \gamma} t_{X}^{\rho} t_{X}^{\nu} t_{X}^{\sigma}+F_{t^{\mu}}^{(1)} t_{X}^{\gamma} c_{\sigma}^{\alpha \mu} c_{\rho}^{\beta \gamma} t_{X}^{\rho} t_{X}^{\sigma}\right)\right. \\
& +q_{\beta} F_{t_{X}^{\mu}}^{(1)} t^{\nu} c_{\gamma}^{\alpha \mu} c_{\sigma}^{\beta \gamma} t_{X}^{\sigma} t_{X}^{\nu}-3 q_{\beta} F_{t_{X}^{\mu}}^{(1)} c_{\gamma \nu}^{\alpha \mu} c_{\sigma}^{\beta \gamma} t_{X}^{\sigma} t_{X}^{\nu}-3 q_{\beta} F_{t_{X}^{\mu}}^{(1)} c_{\gamma}^{\alpha \mu} c_{\sigma \nu}^{\beta \gamma} t_{X}^{\sigma} t_{X}^{\nu} \\
& \left.-2 q_{\beta} F_{t^{\mu}}^{(1)} c_{\gamma}^{\alpha \mu} c_{\sigma}^{\gamma \beta} t_{X}^{\sigma}\right\}- \text { (the precedent sum with } \alpha \text { and } \beta \text { exchanged) } \\
& =\frac{1}{24}\left\{\left(u_{j}-u_{k}\right) \gamma_{i k} \gamma_{k j} \sigma_{k} \frac{\psi_{i}^{\alpha} \psi_{j}^{\beta}}{\psi_{i 1}^{2} \psi_{k 1}}+3\left(u_{l}-u_{j}\right) \gamma_{i j} \gamma_{j l} \sigma_{i} \frac{\psi_{i}^{\alpha} \psi_{l}^{\beta}}{\psi_{i 1} \psi_{j 1}^{2}}\right. \\
& +2\left(u_{i}-u_{j}\right) \gamma_{i j} \gamma_{i l} \sigma_{i} \frac{\psi_{i}^{\alpha} \psi_{j}^{\beta} \psi_{l 1}}{\psi_{i 1}^{4}}-\left(u_{i}+u_{j}\right) \gamma_{i j} \gamma_{i l} \sigma_{i} \frac{\psi_{j}^{\beta} \psi_{l}^{\alpha}}{\psi_{i 1}^{3}} \\
& +\left(u_{j}-u_{k}\right) \gamma_{i k} \gamma_{k j} \sigma_{i} \frac{\psi_{i}^{\alpha} \psi_{j}^{\beta}}{\psi_{i 1}^{3}}+2\left(u_{j}-u_{l}\right) \gamma_{i j} \gamma_{j l} \frac{\sigma_{j}^{2}}{\sigma_{i}} \frac{\psi_{j}^{\alpha} \psi_{l}^{\beta}}{\psi_{j 1}^{3}} \\
& \left.+d \gamma_{i k} \sigma_{k} \frac{\psi_{i}^{\alpha} \psi_{k}^{\beta}}{\psi_{i 1}^{2} \psi_{k 1}}+2 d \gamma_{i j} \sigma_{i} \frac{\psi_{i}^{\alpha} \psi_{j}^{\beta}}{\psi_{i 1} \psi_{j 1}^{2}}-d \gamma_{i j} \sigma_{i} \frac{\psi_{j}^{\alpha} \psi_{i}^{\beta}}{\psi_{i 1}^{3}}\right\} \\
& +\frac{\gamma_{i j}}{24}\left\{3 q_{\beta} \sigma_{i} \frac{\psi_{i}^{\alpha} \psi_{j}^{\beta}}{\psi_{i 1}^{3}}+\left(q_{\beta}-3(1+d)\right)\left(\sigma_{j} \frac{\psi_{i}^{\alpha} \psi_{j}^{\beta}}{\psi_{j 1} \psi_{i 1}^{2}}+\sigma_{i} \frac{\psi_{i}^{\alpha} \psi_{j}^{\beta}}{\psi_{i 1}^{3}}\right)\right. \\
& +\left(5 q_{\beta}-6(1+d)\right) \sigma_{j} \frac{\psi_{j}^{\alpha} \psi_{i}^{\beta}}{\psi_{j 1} \psi_{i 1}^{2}}-4 q_{\beta} \sigma_{i} \frac{\psi_{i}^{\alpha} \psi_{i}^{\beta} \psi_{j 1}}{\psi_{i 1}^{4}}-2 q_{\beta} \sigma_{j} \frac{\psi_{j}^{\alpha} \psi_{j}^{\beta}}{\psi_{i 1} \psi_{j 1}^{2}} \\
& \left.-2 q_{\beta} \frac{\sigma_{j}^{2}}{\sigma_{i}} \frac{\psi_{j}^{\alpha} \psi_{j}^{\beta}}{\psi_{j 1}^{3}}\right\}-(\text { the precedent sum with } \alpha \text { and } \beta \text { exchanged }) \\
& =\frac{1}{24}\left(\frac{d}{2}-q_{\beta}\right)\left(\gamma_{i j} \sigma_{j} \frac{\psi_{i}^{\alpha} \psi_{j}^{\beta}}{\psi_{i 1}^{2} \psi_{j 1}}+3 \gamma_{i j} \sigma_{i} \frac{\psi_{i}^{\alpha} \psi_{j}^{\beta}}{\psi_{i 1} \psi_{j 1}^{2}}-2 \gamma_{i j} \sigma_{i} \frac{\psi_{i}^{\alpha} \psi_{i}^{\beta} \psi_{j 1}}{\psi_{i 1}^{4}}\right. \\
& \left.+\gamma_{i j} \sigma_{i} \frac{\psi_{i}^{\alpha} \psi_{j}^{\beta}}{\psi_{i 1}^{3}}+\gamma_{i j} \sigma_{i} \frac{\psi_{i}^{\alpha} \psi_{j}^{\beta}}{\psi_{i 1}^{3}}-2 \gamma_{i j} \frac{\sigma_{j}^{2}}{\sigma_{i}} \frac{\psi_{j}^{\alpha} \psi_{j}^{\beta}}{\psi_{j 1}^{3}}\right) \\
& +\frac{d}{24}\left\{\gamma_{i k} \sigma_{k} \frac{\psi_{i}^{\alpha} \psi_{k}^{\beta}}{\psi_{i 1}^{2} \psi_{k 1}}+2 \gamma_{i j} \sigma_{i} \frac{\psi_{i}^{\alpha} \psi_{j}^{\beta}}{\psi_{i 1} \psi_{j 1}^{2}}-\gamma_{i j} \sigma_{i} \frac{\psi_{j}^{\alpha} \psi_{i}^{\beta}}{\psi_{i 1}^{3}}\right\} \\
& +\frac{\gamma_{i j}}{24}\left\{3 q_{\beta} \sigma_{i} \frac{\psi_{i}^{\alpha} \psi_{j}^{\beta}}{\psi_{i 1}^{3}}+\left(q_{\beta}-3(1+d)\right)\left(\sigma_{j} \frac{\psi_{i}^{\alpha} \psi_{j}^{\beta}}{\psi_{j 1} \psi_{i 1}^{2}}+\sigma_{i} \frac{\psi_{i}^{\alpha} \psi_{j}^{\beta}}{\psi_{i 1}^{3}}\right)\right. \\
& +\left(5 q_{\beta}-6(1+d)\right) \sigma_{j} \frac{\psi_{j}^{\alpha} \psi_{i}^{\beta}}{\psi_{j 1} \psi_{i 1}^{2}}-4 q_{\beta} \sigma_{i} \frac{\psi_{i}^{\alpha} \psi_{i}^{\beta} \psi_{j 1}}{\psi_{i 1}^{4}}-2 q_{\beta} \sigma_{j} \frac{\psi_{j}^{\alpha} \psi_{j}^{\beta}}{\psi_{i 1} \psi_{j 1}^{2}} \\
& \left.-2 q_{\beta} \frac{\sigma_{j}^{2}}{\sigma_{i}} \frac{\psi_{j}^{\alpha} \psi_{j}^{\beta}}{\psi_{j 1}^{3}}\right\}-(\text { the precedent sum with } \alpha \text { and } \beta \text { exchanged }) \\
& =\frac{1}{24}\left(2 q_{\beta}-d-3\right) \gamma_{i j} \sigma_{i}\left(\frac{\psi_{i}^{\alpha} \psi_{j}^{\beta}}{\psi_{i 1} \psi_{j 1}^{2}}+\frac{\psi_{i}^{\alpha} \psi_{j}^{\beta}}{\psi_{i 1}^{3}}-\frac{\psi_{i}^{\alpha} \psi_{i}^{\beta} \psi_{j 1}}{\psi_{i 1}^{4}}-\frac{\psi_{i}^{\alpha} \psi_{i}^{\beta}}{\psi_{i 1}^{2} \psi_{j 1}}\right) \\
& \text { - (the precedent sum with } \alpha \text { and } \beta \text { exchanged) }
\end{aligned}
$$




$$
=\frac{1}{24}\left(d+3-2 q_{\beta}\right) c_{\gamma}^{\alpha \nu} c_{\nu \mu}^{\beta \mu} t_{X}^{\gamma}-\frac{1}{24}\left(d+3-2 q_{\alpha}\right) c_{\gamma}^{\beta \nu} c_{\nu \mu}^{\alpha \mu} t_{X}^{\gamma}
$$

Lemma is proved.

Proof of Theorem 2 Let's denote

$$
\widetilde{H}^{\beta, 0}=\frac{1}{24} \int w_{\mu \nu}^{\beta} t_{X}^{\mu} t_{X}^{\nu} d X, \quad F_{\gamma}^{\beta}=\eta^{\beta \beta^{\prime}} \frac{\partial^{2} F}{\partial t^{\beta^{\prime}} \partial t^{\gamma}},
$$

where $w_{\mu \nu}^{\beta}$ are defined in (3.12), then from Lemma 3 we see that the equations in (3.8) with $p=0$ can be written as

$$
\begin{aligned}
\frac{\partial t^{\alpha}}{\partial T_{\beta, 0}} & =c_{\gamma}^{\alpha \beta}(t) t_{X}^{\gamma}+\varepsilon^{2} \partial_{X}\left(\eta^{\alpha \gamma} \frac{\delta \widetilde{H}^{\beta, 0}}{\delta t^{\gamma}}+\hat{h}^{\alpha \gamma} \partial_{X}^{2} F_{\gamma}^{\beta}+\frac{1}{2} \partial_{X} \hat{h}^{\alpha \gamma} \partial_{X} F_{\gamma}^{\beta}\right) \\
= & c_{\gamma}^{\alpha \beta}(t) t_{X}^{\gamma}+\varepsilon^{2}\left(\hat{b}_{\gamma \mu}^{\alpha \beta}(t) t_{X}^{\gamma} t_{X X}^{\mu}+\hat{a}_{\gamma \mu \nu}^{\alpha \beta}(t) t_{X}^{\gamma} t_{X}^{\mu} t_{X}^{\nu}+\right. \\
& \left.+\hat{p}_{\gamma}^{\alpha \beta}(t) t_{X X X}^{\gamma}\right)+\mathcal{O}\left(\varepsilon^{4}\right),
\end{aligned}
$$

where the coefficients $\hat{b}_{\gamma \mu}^{\alpha \beta}(t), \hat{a}_{\gamma \mu \nu}^{\alpha \beta}(t), \hat{p}_{\gamma}^{\alpha \beta}(t)$ have the expression

$$
\begin{aligned}
\hat{p}_{\gamma}^{\alpha \beta}= & \frac{1}{12} c_{\mu \nu}^{\alpha \beta} c_{\gamma}^{\mu \nu}, \\
\hat{a}_{\gamma \mu \nu}^{\alpha \beta}= & \frac{1}{72}\left(\eta ^ { \alpha \sigma } \left(\partial_{\sigma} \partial_{\nu} w_{\gamma \mu}^{\beta}+\partial_{\sigma} \partial_{\gamma} w_{\mu \nu}^{\beta}\right.\right. \\
& \left.+\partial_{\sigma} \partial_{\mu} w_{\gamma \nu}^{\beta}-2 \partial_{\mu} \partial_{\nu} w_{\gamma \sigma}^{\beta}-2 \partial_{\mu} \partial_{\gamma} w_{\nu \sigma}^{\beta}-2 \partial_{\nu} \partial_{\gamma} w_{\mu \sigma}^{\beta}\right) \\
& +\eta^{\xi \zeta}\left(6 c_{\xi \zeta}^{\alpha \sigma} c_{\sigma \gamma \mu \nu}^{\beta}+3 c_{\xi \zeta \gamma}^{\alpha \sigma} c_{\sigma \mu \nu}^{\beta}+3 c_{\xi \zeta \mu}^{\alpha \sigma} c_{\sigma \gamma \nu}^{\beta}\right. \\
& \left.\left.+3 c_{\xi \zeta \nu}^{\alpha \sigma} c_{\sigma \gamma \mu}^{\beta}+c_{\xi \zeta \gamma \mu}^{\alpha \sigma} c_{\sigma \nu}^{\beta}+c_{\xi \zeta \gamma \nu}^{\alpha \sigma} c_{\sigma \mu}^{\beta}+c_{\xi \zeta \mu \nu}^{\alpha \sigma} c_{\sigma \gamma}^{\beta}\right)\right) \\
\hat{b}_{\gamma \mu}^{\alpha \beta}= & \frac{1}{12}\left(\eta^{\alpha \sigma}\left(-2 \partial_{\gamma} w_{\mu \sigma}^{\beta}+\partial_{\sigma} w_{\gamma \mu}^{\beta}-\partial_{\mu} w_{\gamma \sigma}^{\beta}\right)\right. \\
& \left.+\eta^{\xi \zeta}\left(3 c_{\xi \zeta}^{\alpha \sigma} c_{\sigma \gamma \mu}^{\beta}+\frac{3}{2} c_{\xi \zeta \gamma}^{\alpha \sigma} c_{\sigma \mu}^{\beta}+\frac{1}{2} c_{\xi \zeta \mu}^{\alpha \sigma} c_{\sigma \gamma}^{\beta}\right)\right)
\end{aligned}
$$

with $w_{\beta \gamma}^{\alpha}$ defined by (3.12), and $\hat{h}^{\alpha \beta}$ are defined in (5.4). On the other hand, from the bihamiltinian relation (2.82) we have

$$
\left(\frac{1+d}{2}-q_{\beta}\right) \frac{\partial t^{\alpha}}{\partial T_{\beta, 0}}=\left\{t^{\alpha}, \int t^{\beta}(X) d X\right\}_{2}^{\prime}+\mathcal{O}\left(\varepsilon^{4}\right),
$$

which together with (5.30) leads to the expression for the coefficients $p_{\gamma}^{\alpha \beta}(t), a_{\gamma \mu \nu}^{\alpha \beta}(t), b_{\gamma \mu}^{\alpha \beta}(t)$ in the formula (3.14)

$$
\begin{aligned}
& p_{\gamma}^{\alpha \beta}(t)=\left(\frac{1}{2}-\mu_{\beta}\right) \hat{p}_{\gamma}^{\alpha \beta}(t), \quad a_{\gamma \mu \nu}^{\alpha \beta}(t)=\left(\frac{1}{2}-\mu_{\beta}\right) \hat{a}_{\gamma \mu \nu}^{\alpha \beta}, \\
& b_{\gamma \mu}^{\alpha \beta}(t)=\left(\frac{1}{2}-\mu_{\beta}\right) \hat{b}_{\gamma \mu}^{\alpha \beta}(t) .
\end{aligned}
$$


The expression (3.15) of the coefficients $h^{\alpha \beta}$ follows from Lemma 5, formulas (3.19)(3.21) are obtained by using Lemma 6 and the anti-symmetry condition of the second Poisson bracket. In fact, if we denote $\tilde{r}^{\alpha \beta}, \tilde{f}^{\alpha \beta}$ and $\tilde{p}^{\alpha \beta}$ the coefficients before $\varepsilon^{2} \delta^{\prime \prime}(X-Y), \varepsilon^{2} \delta^{\prime}(X-Y)$ and $\varepsilon^{2} \delta(X-Y)$ in the second Poisson bracket $\left\{t^{\alpha}(X), t^{\beta}(Y)\right\}_{2}$ respectively, then the antisymmetry condition of the second Poisson bracket gives us

$$
\begin{aligned}
& \tilde{r}^{\alpha \beta}+\tilde{r}^{\beta \alpha}=3 \partial_{X} h^{\alpha \beta} \\
& \partial_{X} \tilde{f}^{\alpha \beta}+\partial_{X}^{3} h^{\alpha \beta}-\partial_{X}^{2} \tilde{r}^{\alpha \beta}=\tilde{p}^{\alpha \beta}+\tilde{p}^{\beta \alpha} .
\end{aligned}
$$

Formula (3.19) follows immediately from (5.35) and Lemma 6. From (5.30) it follows that

$$
\tilde{p}^{\alpha \beta}=\left(\frac{1+d}{2}-q_{\beta}\right) \partial_{X}\left(\eta^{\alpha \gamma} \frac{\delta \widetilde{H}^{\beta, 0}}{\delta t^{\gamma}}+h^{\alpha \gamma} \partial_{X}^{2} F_{\gamma}^{\beta}+\frac{1}{2} \partial_{X} h^{\alpha \gamma} \partial_{X} F_{\gamma}^{\beta}\right)
$$

So from (5.36) and the above expression of $\tilde{p}^{\alpha \beta}$ we obtain

$$
\begin{aligned}
\tilde{f}^{\alpha \beta} & =-\partial_{X}^{2} h^{\alpha \beta}+\partial_{X} \tilde{r}^{\alpha \beta} \\
& +\left(\frac{1+d}{2}-q_{\beta}\right)\left(\eta^{\alpha \gamma} \frac{\delta \widetilde{H}^{\beta, 0}}{\delta t^{\gamma}}+h^{\alpha \gamma} \partial_{X}^{2} F_{\gamma}^{\beta}+\frac{1}{2} \partial_{X} h^{\alpha \gamma} \partial_{X} F_{\gamma}^{\beta}\right) .
\end{aligned}
$$

which leads to formula $(3.20)$ and (3.21). We have thus verified the formula (3.14). The remaining part of the theorem follows from (2.82). Theorem is proved.

Proof of Proposition 3 For the correction of the expression of the first Possion bracket, let's replace the functions $w^{\alpha}$ by $\frac{\partial^{2} G}{\partial X \partial T_{\alpha, 0}}$ in the identity (5.1], then a direct calculation aided by the anti-symmetry condition of the Poisson bracket gives the expression for $\tilde{a}^{\alpha \beta}, \tilde{b}^{\alpha \beta}, \tilde{e}^{\alpha \beta}$. For the correction of the expression of the second Poisson bracket, from the identity (5.14) with $w^{\alpha}$ replaced by $\frac{\partial^{2} G}{\partial X \partial T_{\alpha, 0}}$ we can easily get the expression for the coefficients $a^{\alpha \beta}$ and $b^{\alpha \beta}$, however, it's not easy to get the simplified expression for the coefficients $q^{\alpha \beta}$ and $e^{\alpha \beta}$ in this way. We use instead the relation

$$
\left(\frac{1-d}{2}+q_{\beta}\right)\left\{v^{\alpha}(X), H_{\beta, 0}\right\}_{1}=\left\{v^{\alpha}(X), H_{\beta,-1}\right\}_{2}
$$

with $H_{\beta,-1}=\int v_{\beta}(X) d X, H_{\beta, 0}=\int \frac{\partial F(v(X))}{\partial v^{\beta}(X)} d X$ and the infinitesimal Bäcklund transform

$$
t^{\alpha}=v^{\alpha}+\varepsilon^{2} \frac{\partial^{2} G}{\partial X \partial T_{\alpha, 0}},
$$

to get the expression for the coefficient $q^{\alpha \beta}$, then by using the anti-symmetry condition of the Poisson bracket we get the expression for the coefficient $e^{\alpha \beta}$. Proposition is proved. 


\section{Genus one Gromov-Witten invariants and G-function of a Frobenius manifold}

In the paper [21] Getzler studied recursion relations for the genus one Gromov-Witten invariants of smooth projective varieties. He derived a remarkable system of linear differential equations for a generating function $G=G\left(t^{2}, \ldots, t^{n}\right)$ of these invariants. The system can be written in the following form:

$$
\begin{aligned}
& \sum_{1 \leq \alpha_{1}, \alpha_{2}, \alpha_{3}, \alpha_{4} \leq n} z_{\alpha_{1}} z_{\alpha_{2}} z_{\alpha_{3}} z_{\alpha_{4}}\left(3 c_{\alpha_{1} \alpha_{2}}^{\mu} c_{\alpha_{3} \alpha_{4}}^{\nu} \frac{\partial^{2} G}{\partial t^{\mu} \partial t^{\nu}}-4 c_{\alpha_{1} \alpha_{2}}^{\mu} c_{\alpha_{3} \mu}^{\nu} \frac{\partial^{2} G}{\partial t^{\alpha_{4}} \partial t^{\nu}}\right. \\
& -c_{\alpha_{1} \alpha_{2}}^{\mu} c_{\alpha_{3} \alpha_{4} \mu}^{\nu} \frac{\partial G}{\partial t^{\nu}}+2 c_{\alpha_{1} \alpha_{2} \alpha_{3}}^{\mu} c_{\alpha_{4} \mu}^{\nu} \frac{\partial G}{\partial t^{\nu}}+\frac{1}{6} c_{\alpha_{1} \alpha_{2} \alpha_{3}}^{\mu} c_{\alpha_{4} \mu \nu}^{\nu} \\
& \left.+\frac{1}{24} c_{\alpha_{1} \alpha_{2} \alpha_{3} \alpha_{4}}^{\mu} c_{\mu \nu}^{\nu}-\frac{1}{4} c_{\alpha_{1} \alpha_{2} \nu}^{\mu} c_{\alpha_{3} \alpha_{4} \mu}^{\nu}\right)=0
\end{aligned}
$$

The l.h.s. must be equal to zero identically in $z_{1}, \ldots, z_{n}$. The notations for the coefficients $c_{\beta \delta}^{\alpha}, c^{\alpha \beta \gamma \delta}$ are defined in (3.13). Now we solve this system for an arbitrary semisimple Frobenius manifold.

Proof of Theorem 3. Let us rewrite the system (6.1) in the canonical coordinates. At this end we first do a linear change of the indeterminates

$$
z_{\alpha} \mapsto w_{i}:=\sum_{\alpha=1}^{n} \frac{\psi_{i \alpha}}{\psi_{i 1}} z_{\alpha} .
$$

Instead of the partial derivatives of $G(t)$ and of $F(t)$ we substitute in (6.1) the corresponding covariant derivatives. For example,

$$
\begin{gathered}
\frac{\partial^{2} G}{\partial t^{\lambda} \partial t^{\mu}} \rightarrow \nabla_{i} \nabla_{j} G \\
c_{\alpha \beta \gamma}^{\delta} \rightarrow \nabla_{i} \nabla_{j} \nabla_{k} \nabla^{l} F
\end{gathered}
$$

etc. Here $\nabla$ is the Levi-Civita flat connection for the metric $<$, $>$ written in the curvilinear coordinates $u_{i}$. Recall that the metric becomes diagonal in the canonical coordinates

$$
<,>=\sum_{i=1}^{n} \psi_{i 1}^{2} d u_{i}{ }^{2} .
$$

The only nontrivial Christoffel coefficients of the connection are

$$
\begin{gathered}
\Gamma_{i j}^{i}=\gamma_{i j} \frac{\psi_{j 1}}{\psi_{i 1}}, \Gamma_{i i}^{j}=-\gamma_{i j} \frac{\psi_{i 1}}{\psi_{j 1}}, i \neq j \\
\Gamma_{i i}^{i}=-\sum_{k \neq i} \gamma_{i k} \frac{\psi_{k 1}}{\psi_{i 1}} .
\end{gathered}
$$


From the definition of the canonical coordinates we have

$$
\nabla_{i} \nabla_{j} \nabla^{k} F=\delta_{i}^{k} \delta_{j}^{k} .
$$

This simplifies the computation. Finally we obtain for the polynomial (6.1) in $w_{1}, \ldots, w_{n}$ the following structure

1). The coefficient in front of $w_{i}^{4}$ is equal to

$$
-\frac{\partial^{2} G}{\partial u^{i^{2}}}+P_{i i} .
$$

2). The coefficient in front of $w_{i}^{3} w_{j}$ for $i<j$ is equal to

$$
-4 \frac{\partial^{2} G}{\partial u^{i} \partial u^{j}}+4 P_{i j} .
$$

$3)$. The coefficient in front of $w_{i}^{2} w_{j}^{2}$ for $i<j$ is equal to

$$
6 \frac{\partial^{2} G}{\partial u^{i} \partial u^{j}}-6 P_{i j} .
$$

4). All other coefficients of the polynomial (6.1) vanish. Here $P_{i j}=P_{j i}$ is a complicated expression in $u_{1}, \ldots, u_{n}, \psi_{11}, \ldots, \psi_{n 1}, \gamma_{12}, \ldots, \gamma_{n-1 n}$.

From the above structure of the coefficients we immediately derive the uniqueness part of Theorem 4. Indeed, the general solution of the corresponding linear homogeneous system

$$
\frac{\partial^{2} G}{\partial u^{i} \partial u^{j}}=0
$$

is

$$
G=\sum_{i} c_{i} u_{i}+c_{0}
$$

for arbitrary constant coefficients. The quasihomogeneity equation (3.29) in the canonical coordinates reads

$$
\sum_{j=1}^{n} u^{j} \frac{\partial G}{\partial u^{j}}=\gamma
$$

Hence $c_{1}=\ldots=c_{n}=0$ and $G=$ const.

To find the first derivatives of $G$ we differentiate (6.7) w.r.t. $u_{i}$. This gives

$$
\frac{\partial G}{\partial u^{i}}=-\sum_{j} u^{j} \frac{\partial^{2} G}{\partial u^{i} \partial u^{j}}, i=1, \ldots, n .
$$

So

$$
\frac{\partial G}{\partial u^{i}}=-\sum_{j} u^{j} P_{i j} .
$$


After tedious calculations we obtain the following formula

$$
\begin{aligned}
24 \frac{\partial G}{\partial u^{i}}= & \sum_{j} \frac{\gamma_{i j}^{2}\left(u_{j}-u_{i}\right)\left[\psi_{i 1}^{4}-10 \psi_{i 1}^{2} \psi_{j 1}^{2}+\psi_{j 1}^{4}\right]}{\psi_{i 1}^{2} \psi_{j 1}^{2}} \\
& +\sum_{j} \gamma_{i j} \frac{\left(\psi_{i 1}^{2}+\psi_{j 1}^{2}\right)}{\psi_{i 1} \psi_{j 1}}\left[\frac{1}{\psi_{i 1}} \sum_{k \neq j} V_{i k} \psi_{k 1}-\frac{1}{\psi_{j 1}} \sum_{k \neq i} V_{j k} \psi_{k 1}\right] \\
& +\sum_{j} \gamma_{i j}\left(\frac{\psi_{j 1}}{\psi_{i 1}}-\frac{\psi_{i 1}}{\psi_{j 1}}\right)
\end{aligned}
$$

where, we recall, $V_{i j}=\left(u_{j}-u_{i}\right) \gamma_{i j}$. Using that $\psi_{i 1}$ is an eigenvector of $V$ we rewrite the formula in the following way

$$
\frac{\partial G}{\partial u^{i}}=\frac{1}{2} \sum_{j \neq i} \frac{V_{i j}^{2}}{u_{i}-u_{j}}-\frac{1}{24} \sum_{k \neq i} \gamma_{i k}\left(\frac{\psi_{i 1}}{\psi_{k 1}}-\frac{\psi_{k 1}}{\psi_{i 1}}\right)
$$

Using (2.19) and (4.9) we recognize in the r.h.s. the derivative

$$
\frac{\partial}{\partial u^{i}}\left[\log \tau_{I}-\frac{1}{24} \log \left(\psi_{11} \ldots \psi_{n 1}\right)\right]
$$

It remains to observe that

$$
\operatorname{det} \frac{\partial t^{\alpha}}{\partial u^{i}}=\psi_{11 \ldots} \ldots \psi_{n 1}
$$

up to an inessential constant. One can easily check that

$$
\sum_{i} \frac{\partial G}{\partial u^{i}}=0
$$

SO

$$
\frac{\partial G}{\partial t^{1}}=0
$$

The formula (3.30) is proved.

Let us derive the formula (3.32) for the constant $\gamma$. We have

$$
\sum_{i=1}^{n} u^{i} \partial_{i} \log \tau_{I}=\frac{1}{2} \sum_{j \neq i} \frac{u_{i} V_{i j}^{2}}{u_{i}-u_{j}}=\frac{1}{2} \sum_{i<j} V_{i j}^{2}=-\frac{1}{4} \operatorname{Trace} V^{2}=-\frac{1}{4} \sum_{\alpha=1}^{n} \mu_{\alpha}^{2} .
$$

The second term in the formula for $G$ gives

$$
\sum_{i=1}^{n} u_{i} \partial_{i} \log \left(\psi_{11} \ldots \psi_{n 1}\right)=\sum_{j=1}^{n} \psi_{j 1}^{-1} \sum_{i=1}^{n} u_{i} \partial_{i} \psi_{j 1}
$$

But

$$
\psi_{j 1}=\sqrt{\frac{\partial t^{n}}{\partial u^{j}}}
$$


and

$$
\begin{gathered}
\sum_{i=1}^{n} u_{i} \partial_{i} t^{n}=(1-d) t^{n} \text { for } d \neq 1 \\
\sum_{i=1}^{n} u_{i} \partial_{i} t^{n}=r_{n} \text { for } d=1
\end{gathered}
$$

(the Euler identity). So

$$
\sum_{i=1}^{n} u^{i} \partial_{i} \psi_{j 1}=-\frac{d}{2} \psi_{j 1}
$$

This proves the formula (3.32). Theorem is proved.

Definition. The function (3.30) is called G-function of the Frobenius manifold.

We begin our examples with the case $n=2$. In the 2-dimensional case, we write the free energy $F$ in the form

$$
F=\frac{1}{2}\left(t^{1}\right)^{2} t^{2}+f\left(t^{2}\right)
$$

The Getzler's equations (6.1) are reduced to

$$
48 f^{(3)} \frac{\partial^{2} G}{\partial t^{2} \partial t^{2}}-24 f^{(4)} \frac{\partial G}{\partial t^{2}}-f^{(5)}=0
$$

(cf. [25]). For the free energy

$$
F=\frac{1}{2}\left(t^{1}\right)^{2} t^{2}+c\left(t^{2}\right)^{h+1}
$$

where $c$ is an arbitrary non-zero constant, the $G$-function is

$$
G=-\frac{1}{24} \frac{(2-h)(3-h)}{h} \log \left(t^{2}\right)
$$

Particularly, the $G$-function vanishes for the $A_{2}$ topological minimal model (the case $h=3)$. The constant $\gamma$ equals

$$
\gamma=\frac{d(1-3 d)}{24}
$$

since $d=1-\frac{2}{h}$. For the free energy of the $C P^{1}$ model

$$
F=\frac{1}{2}\left(t^{1}\right)^{2} t^{2}+e^{t^{2}}
$$

the $G$-function reads

$$
G=-\frac{1}{24} t^{2} .
$$

The constant is

$$
\gamma=-\frac{1}{12}
$$


Observe that the $G$-function is analytic everywhere on the Frobenius manifold only for $d=\frac{1}{3}$ (the $A_{2}$ topological minimal model) and for $d=1$, i.e., for the $C P^{1}$ topological sigma model.

Let us consider now examples with $n=3$. We will take the list of examples of Frobenius manifolds with good analytic properties from [11].

1) For the polynomial free energy related to $A_{3}$,

$$
F=\frac{1}{2}\left(t^{1}\right)^{2} t^{3}+\frac{1}{2} t^{1}\left(t^{2}\right)^{2}+\frac{1}{4}\left(t^{2}\right)^{2}\left(t^{3}\right)^{2}+\frac{1}{60}\left(t^{3}\right)^{5}
$$

we have $G=0, \gamma=0$;

2) For the polynomial free energy related to $B_{3}$,

$$
F=\frac{1}{2}\left(t^{1}\right)^{2} t^{3}+\frac{1}{2} t^{1}\left(t^{2}\right)^{2}+\frac{1}{6}\left(t^{2}\right)^{3} t^{3}+\frac{1}{6}\left(t^{2}\right)^{2}\left(t^{3}\right)^{3}+\frac{1}{210}\left(t^{3}\right)^{7},
$$

we have

$$
G=-\frac{1}{48} \log \left(2 t^{2}-3\left(t^{3}\right)^{2}\right), \quad \gamma=-\frac{1}{72} .
$$

3) For the polynomial free energy related to the symmetry group of icosahedron,

$$
F=\frac{1}{2}\left(t^{1}\right)^{2} t^{3}+\frac{1}{2} t^{1}\left(t^{2}\right)^{2}+\frac{1}{6}\left(t^{2}\right)^{3}\left(t^{3}\right)^{2}+\frac{1}{20}\left(t^{2}\right)^{2}\left(t^{3}\right)^{5}+\frac{1}{3960}\left(t^{3}\right)^{11},
$$

we have

$$
G=-\frac{1}{20} \log \left(t^{2}-\left(t^{3}\right)^{3}\right), \quad \gamma=-\frac{3}{100} .
$$

4) For the free energy of the $C P^{2}$ model,

$$
F=\frac{1}{2}\left(t^{1}\right)^{2} t^{3}+\frac{1}{2} t^{1}\left(t^{2}\right)^{2}+\sum_{k \geq 1} N_{k}^{(0)} \frac{\left(t^{3}\right)^{3 k-1}}{(3 k-1) !} e^{k t^{2}}
$$

where $N_{k}^{(0)}$ are the number of rational curves of degree $k$ on $C P^{2}$ which meet $3 k-1$ generic points, for example, $N_{1}^{(0)}=N_{2}^{(0)}=1, N_{3}^{(0)}=12, N_{4}^{(0)}=620$. The $G$-function has the form

$$
G=-\frac{t^{2}}{8}+\sum_{k \geq 1} N_{k}^{(1)} \frac{\left(t^{3}\right)^{3 k}}{(3 k) !} e^{k t^{2}},\left.\quad \frac{\partial G}{\partial t^{2}}\right|_{t^{2}=z, t^{3}=1}=\frac{\phi^{\prime \prime \prime}-27}{8\left(27+2 \phi^{\prime}-3 \phi^{\prime \prime}\right)}, \quad \gamma=-\frac{3}{8},
$$

where $\phi$ is defined by (1.21), and $N_{k}^{(1)}$ are the number of elliptic plane curves of degree $k$ which meet $3 k$ generic points, for example, $N_{1}^{(1)}=N_{2}^{(1)}=0, N_{3}^{(1)}=1, N_{4}^{(1)}=225$.

5) For the free energy

$$
F=\frac{1}{2}\left(t^{1}\right)^{2} t^{3}+\frac{1}{2} t^{1}\left(t^{2}\right)^{2}+\left(t^{2}\right)^{4} \phi\left(t^{3}-2 r \log \left(t^{2}\right)\right)
$$


(here $d=1, r>0$ ) we obtain a solution of WDVV with good analytic properties only for $r=\frac{3}{2}, 1$ or $\frac{1}{2}$ [11]. These solutions correspond to extended affine Weyl groups of type $\tilde{A}_{2}, \tilde{C}_{2}, \tilde{G}_{2}$ respectively [13]. For all of them $\gamma=-1 / 16$. Particularly, for $\tilde{A}_{2}$

$$
\phi(z)=-\frac{1}{24}+e^{z}
$$

then $G=-\frac{1}{24} t^{3}$,

For $\tilde{C}_{2}$

$$
\phi(z)=-\frac{1}{48}+a e^{z}+\frac{a^{2}}{2} e^{2 z}
$$

where $a$ is an arbitrary non-zero constant, the $G$-function is

$$
G=-\frac{1}{24} t^{3}-\frac{1}{48} \log \left(16 a e^{t^{3}}-\left(t^{2}\right)^{2}\right) .
$$

Finally, for $\tilde{G}_{2}$

$$
\phi(z)=-\frac{1}{72}+\frac{2}{3} e^{z}+\frac{3}{2} e^{2 z}+\frac{9}{16} e^{4 z},
$$

the $G$-function is

$$
G=-\frac{1}{24} t^{3}-\frac{1}{12} \log \left(12 e^{t^{3}}-t^{2}\right) .
$$

6) We now take the free energy

$$
F=\frac{1}{2}\left(t^{1}\right)^{2} t^{3}+\frac{1}{2} t^{1}\left(t^{2}\right)^{2}-\frac{1}{16}\left(t^{2}\right)^{4} \phi\left(t^{3}\right),
$$

where $\phi(z)$ satisfies the Chazy equation

$$
\phi^{\prime \prime \prime}=6 \phi \phi^{\prime \prime}-9\left(\phi^{\prime}\right)^{2},
$$

(here $d=1, r=r_{3}=0$ ). Then the $G$ function can be obtained from the equations

$$
\frac{\partial G}{\partial t^{2}}=-\frac{1}{8 t^{2}}, \quad \frac{\partial G}{\partial t^{3}}=-\frac{1}{4} \phi\left(t^{3}\right), \quad \gamma=-\frac{1}{16} .
$$

Particularly, for the case

$$
\phi\left(t^{3}\right)=8 \pi i E_{2}\left(t^{3}\right)=4 \frac{d}{d t^{3}} \log \eta\left(t^{3}\right)
$$

where $\eta(\tau)$ is the Dedekind function, $E_{2}(\tau)$ is the second Eisenstein series (see [11]) we obtain

$$
G=-\log \left[\eta\left(t^{3}\right)\left(t^{2}\right)^{\frac{1}{8}}\right] .
$$

We see that, for $n=3$, only on the Frobenius manifold (6.11) (the free energy of the $A_{3}$ topological minimal model), and on the Frobenius manifold (6.15) related to 
the extended affine Weyl group $\tilde{A}_{2}$ the $G$-function are manifestly analytic everywhere. For the $C P^{2}$ sigma model the $G$-function is regular on the open subset where

$$
27+2 \phi^{\prime}-3 \phi^{\prime \prime} \neq 0
$$

From equations of associativity for the function (6.12) it can be seen (see [4]) that in the points $x_{0}$ where

$$
3 \phi^{\prime \prime}\left(x_{0}\right)-2 \phi^{\prime}\left(x_{0}\right)-27=0
$$

the series (6.13) diverges. Analytic properties of the $G$-function $(\sqrt[6.13)]{ })$ deserve a separate investigation.

Remark. In the cases $B_{3}, H_{3}, \tilde{B}_{2}, \tilde{G}_{2}$ the $G$-function has logarithmic branching on the part of the nilpotent locus of the Frobenius manifold, where $u_{i}=u_{j}$ for some $i \neq j$. The coefficients of our hierarchy will have singularities in these points. Probably, appearance of these singularities suggests not to select these Frobenius manifolds for a construction of a physically consistent model of 2D TFT.

\section{$7 \quad$ Some examples}

We now compare the dispersion expansions of some well known examples of bi-Hamiltonian integrable systems with those given in Theorem 1 and Theorem 2.

Example 1 Let's start with the detailed consideration of the simplest example of $\mathrm{KdV}$ hierarchy. We take the Lax operator in the form

$$
L=\frac{1}{2}\left(\varepsilon \partial_{X}\right)^{2}+u(X)
$$

Then the two compatible Poisson brackets related to this operator is given by

$$
\begin{aligned}
& \{u(X), u(Y)\}_{1}=\delta^{\prime}(X-Y) \\
& \{u(X), u(Y)\}_{2}=u(X) \delta^{\prime}(X-Y)+\frac{1}{2} u_{X}(X) \delta(X-Y)+\frac{\varepsilon^{2}}{8} \delta^{\prime \prime \prime}(X-Y)
\end{aligned}
$$

(They are derived from the formulae (7.15) and (7.16) in Example 2). Starting from the Casimir

$$
H_{-1}=\int u(X) d X
$$

of the first Poisson bracket we can construct a hierarchy of commuting Hamiltonians $H_{p}$ by using the following recursion relation

$$
\left\{u(X), H_{p-1}\right\}_{2}=\left(\frac{1}{2}+p\right)\left\{u(X), H_{p}\right\}_{1},
$$

i.e.,

$$
\left(u(X) \partial_{X}+\frac{1}{2} u_{X}(X)+\frac{\varepsilon^{2}}{8} \partial_{X}^{3}\right) \frac{\delta H_{p-1}}{\delta u}=\left(\frac{1}{2}+p\right) \partial_{X} \frac{\delta H_{p}}{\delta u} .
$$


Note that the factor $\left(\frac{1}{2}+p\right)$ does not appear in the usual recursion relation for the $\mathrm{KdV}$ hierarchy, we use this factor here to meet the topological recursion relation of the $A_{1}$ topological minimal model. Let's list the first four Hamiltonians

$$
\begin{aligned}
& H_{-1}=\int u(X) d X, \quad H_{0}=\int \frac{1}{2} u(X)^{2} d X, \\
& H_{1}=\int\left(\frac{1}{6} u(X)^{3}-\frac{1}{24} \varepsilon^{2} u_{X}(X)^{2}\right) d X, \\
& H_{2}=\int\left(\frac{1}{24} u(X)^{4}-\frac{1}{24} \varepsilon^{2} u(X) u_{X}(X)^{2}+\frac{1}{480} \varepsilon^{4} u_{X X}(X)^{2}\right) d X .
\end{aligned}
$$

The KdV hierarchy is then given recursively as

$$
\begin{aligned}
\frac{\partial u}{\partial T^{0}} & =u_{X} \\
\frac{\partial u}{\partial T^{1}} & =u u_{X}+\frac{1}{12} \varepsilon^{2} u_{X X X} \\
\frac{\partial u}{\partial T^{p}} & =\left(\frac{1}{2}+p\right)^{-1}\left(\frac{1}{2} u_{X} \partial_{X}^{-1}+u+\frac{1}{8} \varepsilon^{2} \partial_{X}^{2}\right) \frac{\partial u}{\partial T^{p-1}} .
\end{aligned}
$$

Let's note that each flow of the KdV hierarchy can be written as a polynomial in $\varepsilon^{2}$. The parameter $\varepsilon$ can be introduced to the usual KdV hierarchy through the rescaling $X \mapsto \varepsilon X, T^{p} \mapsto \varepsilon T^{p}$. We write down explicitly the $\varepsilon^{0}$ and $\varepsilon^{2}$ terms in the hierarchy

$$
\begin{aligned}
& \frac{\partial u}{\partial T^{0}}=u_{X} \\
& \frac{\partial u}{\partial T^{1}}{ }_{1}=u u_{X}+\frac{1}{12} \varepsilon^{2} u_{X X X} \\
& \frac{\partial u}{\partial T^{p}}=\left\{u(X), H_{p}^{(0)}\right\}_{1}+\varepsilon^{2}\left\{u(X), H_{p}^{(1)}\right\}_{1}+\mathcal{O}\left(\varepsilon^{4}\right) .
\end{aligned}
$$

where

$$
H_{p}^{(0)}=\int \frac{u(X)^{p+2}}{(p+2) !} d X
$$

and

$$
\begin{aligned}
& H_{-1}^{(1)}=H_{0}^{(1)}=0 \\
& H_{p}^{(1)}=\int\left(-\frac{1}{24}\right) \frac{u(X)^{p-1}}{(p-1) !} u_{X}(X)^{2} d X
\end{aligned}
$$

We now take the free energy to be

$$
F=\frac{1}{6}\left(t^{1}\right)^{3}
$$

in this case the $G$-function $G=0$. Plugging this free energy into Theorem 1 and Theorem 2, and identify $t^{1}, T^{p, 1}, H_{1, p}, \delta H_{1, p}^{\prime}$ with $u, T^{p}, H_{p}^{(0)}, H_{p}^{(1)}$ respectively, we obtain, modulo $\mathcal{O}\left(\varepsilon^{4}\right)$, the above described $\mathrm{KdV}$ hierarchy and it's bihamiltonian structure. 
Example 2 More generally, let's consider the differential operators

$$
L=(\varepsilon \partial)^{N+1}+u_{N}(X)(\varepsilon \partial)^{N-1}+\ldots+u_{1}(X),
$$

where $\partial=\frac{\partial}{\partial X}$. For any pseudo-differential operator $Z$ of the form

$$
Z=(\varepsilon \partial)^{-1} Z_{1}+(\varepsilon \partial)^{-2} Z_{2}+\ldots+(\varepsilon \partial)^{-N} Z_{N},
$$

define the following two Hamiltonian mappings [2]:

$$
H_{1}: Z \mapsto[Z, L]_{+}, \quad H_{2}: Z \mapsto L(Z L)_{+}-(L Z)_{+} L+\frac{1}{N+1}\left[L, \int^{X} \operatorname{Res}[Z, L] d X\right],
$$

and the corresponding Poisson brackets

$$
\{\tilde{f}, \tilde{g}\}_{i}=\int \operatorname{Res}\left(H_{i}\left(\frac{\delta f}{\delta L}\right) \frac{\delta g}{\delta L}\right) d x, \quad i=1,2,
$$

for the functionals

$$
\tilde{f}=\int f d x, \quad \tilde{g}=\int g d x
$$

where

$$
\frac{\delta f}{\delta L}=\sum_{i=1}^{N}(\varepsilon \partial)^{-i} \frac{\delta f}{\delta u_{i}}, \quad \frac{\delta g}{\delta L}=\sum_{i=1}^{N}(\varepsilon \partial)^{-i} \frac{\delta g}{\delta u_{i}} .
$$

In the case of $N=2$, if we define

$$
t^{1}=u_{1}-\frac{1}{2} \varepsilon u_{2}^{\prime}, \quad t^{2}=\frac{1}{3} u_{2},
$$

then the first Poisson bracket is given as follows:

$$
\begin{aligned}
& \left\{t^{1}(X), t^{1}(Y)\right\}_{1}=0, \quad\left\{t^{1}(X), t^{2}(Y)\right\}_{1}=\delta^{\prime}(X-Y), \\
& \left\{t^{2}(X), t^{2}(Y)\right\}_{1}=0
\end{aligned}
$$

and the second Poisson bracket is given as

$$
\begin{aligned}
\left\{t^{1}(X), t^{1}(Y)\right\}_{2}= & -6\left(t^{2}\right)(X)^{2} \delta^{\prime}(X-Y)-6 t^{2}(X)\left(t^{2}\right)^{\prime}(X) \delta(X-Y) \\
& -\varepsilon^{2}\left(\frac{15}{4}\left(t^{2}\right)^{\prime}(X) \delta^{\prime \prime}(X-Y)+\frac{9}{4}\left(t^{2}\right)^{\prime \prime}(X) \delta^{\prime}(X-Y)\right. \\
& \left.+\frac{5}{2} t^{2}(X) \delta^{(3)}(X-Y)+\frac{1}{2}\left(t^{1}\right)^{(3)}(X) \delta(X-Y)\right)-\frac{\varepsilon^{4}}{6} \delta^{(5)}(X-Y), \\
\left\{t^{1}(X), t^{2}(Y)\right\}_{2}= & t^{1}(X) \delta^{\prime}(X-Y)+\frac{1}{3}\left(t^{1}\right)^{\prime}(X) \delta(X-Y), \\
\left\{t^{2}(X), t^{2}(Y)\right\}_{2}= & \frac{2}{3} t^{2}(X) \delta^{\prime}(X-Y)+\frac{1}{3}\left(t^{2}\right)^{\prime}(X) \delta(X-Y) \\
& +\frac{2 \varepsilon^{2}}{9} \delta^{(3)}(X-Y) .
\end{aligned}
$$


The integrable hierarchy has the form

$$
\frac{\partial t^{\alpha}}{\partial T^{\beta, p}}=\left\{t^{\alpha}(X), H_{\beta, p}\right\}_{1}
$$

where the Hamiltonians $H_{\beta, p}$ are recursively defined by

$$
\left\{t^{\alpha}(X), H_{\beta, p-1}\right\}_{2}=\left(\frac{1-d}{2}+p+q_{\beta}\right)\left\{t^{\alpha}(X), H_{\beta, p}\right\}_{1}
$$

with $H_{\beta,-1}=\int t_{\beta}(X) d X$.

Up to the $\varepsilon^{2}$ terms, the above Poisson brackets and the integrable hierarchy coincide with the Poisson brackets and the integrable hierarchy given in Theorem 1 and Theorem 2 with the free energy defined by

$$
F=\frac{1}{2}\left(t^{1}\right)^{2} t^{2}-\frac{3}{8}\left(t^{2}\right)^{4}
$$

and with the $G$-function $G=0$. This is the primary free energy of the $A_{2}$ topological minimal model of [0].

In the case of $N=3$, if we define

$$
t^{1}=u_{1}-\frac{1}{8} u_{3}^{2}-\frac{\varepsilon}{2} u_{2}^{\prime}+\frac{\varepsilon^{2}}{12} u_{3}^{\prime \prime}, \quad t^{2}=u_{2}-\varepsilon u_{3}^{\prime}, \quad t^{3}=u_{3},
$$

then the Poisson brackets defined by $(\overline{7.15}),(7.16)$ and the integrable system given in the form of $(\sqrt{7.22})$ and $(7.23)$ coincide, modulo $\varepsilon^{4}$, with the Poisson brackets and the integrable system given in Theorem 1 and Theorem 2 with the free energy defined by

$$
F=\frac{1}{8} t^{1}\left(t^{2}\right)^{2}+\frac{1}{8}\left(t^{1}\right)^{2} t^{3}-\frac{1}{64}\left(t^{2}\right)^{2}\left(t^{3}\right)^{2}+\frac{1}{3840}\left(t^{3}\right)^{5},
$$

and with the $G$-function $G=0$. This is the primary free energy of the $A_{3}$ topological minimal model $[i b i d]$. Formulae in (7.25) coincide with formulae in (4.38) of [6].

Example 3 The explicit bihamiltonian structure related to the Lie algebra of type $B_{2}$ is given, for example, in [1] in the following form:

$$
\begin{aligned}
\left\{u_{1}(X), u_{1}(Y)\right\}_{1} & =2 u_{2}(X) \delta^{\prime}(X-Y)+u_{2}^{\prime}(X) \delta(X-Y)-\varepsilon^{2} \delta^{(3)}(X-Y), \\
\left\{u_{1}(X), u_{2}(Y)\right\}_{1} & =2 \delta^{\prime}(X-Y) \\
\left\{u_{2}(X), u_{1}(Y)\right\}_{1} & =2 \delta^{\prime}(X-Y) \\
\left\{u_{2}(X), u_{2}(Y)\right\}_{1} & =0 \\
\left\{u_{1}(X), u_{1}(Y)\right\}_{2}= & 2 u_{2}(X) u_{1}(X) \delta^{\prime}(X-Y)+u_{2}^{\prime}(X) u_{1}(X) \delta(X-Y) \\
& +u_{2}(X) u_{1}^{\prime}(X) \delta(X-Y)-\varepsilon^{2}\left[\frac{3}{2} u_{2}^{\prime}(X)^{2} \delta^{\prime}(X-Y)\right. \\
& +6 u_{2}(X) u_{2}^{\prime}(X) \delta^{\prime \prime}(X-Y)+\frac{3}{2} u_{1}^{\prime}(X) \delta^{\prime \prime}(X-Y)
\end{aligned}
$$




$$
\begin{aligned}
& +4 u_{2}(X) u_{2}^{\prime \prime}(X) \delta^{\prime}(X-Y)+\frac{1}{2} u_{2}^{\prime}(X) u_{2}^{\prime \prime}(X) \delta(X-Y) \\
& +\frac{3}{2} u_{1}^{\prime \prime}(X) \delta^{\prime}(X-Y)+2 u_{2}(X)^{2} \delta^{(3)}(X-Y) \\
& +u_{1}(X) \delta^{(3)}(X-Y)+u_{2}(X) u_{2}^{(3)}(X) \delta(X-Y) \\
& \left.+\frac{1}{2} u_{1}^{(3)}(X) \delta(X-Y)\right]+\varepsilon^{4}\left[8 u_{2}^{\prime \prime}(X) \delta^{(3)}(X-Y)\right. \\
& +7 u_{2}^{(3)}(X) \delta^{\prime \prime}(X-Y)+5 u_{2}^{\prime}(X) \delta^{(4)}(X-Y) \\
& +3 u_{2}^{(4)}(X) \delta^{\prime}(X-Y)+2 u_{2}(X) \delta^{(5)}(X-Y) \\
& \left.+\frac{1}{2} u_{2}^{(5)}(X) \delta(X-Y)\right]-\frac{1}{2} \varepsilon^{6} \delta^{(7)}(X-Y), \\
\left\{u_{1}(X), u_{2}(Y)\right\}_{2}= & 2 u_{1}(X) \delta^{\prime}(X-Y)+\frac{1}{2} u_{1}^{\prime}(X) \delta(X-Y) \\
& -\varepsilon^{2}\left(u_{2}^{\prime}(X) \delta^{\prime \prime}(X-Y)+2 u_{2}(X) \delta^{(3)}(X-Y)\right)+\varepsilon^{4} \delta^{(5)}(X-Y), \\
\left\{u_{2}(X), u_{1}(Y)\right\}_{2}= & 2 u_{1}(X) \delta^{\prime}(X-Y)+\frac{3}{2} u_{1}^{\prime}(X) \delta(X-Y)-\varepsilon^{2}\left[u_{2}^{(3)}(X) \delta(X-Y)\right. \\
& \left.+5 u_{2}^{\prime}(X) \delta^{\prime \prime}(X-Y)+2 u_{2}(X) \delta^{(3)}(X-Y)++4 u_{2}^{\prime \prime}(X) \delta^{\prime}(X-Y)\right] \\
& +\varepsilon^{4} \delta^{(5)}(X-Y), \\
\left\{u_{2}(X), u_{2}(Y)\right\}_{2}= & u_{2}(X) \delta^{\prime}(X-Y)+\frac{1}{2} u_{2}^{\prime}(X) \delta(X-Y)-\frac{5}{2} \varepsilon^{2} \delta^{(3)}(X-Y), \quad(7.28)
\end{aligned}
$$

here we note that the above coordinates $u_{1}, u_{2}$ should be the coordinates $u_{1}, u_{0}$ respectively in [四], and there is a sign difference between the above first Poisson bracket and that of [四].

We now compare the above Poisson brackets with the Poisson brackets given by Theorem 1 and Theorem 2 with the free energy related to $B_{2}$. For this, let

$$
F=\frac{1}{2}\left(t^{1}\right)^{2} t^{2}+\frac{1}{15}\left(t^{2}\right)^{5}
$$

then the $G$-function is given by $G=-\frac{1}{48} \log \left(t^{2}\right)$, and the first and second Poisson brackets of Theorem 1 and Theorem 2 are given by

$$
\begin{gathered}
\left\{t^{1}(X), t^{1}(Y)\right\}_{1}=\left\{t^{2}(X), t^{2}(Y)\right\}_{1}=\mathcal{O}\left(\varepsilon^{4}\right), \\
\left\{t^{1}(X), t^{2}(Y)\right\}_{1}=\delta^{\prime}(X-Y)+\mathcal{O}\left(\varepsilon^{4}\right) ; \\
\left\{t^{1}(X), t^{1}(Y)\right\}_{2}=2 t^{2}(X)^{3} \delta^{\prime}(X-Y)+3 t^{2}(X)^{2}\left(t^{2}\right)^{\prime}(X) \delta(X-Y) \\
+\varepsilon^{2}\left(\frac{\left(t^{1}\right)^{\prime}(X)^{2} \delta^{\prime}(X-Y)}{32 t^{2}(X)^{2}}-\frac{\left(t^{1}\right)^{\prime}(X)^{2}\left(t^{2}\right)^{\prime}(X) \delta(X-Y)}{32 t^{2}(X)^{3}}\right. \\
+\frac{29\left(t^{2}\right)^{\prime}(X)^{2} \delta^{\prime}(X-Y)}{24}+\frac{13 t^{2}(X)\left(t^{2}\right)^{\prime}(X) \delta^{\prime \prime}(X-Y)}{4} \\
+\frac{\left(t^{1}\right)^{\prime}(X)\left(t^{1}\right)^{\prime \prime}(X) \delta(X-Y)}{32 t^{2}(X)^{2}}+\frac{25 t^{2}(X)\left(t^{2}\right)^{\prime \prime}(X) \delta^{\prime}(X-Y)}{12}
\end{gathered}
$$




$$
\begin{aligned}
& +\frac{5\left(t^{2}\right)^{\prime}(X)\left(t^{2}\right)^{\prime \prime}(X) \delta(X-Y)}{8}+\frac{13 t^{2}(X)^{2} \delta^{(3)}(X-Y)}{12} \\
& \left.\quad+\frac{\delta(X-Y) t^{2}(X)\left(t^{2}\right)^{(3)}(X) \delta(X-Y)}{2}\right), \\
& \left\{t^{1}(X), t^{2}(Y)\right\}_{2}=t^{1}(X) \delta^{\prime}(X-Y)+\frac{\left(t^{1}\right)^{\prime}(X) \delta(X-Y)}{4} \\
& \quad+\varepsilon^{2}\left(\frac{-\left(\left(t^{1}\right)^{\prime}(X) \delta^{\prime \prime}(X-Y)\right)}{24 t^{2}(X)}+\frac{t^{1}(X)\left(t^{2}\right)^{\prime}(X) \delta^{\prime \prime}(X-Y)}{24 t^{2}(X)^{2}}\right. \\
& \left.\quad-\frac{t^{1}(X) \delta^{(3)}(X-Y)}{24 t^{2}(X)}\right), \\
& \left\{t^{2}(X), t^{2}(Y)\right\}_{2}=\frac{t^{2}(X) \delta^{\prime}(X-Y)}{2}+\frac{\left(t^{2}\right)^{\prime}(X) \delta(X-Y)}{4} \\
& \quad+\frac{3 \varepsilon^{2} \delta^{(3)}(X-Y)}{16} .
\end{aligned}
$$

Now if we relate the variables $u_{1}, u_{2}$ to the variables $t^{1}, t^{2}$ by the following relation:

$$
t^{1}=u_{1}-\frac{1}{4} u_{2}^{2}+\frac{\varepsilon^{2}}{4} u_{2}^{\prime \prime}, \quad t^{2}=\frac{1}{2} u_{2}
$$

then the above first Poisson brackets coincide, modulo $\varepsilon^{4}$, with the Poisson brackets given in (7.27). While for the second Poisson brackets, they coincide with the Poisson brackets given in (7.28) only up to $\varepsilon^{0}$, and starting from the $\varepsilon^{2}$ terms, the two second Poisson brackets no longer coincide. This result is in accordance with the result of [16], where it was shown, by imposing the commutativity of the flows, that the integrable system (the tree-level one) related to the free energy (7.29) can not be extended beyond $\varepsilon^{2}$ terms.

Remark. In this section, the free energies corresponding to the Lie algebras of the types $A_{2}, A_{3}, B_{2}$ are different from those given in Section 6 , they are related by a rescaling.

Example 4 Consider the Toda lattice equation with open boundary

$$
\begin{aligned}
\frac{\partial u_{n}}{\partial t} & =v_{n}-v_{n-1}, \\
\frac{\partial v_{n}}{\partial t} & =e^{u_{n+1}}-e^{u_{n}}, \quad n \in \mathbf{Z} .
\end{aligned}
$$

If we introduce the slow variables $T=t \varepsilon, X=n \varepsilon$, and the new dependent variables $\tilde{u}(X)=u_{n}, \tilde{v}(X)=v_{n}$, then the Toda lattice equations lead to

$$
\begin{aligned}
\frac{\partial \tilde{u}}{\partial T} & =\frac{1}{\varepsilon}(\tilde{v}(X)-\tilde{v}(X-\varepsilon)) \\
\frac{\partial \tilde{v}}{\partial T} & =\frac{1}{\varepsilon}\left(e^{\tilde{u}(X+\varepsilon)}-e^{\tilde{u}(X)}\right) .
\end{aligned}
$$


This system has the bi-Hamiltonian structure

$$
\begin{aligned}
& \frac{\partial \tilde{u}}{\partial T}=\left\{\tilde{u}(X), H_{0}\right\}_{1}=\left\{\tilde{u}(X), H_{-1}\right\}_{2}, \\
& \frac{\partial \tilde{v}}{\partial T}=\left\{\tilde{v}(X), H_{0}\right\}_{1}=\left\{\tilde{v}(X), H_{-1}\right\}_{2},
\end{aligned}
$$

where the Poisson brackets are defined by

$$
\begin{aligned}
& \{\tilde{u}(X), \tilde{u}(Y)\}_{1}=\{\tilde{v}(X), \tilde{v}(Y)\}_{1}=0 \\
& \{\tilde{u}(X), \tilde{v}(Y)\}_{1}=\frac{1}{\varepsilon}(\delta(X-Y)-\delta(X-Y-\varepsilon)) \\
& \{\tilde{u}(X), \tilde{u}(Y)\}_{2}=\frac{1}{\varepsilon}(\delta(X-Y+\varepsilon)-\delta(X-Y-\varepsilon)) \\
& \{\tilde{v}(X), \tilde{u}(Y)\}_{2}=\frac{1}{\varepsilon}(\delta(X-Y+\varepsilon)-\delta(X-Y)) \tilde{v}(X) \\
& \{\tilde{v}(X), \tilde{v}(Y)\}_{2}=\frac{1}{\varepsilon}\left(e^{\tilde{u}(X+\varepsilon)} \delta(X-Y+\varepsilon)-e^{\tilde{u}(X)} \delta(X-Y-\varepsilon)\right)
\end{aligned}
$$

and the Hamiltonians are given by

$$
H_{-1}=\int \tilde{v}(X) d X, \quad H_{0}=\int\left(\frac{1}{2} \tilde{v}(X)^{2}+e^{\tilde{u}(X)}\right) d X
$$

We construct the hierarchy of integrable systems

$$
\frac{\partial \tilde{u}}{\partial T^{p}}=\left\{\tilde{u}(X), H_{p}\right\}_{1}, \quad \frac{\partial \tilde{v}}{\partial T^{p}}=\left\{\tilde{v}(X), H_{p}\right\}_{1}
$$

with the Hamiltonians $H_{p}$ recursively defined by

$$
\left\{\tilde{u}(X), H_{p-1}\right\}_{2}=(p+1)\left\{\tilde{u}(X), H_{p}\right\}_{1}, \quad\left\{\tilde{v}(X), H_{p-1}\right\}_{2}=(p+1)\left\{\tilde{v}(X), H_{p}\right\}_{1} .
$$

We identify $T^{0}$ with $T$.

Let's define again the following new variables:

$$
\begin{aligned}
t^{1}(X) & =\tilde{v}(X)-\frac{\varepsilon^{2}}{24} \tilde{v}^{\prime \prime}(X)+\mathcal{O}\left(\varepsilon^{4}\right), \\
t^{2}(X) & =\tilde{u}(X)+\frac{\varepsilon}{2} \tilde{u}^{\prime}(X)+\frac{\varepsilon^{2}}{24} \tilde{u}^{\prime \prime}(X)-\frac{\varepsilon^{3}}{48} \tilde{u}^{\prime \prime \prime}(X)+\mathcal{O}\left(\varepsilon^{4}\right),
\end{aligned}
$$

and expand the above Poisson brackets in Taylor series in $\varepsilon$, we obtain

$$
\begin{aligned}
& \left\{t^{1}(X), t^{1}(Y)\right\}_{1}=\left\{t^{2}(X), t^{2}(Y)\right\}_{1}=0 \\
& \left\{t^{1}(X), t^{2}(Y)\right\}_{1}=\delta^{\prime}(X-Y)-\frac{\varepsilon^{2}}{12} \delta^{(3)}(X-Y)+\mathcal{O}\left(\varepsilon^{4}\right) \\
& \left\{t^{1}(X), t^{1}(Y)\right\}_{2}=2 e^{t^{2}(X)} \delta^{\prime}(X-Y)+e^{t^{2}(X)}\left(t^{2}(X)\right)^{\prime} \delta(X-Y) \\
& \quad+\varepsilon^{2}\left(\frac{1}{6} \delta^{(3)}(X-Y)+\frac{1}{4}\left(t^{2}(X)\right)^{\prime} \delta^{\prime \prime}(X-Y)+\frac{1}{12}\left(\left(t^{2}(X)\right)^{\prime}\right)^{2} \delta^{\prime}(X-Y)\right.
\end{aligned}
$$




$$
\begin{aligned}
& +\frac{1}{4}\left(t^{2}(X)\right)^{\prime \prime} \delta^{\prime}(X-Y)+\frac{1}{12}\left(t^{2}(X)\right)^{\prime}\left(t^{2}(X)\right)^{\prime \prime} \delta(X-Y) \\
& \left.+\frac{1}{12}\left(t^{2}(X)\right)^{(3)} \delta(X-Y)\right) e^{t^{2}(X)}+\mathcal{O}\left(\varepsilon^{4}\right), \\
& \left\{t^{1}(X), t^{2}(Y)\right\}_{2}=t^{1}(X) \delta^{\prime}(X-Y)-\varepsilon^{2}\left(\frac{1}{12} t^{1}(X) \delta^{(3)}(X-Y)\right. \\
& \left.\quad+\frac{1}{12}\left(t^{1}(X)\right)^{\prime} \delta^{\prime \prime}(X-Y)\right)+\mathcal{O}\left(\varepsilon^{4}\right), \\
& \left\{t^{2}(X), t^{2}(Y)\right\}_{2}=2 \delta^{\prime}(X-Y)+\mathcal{O}\left(\varepsilon^{4}\right) .
\end{aligned}
$$

We also expand the Hamiltonians (7.40) and the integrable system (7.39) in Taylor series in $\varepsilon$. The Hamiltonians $H_{-1}$ and $H_{0}$ have the form

$$
\begin{aligned}
H_{-1}= & \int t^{1}(X) d X+\mathcal{O}\left(\varepsilon^{4}\right) \\
H_{0}= & \int\left(\frac{1}{2}\left(t^{1}(X)\right)^{2}+e^{t^{2}(X)}\right) d X \\
& \quad-\frac{\varepsilon^{2}}{12} \int\left(\frac{1}{2}\left(t_{X}^{1}(X)\right)^{2}+e^{t^{2}(X)}\left(t_{X}^{2}(X)\right)^{2}\right) d X+\mathcal{O}\left(\varepsilon^{4}\right) .
\end{aligned}
$$

Now if we put the $C P^{1}$ free energy

$$
F=\frac{1}{2}\left(t^{1}\right)^{2} t^{2}+e^{t^{2}}
$$

into Theorem 1-Theorem 3, and with $G$-function $G=-\frac{1}{24} t^{2}$, we get the Poisson brackets which coincide with those given in (7.44) modulo $\varepsilon^{4}$, and the Hamiltonians $H_{2, p}$ we get also coincide with $H_{p}$ modulo $\varepsilon^{4}$. This suggests that the Toda lattice hierarchy is the appropriate hierarchy of integrable systems behind the $C P^{1}$ model, as it was suggested in [16] from the point of view of commuting flows.

\section{Discussion}

We formulate here the conjectural shape of the integrable hierarchy to be considered starting from a Frobenius manifold and of its bihamiltonian structure in the form of genus expansion. The hierarchy must have the form

$$
\frac{\partial t}{\partial T^{\alpha, p}}=K_{\alpha, p}^{(0)}\left(t, t_{X}\right)+\sum_{k \geq 1} \varepsilon^{2 k} K_{\alpha, p}^{(k)}\left(t, t_{X}, t_{X X}, \ldots\right)=\left\{t(X), H_{\alpha, p}\right\}_{1}
$$

where the Hamiltonians and the first Poisson bracket must have the expansions

$$
\begin{aligned}
& H_{\alpha, p}=H_{\alpha, p}^{(0)}+\sum_{k \geq 1} \varepsilon^{2 k} H_{\alpha, p}^{(k)}, \\
& \left\{t^{\alpha}(X), t^{\beta}(Y)\right\}_{1}=\left\{t^{\alpha}(X), t^{\beta}(Y)\right\}_{1}^{(0)}+\sum_{k \geq 1} \varepsilon^{2 k}\left\{t^{\alpha}(X), t^{\beta}(Y)\right\}_{1}^{(k)}
\end{aligned}
$$


where

$$
\begin{aligned}
& H_{\alpha, p}^{(k)}=\int P_{\alpha, p}^{(k)}\left(t ; t_{X}, t_{X X}, \ldots\right) d X \\
& \left\{t^{\alpha}(X), t^{\beta}(Y)\right\}_{1}^{(k)}=\left.\sum_{s=0}^{2 k+1} A_{k, s}^{\alpha, \beta}\left(t ; t_{X}, t_{X X}, \ldots\right)\right|_{t=t(X)} \delta^{(s)}(X-Y) .
\end{aligned}
$$

The densities $P_{\alpha, p}^{(k)}\left(t ; t_{X}, t_{X X}, \ldots\right)$ and the coefficients $A_{k, s}^{\alpha, \beta}\left(t ; t_{X}, t_{X X}, \ldots\right)$ of the Poisson bracket are quasihomogeneous polynomials in $t_{X}, t_{X X}, \ldots$ of the degrees

$$
\begin{aligned}
& \operatorname{deg} P_{\alpha, p}^{(k)}\left(t ; t_{X}, t_{X X}, \ldots\right)=2 k, \\
& \operatorname{deg} A_{k, s}^{\alpha, \beta}\left(t ; t_{X}, t_{X X}, \ldots\right)=2 k+1-s
\end{aligned}
$$

where we assign the degrees

$$
\operatorname{deg} \partial_{X}^{m} t=m
$$

for any $m=1,2, \ldots$. The coefficients $K_{\alpha, p}^{(k)}\left(t ; t_{X}, t_{X X}, \ldots\right)$ of the hierarchy are also polynomials in the same variables of the degree

$$
\operatorname{deg} K_{\alpha, p}^{(k)}\left(t ; t_{X}, t_{X X}, \ldots\right)=2 k+1, \quad k=0,1, \ldots
$$

All the Hamiltonians must commute.

Remark. The dispersion expansions of the known integrable hierarchies obtained by simultaneous rescaling $x \mapsto \varepsilon x, t \mapsto \varepsilon t$ for any time variable $t$ contain also odd powers of $\varepsilon$. However, doing an appropriate $\varepsilon$-dependent change of dependent variables we can reduce the hierarchy and their Poisson brackets to the form postulated in this section. (See examples above in Section 7).

We expect that the quasihomogeneity (2.3) will not be involved in the construction of the hierarchy. If, however, it takes place then the coefficients of the first Poisson bracket must satisfy another quasihomogeneity condition. Let us introduce the extended Euler vector field

$$
\mathcal{E}:=E+\sum_{m \geq 1} \sum_{\alpha}\left(1-m-q_{\alpha}\right) \partial_{X}^{m} t^{\alpha} \frac{\partial}{\partial\left(\partial_{X}^{m} t^{\alpha}\right)},
$$

where the Euler vector field $E$ has the form (2.10). Then the coefficients of the first Poisson bracket (8.3) must satisfy the quasihomogeneity conditions

$$
\mathcal{L}_{\mathcal{E}} A_{k, s}^{\alpha \beta}\left(t ; t_{X}, t_{X X}, \ldots\right)=\left(k(d-3)+d+s-1-q_{\alpha}-q_{\beta}\right) A_{k, s}^{\alpha \beta}\left(t ; t_{X}, t_{X X}, \ldots\right) .
$$

Moreover, there exists another Poisson bracket with the structure similar to (8.3), (8.5)

$$
\begin{aligned}
& \left\{t^{\alpha}(X), t^{\beta}(Y)\right\}_{2}=\left\{t^{\alpha}(X), t^{\beta}(Y)\right\}_{2}^{(0)}+\sum_{k \geq 1} \varepsilon^{2 k}\left\{t^{\alpha}(X), t^{\beta}(Y)\right\}_{2}^{(k)} \\
& \left\{t^{\alpha}(X), t^{\beta}(Y)\right\}_{2}^{(k)}=\left.\sum_{s=0}^{2 k+1} B_{k, s}^{\alpha, \beta}\left(t ; t_{X}, t_{X X}, \ldots\right)\right|_{t=t(X)} \delta^{(s)}(X-Y),
\end{aligned}
$$


where $B_{k, s}^{\alpha, \beta}\left(t ; t_{X}, t_{X X}, \ldots\right)$ are polynomials in $t_{X}, t_{X X}, \ldots$ of the same degree $2 k+1-s$ in the sense of (8.8). The quasihomogeneity conditions for the coefficients of the second Poisson bracket have the form

$$
\mathcal{L}_{\mathcal{E}} B_{k, s}^{\alpha \beta}\left(t ; t_{X}, t_{X X}, \ldots\right)=\left(k(d-3)+d+s-q_{\alpha}-q_{\beta}\right) B_{k, s}^{\alpha \beta}\left(t ; t_{X}, t_{X X}, \ldots\right) .
$$

The Poisson brackets $\{,\}_{1}$ and $\{,\}_{2}$ must be compatible, i.e., any linear combination of them with arbitrary constant coefficients must be again a Poisson bracket. Besides

$$
\frac{\partial}{\partial t^{1}} B_{k, s}^{\alpha \beta}=A_{k, s}^{\alpha \beta}, \quad \frac{\partial}{\partial t^{1}} A_{k, s}^{\alpha \beta}=0 .
$$

All the equations of the hierarchy (8.1) with the numbers $(\alpha, p)$ such that

$$
\frac{1}{2}+\mu_{\alpha}+p \neq 0
$$

are Hamiltonian flows also w.r.t. the second Poisson bracket.

Additional conjecture about the bihamiltonian structure (8.3), (8.12) is that, for $d \neq 1$

$$
\begin{array}{ll}
\left\{t^{n}(X), t^{n}(Y)\right\}_{1}^{(k)}=0 & \text { for } k>0, \\
\left\{t^{n}(X), t^{n}(Y)\right\}_{2}^{(k)}=0 & \text { for } k>1 .
\end{array}
$$

Here the invariant definition of the coordinate $t^{n}$ is $t^{n}:=\eta_{1 \varepsilon} t^{\varepsilon}$. This conjecture means that the Virasoro algebra with the central charge (3.35) found for $d \neq 1$ in Corollary 1 above does not get deformations coming from the genera $\geq 2$. In other words, our bihamiltonian structure is a classical $W$-algebra with the conformal dimensions (2.31) and the central charge (3.35).

We recall that a Frobenius manifold $M^{n}$ is said to have good analytic properties if the primary free energy $F(t)$ has the form

$$
F(t)=\text { cubic terms }+ \text { analytic perturbation }
$$

near some point $t_{0} \in M^{n}$. (See [11]). For example, the point $t_{0}$ is the origin in the topological minimal models and it is the point of classical limit in the topological sigmamodels. For Frobenius manifolds with good analytic properties we expect that all the coefficients of the polynomials $A_{k, s}^{\alpha \beta}\left(t ; t_{X}, t_{X X}, \ldots\right), \quad B_{k, s}^{\alpha \beta}\left(t ; t_{X}, t_{X X}, \ldots\right)$ are analytic in $t$ near the point $t_{0}$. For the case $t_{0}=0, d<1$, i.e., the charges satisfy

$$
0 \leq q_{\alpha} \leq d<1
$$

the analyticity implies finiteness of all of the expansions of the Poisson bracket. Indeed, from (8.5) and (8.11) we obtain that

$$
k(d-3)+d+s-1-q_{\alpha}-q_{\beta} \leq k(d-1)+d .
$$


This number is nonnegative only if

$$
k \leq \frac{d}{1-d} .
$$

But all the degrees of the variables $t^{\alpha}$ are $1-q_{\alpha}>0$. So all the terms $\{,\}_{1}^{(k)}$ must vanish for $k>\frac{d}{1-d}$. Similarly, the terms in the expansion of the second Poisson bracket must vanish for $k>\frac{1+d}{1-d}$. All the examples of $1+1$ integrable hierarchies labeled by A-D-E Dynkin graphs are of this type. All the coefficients of the genus expansions are polynomials.

Recall, that a polynomial Frobenius manifold can be constructed for an arbitrary finite Coxeter group [11]. For this case

$$
d=1-\frac{2}{h}, \quad q_{\alpha}=1-\frac{m_{\alpha}+1}{h}
$$

where $h$ is the Coxeter number and $m_{\alpha}$ are the exponents of the Coxeter group. However, the bihamiltonian hierarchy (8.1) can be constructed for only simply-laced Dynkin graphs. Indeed, our formula (3.35) for the central charge coincides with the formula [20]

$$
c \varepsilon^{2}=12 \varepsilon^{2} \rho^{2}
$$

of the central charge of the classical $W$-algebras with the same Dynkin diagram exactly for the simply-laced case! Here $\rho$ is one half of the sum of positive roots. Our $\varepsilon$ is equal to $i \alpha$ of [20]. Recall, that for the simply-laced Coxeter groups our polynomial Frobenius manifolds correspond to the topological minimal models [7]. The constant $\gamma$ in (3.29) equals 0 . So the $G$-function is identically equal to 0 for the A - D - E polynomial Frobenius manifolds.

For $d \geq 1$ the expansions probably are infinite. The Jacobi identity for the Poisson brackets, commutativity of the Hamiltonians etc. are understood as identities for the formal power series in $\varepsilon^{2}$. In the paper we have constructed the first terms of the expansions and showed that they are in agreement with the assumptions we formulate in this section.

To proceed to the next order $\mathcal{O}\left(\varepsilon^{4}\right)$ we are to compute the Poisson brackets

$$
\left\{H_{\alpha, p}^{(0)}, H_{\beta, q}^{(1)}\right\}^{(1)}+\left\{H_{\alpha, p}^{(1)}, H_{\beta, q}^{(0)}\right\}^{(1)}+\left\{H_{\alpha, p}^{(1)}, H_{\beta, q}^{(1)}\right\}^{(0)}:=Q_{\alpha, p ; \beta, q} .
$$

Then the corrections to the Hamiltonians and to the Poisson brackets are to be determined from the linear equations

$$
\left\{H_{\alpha, p}^{(0)}, H_{\beta, q}^{(2)}\right\}^{(0)}+\left\{H_{\alpha, p}^{(2)}, H_{\beta, q}^{(0)}\right\}^{(0)}+\left\{H_{\alpha, p}^{(0)}, H_{\beta, q}^{(0)}\right\}^{(2)}=-Q_{\alpha, p ; \beta, q} .
$$

We do not expect that the deformed hierarchy and the Poisson brackets can be constructed for an arbitrary Frobenius manifold (cf. [16]). However, solvability of the linear system (8.24) together with the bihamiltonian property could give a clue to the problem of selection of "physical" solutions of WDVV equations of associativity. We plan to investigate this solvability in subsequent publications. 
We do not discuss in this paper the relations between the one-loop deformations of the hierarchy and the Virasoro algebra of [18, 19]. This is to be done in a subsequent publication. Another interesting problem is a relation between the hierarchy we contsruct and the recursion relations of [28.

\section{References}

[1] P. Casati and M. Pedroni, Drinfeld-Sokolov reduction on a simple Lie algebra from the bihamiltonian point of view. Lett. Math. Phys. 25(1992), 89-101.

[2] L. Dickey, Soliton equations and Hamiltonian systems. World Scientic, 1991.

[3] P.Di Francesco, C. Itzykson, J.-B. Zuber, Classical $W$-algebras, Comm. Math. Phys. 140 (1991) 543-567.

[4] P. Di Francesco, C. Itzykson, Quantum intersection rings, hep-th/9412175.

[5] R. Dijkgraaf, E. Verlinde, H. Verlinde, Notes on topological string theory and 2D quantum gravity, IASSNS-HEP-90/80.

[6] R. Dijkgraaf, E. Witten, Mean field theory, topological field theory, and multimatrix models, Nucl. Phys. B342 (1990), 486-522.

[7] R. Dijkgraaf, E. Verlinde, H. Verlinde, Topological strings in $d<1$. Nucl. Phys. B352 (1991), 59.

[8] R. Dijkgraaf, Intersection Theory, Integrable Hierarchies and Topological Field Theory, Lectures given at the Cargese Summer School on 'New Symmetry Principles in Quantum Field Theory,' July 16-27, 1991, hep-th/9201003.

[9] B. Dubrovin, Integrable systems in topological field theory, Nucl. Phys. B379 (1992), 627-689.

[10] B. Dubrovin, Topological conformal field theory from the point of view of integrable systems, In: Integrable Quantum Field Theories, Edited by L.Bonora, G.Mussardo, A.Schwimmer, L.Girardello, and M.Martellini, Plenum Press, NATO ASI series B310 (1993), 283 - 302.

[11] B. Dubrovin, Geometry of 2D topological field theories, in: Integrable Systems and Quantum Groups, Montecalini, Terme, 1993. Editor: M.Francaviglia, S. Greco. Springer Lecture Notes in Math. 1620 (1996), 120-348.

[12] B. Dubrovin, Painlevé equations in 2D topological field theories. In: Painlevé Property, One Century Later, Cargèse, 1996, math.AG/9803107.

[13] B. Dubrovin, Y. Zhang, Extended affine Weyl groups and Frobenius manifolds, Compositio Math. 111 (1998), 167-219. 
[14] T. Eguchi, H. Kanno, Toda Lattice Hierarchy and the Topological Description of the c=1 String Theory, Phys. Lett. B331 (1994), 330-334.

[15] T. Eguchi and S.-K. Yang, The Topological $C P^{1}$ Model and the Large-N Matrix Integral, Mod. Phys. Lett. A9 (1994), 2893-2902.

[16] T. Eguchi, Y. Yamada and S.-K. Yang, On the Genus Expansion in the Topological String Theory, Rev. Math. Phys. 7 (1995) 279.

[17] T. Eguchi, K. Hori, C.S. Xiong, Gravitational Quantum Cohomology, Int. J. Mod. Phys. A12 (1997), 1743-1782.

[18] T. Eguchi, K. Hori, C.S. Xiong, Quantum Cohomology and Virasoro Algebra, Phys.Lett. B402 (1997), 71-80.

[19] T. Eguchi, M. Jinzenji, C.S. Xiong, Quantum Cohomology and Free Field Representation, hep-th/9709152.

[20] V.A. Fateev, S.L. Lukyanov, Additional symmetries and exactly-solvable models in two-dimensional conformal field theory, parts I, II, and III, Sov. Sci. Rev. A15 (1990) 1.

[21] E. Getzler, Intersection theory on $\bar{M}_{1,4}$ and elliptic Gromov-Witten invariants, alg-geom/9612004, to appear in J. Amer. Math. Soc..

[22] A. Givental, Elliptic Gromov-Witten invariants and the generalized mirror conjecture, math.AG/9803053.

[23] K. Hori, Constraints for topological strings in $d \geq 1$, Nucl. Phys. B439 (1995), 395.

[24] M. Jimbo, T. Miwa, Y. Mori, M. Sato, Physica 1D (1980) 80; M. Jimbo, T. Miwa, Physica 2D (1981) 407-448.

[25] A. Kabanov, T. Kimura, Intersection numbers and rank one cohomological field theories in genus one, alg-geom/9706003.

[26] M. Kontsevich, Intersection theory on the moduli space of curves and the matrix Airy function. Comm. Math. Phys. 147 (1992), 1-23.

[27] M. Kontsevich, Yu. Manin, Gromov-Witten classes, quantum cohomology and enumerative geometry, Comm. Math. Phys. 164 (1994), 525-562.

[28] M. Kontsevich, Yu. I. Manin, Relations between the correlators of the topological sigma-model coupled to gravity, alg-geom/9708024.

[29] F. Magri, A simple model of the integrable Hamiltonian systems, J. Math. Phys. 19(1978), 1156-1162. 
[30] T. Miwa, Painlevé property of monodromy preserving deformation equations and the analyticity of $\tau$-function, Publ. RIMS 17 (1981), 703-721.

[31] E. Witten, On the structure of the topological phase of two-dimensional gravity, Nucl. Phys. B340 (1990), 281-332.

[32] E. Witten, Two-dimensional gravity and intersection theory on moduli space, Surv. in Diff. Geom. 1 (1991), 243-310.

[33] E. Witten, On the Kontsevich model and other models of two-dimensional gravity, preprint IASSNS-HEP-91/24. 